\title{
Evolution of a Disintegrin and Metalloproteinase Gene Family in Vertebrates
}

Shashwati Bhattacharya

Follow this and additional works at: https://researchrepository.wvu.edu/etd

\section{Recommended Citation}

Bhattacharya, Shashwati, "Evolution of a Disintegrin and Metalloproteinase Gene Family in Vertebrates" (2015). Graduate Theses, Dissertations, and Problem Reports. 5205.

https://researchrepository.wvu.edu/etd/5205

This Thesis is protected by copyright and/or related rights. It has been brought to you by the The Research Repository @ WVU with permission from the rights-holder(s). You are free to use this Thesis in any way that is permitted by the copyright and related rights legislation that applies to your use. For other uses you must obtain permission from the rights-holder(s) directly, unless additional rights are indicated by a Creative Commons license in the record and/ or on the work itself. This Thesis has been accepted for inclusion in WVU Graduate Theses, Dissertations, and Problem Reports collection by an authorized administrator of The Research Repository @ WVU. For more information, please contact researchrepository@mail.wvu.edu. 
Types of Co-existing Chronic Physical Conditions and Newly-diagnosed Depression, its Treatment and Economic Outcomes among Medicaid Beneficiaries with Type 2 Diabetes

\author{
By \\ Rituparna Bhattacharya \\ Dissertation submitted \\ to the School of Pharmacy \\ at West Virginia University \\ in partial fulfillment of the requirements for the degree of \\ Doctor of Philosophy \\ in \\ Pharmaceutical and Pharmacological Sciences
}

\author{
Usha Sambamoorthi, Ph.D., Chair \\ Nilanjana Dwibedi, Ph.D \\ Ranjita Misra, PhD, CHES, FASHA \\ Virginia Scott, Ph.D \\ Amy B. Wachholtz, Ph.D., M.Div, MS \\ Department of Pharmaceutical Systems and Policy \\ Morgantown, West Virginia \\ 2014 \\ Coexisting, Chronic Illnesses, Multimorbidity, Comorbidity
}

Keywords: Diabetes, Depression, Antidepressants, Psychotherapy, expenditure, Multiple

Copyright 2015 Rituparna Bhattacharya 


\title{
ABSTRACT \\ Types of Co-existing Chronic Physical Conditions and Newly-diagnosed Depression, its Treatment and Economic Outcomes among Medicaid Beneficiaries with Type 2 Diabetes
}

\author{
Rituparna Bhattacharya, B. Pharm., M.S.
}

Diabetes is a widely prevalent metabolic condition. Adults with type 2 diabetes mellitus (T2DM) also have many coexisting chronic physical conditions. Coexisting chronic physical conditions among individuals with T2DM may be concordant (conditions that overlap with T2DM in their pathogenesis and management plans such as cardiovascular diseases) or discordant (conditions with unrelated pathogenesis or management plans such as musculoskeletal disorders) or dominant (conditions whose severity eclipses all other illness management plans such as metastatic cancer). There is documented evidence on the negative consequences of depression in adults with T2DM. However, there is only limited knowledge on how the types of coexisting chronic physical conditions (defined as concordant, discordant or dominant conditions) influence the risk for developing depression, subsequent depression treatment patterns and economic consequences of depression treatment, among adults with T2DM. Therefore the aims of this dissertation were to examine (1) the association of risk of newly-diagnosed depression with types of coexisting chronic physical conditions among adults with T2DM (2) the association between types of coexisting chronic physical conditions and depression treatment among adults with T2DM and newly-diagnosed depression was analyzed and (3) whether the relationship between depression treatment and total and T2DM-related healthcare care expenditures vary by types of coexisting chronic physical conditions among non-elderly adult Medicaid beneficiaries with T2DM and newly-diagnosed depression. A retrospective longitudinal cohort study design was used. Patient-level data were obtained from multi-year, multi-state Medicaid claims. Non-elderly (ages 18-64), fee-for-service Medicaid beneficiaries with T2DM who were depression free were followed for a period of 12 months to identify newly-diagnosed depression. The final study population consisted of 59,857 Medicaid beneficiaries of whom $\mathrm{N}=5,974$ had newly diagnosed depression. After controlling for other risk factors, those with dominant conditions were at $17 \%$ higher risk ( $\mathrm{p}=0.0006)$ and those with both concordant and discordant conditions were found to be at $30 \%$ higher risk $(\mathrm{p}<.0001)$ to develop newly-diagnosed depression as compared to those with concordant conditions only. Individuals with dominant conditions $(\mathrm{p}<0.05)$ were less likely to receive depression treatment with only antidepressants compared to those with discordant conditions only. Individuals with dominant conditions were more likely to receive depression treatment with only psychotherapy $(\mathrm{p}<.01)$ as compared to those with discordant conditions only. No statistically significant associations were observed between types of coexisting chronic physical conditions and receipt of adequate depression treatment. As compared to no depression treatment, treating depression reduced total healthcare expenditures. As compared to no depression treatment, treatment with only antidepressants was associated with $17 \%$ reduction in total healthcare expenditures, treatment with only psychotherapy was associated with $22 \%$ reduction in total healthcare expenditures and treatment with both antidepressants and psychotherapy was associated with $28 \%$ reductions in total healthcare expenditures. As compared to no depression treatment, treatment with both antidepressants and psychotherapy was associated with reductions in total healthcare expenditures among all types of coexisting chronic physical condition groups. In summary, these results indicate that among adults with T2DM, newly-diagnosed depression rates, its treatment and economic benefits vary by types of coexisting chronic physical conditions. 
Dedicated To

My Dad, Mr. Utpal Bhattacharya,

My Mom, Mrs. Rita Bhattacharya, \&

My sweetheart Dr. Sandipan Bhattacharjee 


\section{ACKNOWLEDGEMENTS}

I would like to express my deepest gratitude to my major advisor Dr. Usha Sambamoorthi, for her guidance, support and patience during the course of Doctoral training at West Virginia University (WVU). Working with her helped not only helped me to learn academic and research skills, but also enriched my experience of being a professional graduate student. Her efforts were instrumental in shaping up my successful research career at WVU. She has also been a great source of social support for me and has guided me during many professional and personal decisions.

I would also like to extend my sincere thanks to my committee members, Dr. Virginia Scott, Dr. Nilanjana Dwibedi, Dr. Ranjita Misra and Dr. Amy Wachholtz. Their critical inputs and valuable suggestions have helped me to improve on my thesis. I would specially like to thank our department chair Dr. Suresh Madhavan. Dr. Madhavan has been very encouraging and is a constant source of professional and personal support to me. I would also like to convey my heartiest thanks to the entire faculty and staff members at the WVU, School of Pharmacy for their constant help and support. I will also like to thank Ms. Angie Frame, my department's office administrator. She is always a smiling face who is ready to help in any way she can. I special thanks goes to my colleagues and fellow graduate students, time spent with each one of my friends in the department has been memorable.

Higher education is an intense commitment and successful completion of it would not have been possible without the support of my parents. My mom and dad have always been dedicated towards my education and have helped me in every-way possible. Words are not enough to express my gratitude towards them. So, thank you Mom and Dad for being there for me, every step of the way.

I consider myself very lucky to have found my soul-mate, my dearest husband Sandipan. Having someone who can share and understand, all my work related woes and help me through it, is truly a blessing. Hard times would have been harder and successes would not have tasted the same without Sandipan by my side. My in-laws have also been very supportive of my career decisions and I thank them too.

So, a big thanks to all of you. It's all your good wishes and blessings that have brought me this far. I will always love you and will try to be there for you at every opportunity I get to be of any help. 


\section{Table of Contents}

CHAPTER 1: Background and Significance

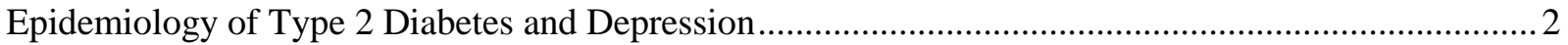

Coexisting chronic physical conditions, T2DM and Depression .......................................................... 3

Relationship between types of physical conditions and risk of depression ........................................ 3

Relationship between types of chronic physical conditions and treatment of depression ...................... 4

Economic benefits of treating depression and its association with types of chronic physical conditions 6

Need for the Study Using Non-Elderly Medicaid Population .......................................................... 6

Theoretical Framework Used to Categorize Types Of Coexisting Chronic Physical Conditions ............ 7

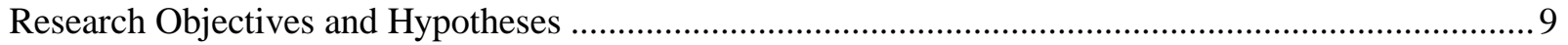

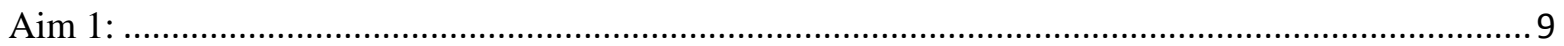

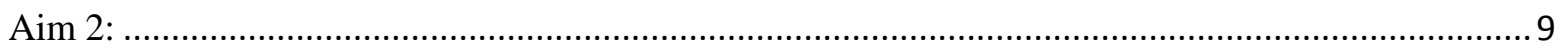

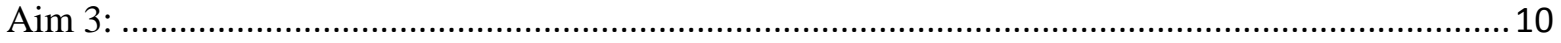

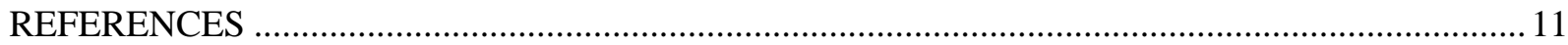

CHAPTER 2: Type of Coexisting Chronic Physical Conditions and the Risk of Newly-diagnosed Depression among Medicaid Beneficiaries with Type 2 Diabetes ....................................................... 14

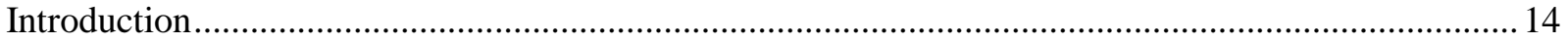

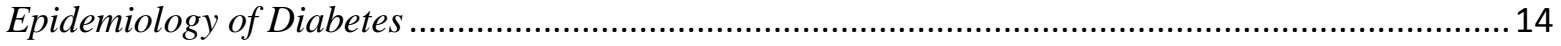

T2DM and Risk of Newly-diagnosed Depression .......................................................................... 14

Coexisting Chronic Physical Conditions among Adults withT2DM ........................................... 16

Coexisting Chronic Physical Conditions and Risk of Depression .................................................. 17

Coexisting Chronic Physical Conditions among Adults with T2DM and Risk of Depression............18

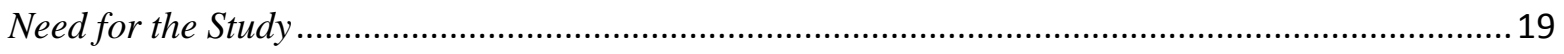

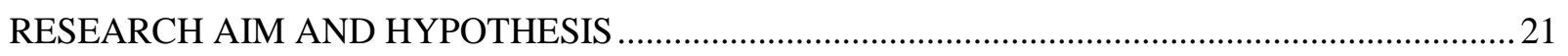

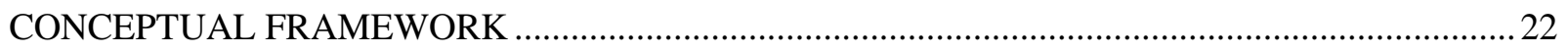

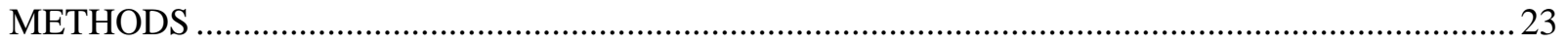

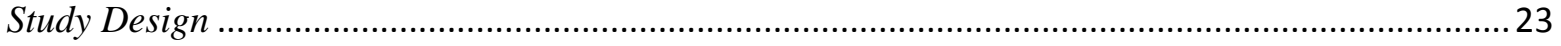

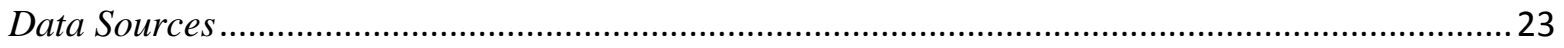

Identification of Study Cohort: Individuals with T2DM and Free of Depression at Baseline............ 24

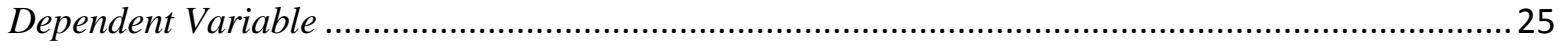

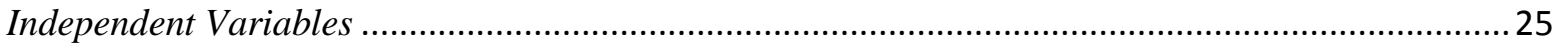

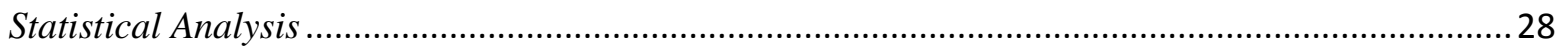

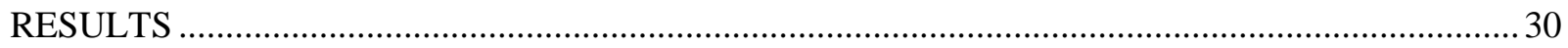




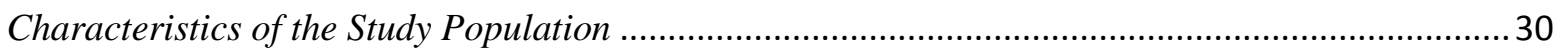

Study Population Characteristics by Types of Coexisting Chronic Physical Conditions ................... 30

Unadjusted Associations between Types of Coexisting Chronic Physical Conditions and Newly-

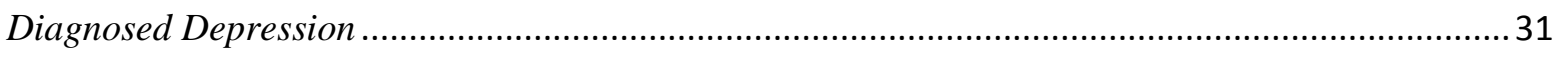

Relationships between Types of Coexisting Chronic Physical Conditions and Newly-Diagnosed

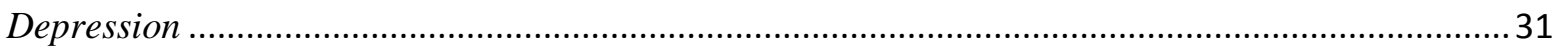

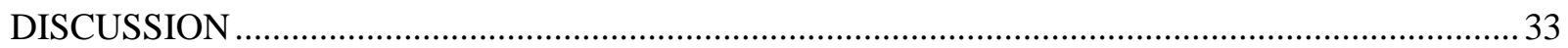

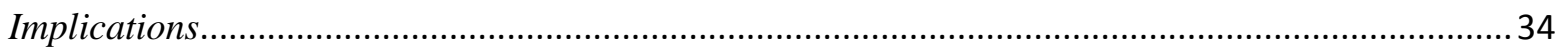

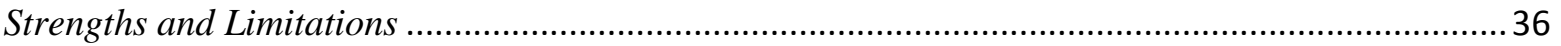

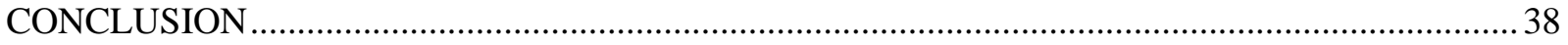

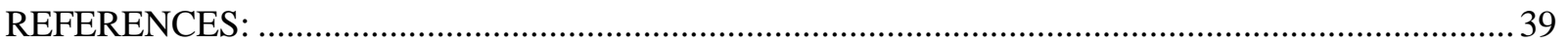

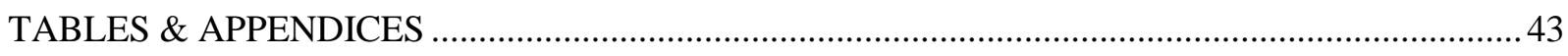

Table 1: Description of the Study Population by Types of Coexisting Chronic Physical Conditions among Medicaid Beneficiaries with Type 2 Diabetes Mellitus, Multi State Medicaid Claims - 2000 2008.

Table 2: Percentages of Newly-diagnosed Depression by Types of Coexisting Chronic Physical Conditions among Medicaid Beneficiaries with Type 2 Diabetes Mellitus, Multi-state Medicaid Claims data $-2000-2008$

Table 3: Adjusted Risk Ratios and 95\% Confidence Intervals of Types of Coexisting chronic physical Conditions from Complementary Log-Log Regression on Newly-Diagnosed Depression among Medicaid Beneficiaries with Type 2 Diabetes Mellitus, Multistate Medicaid Claims 20002008 (Reference Group: Concordant only)

Appendix A : ICD-9-CM Codes for Identifying Types of Coexisting chronic physical Conditions.50

Appendix B: Adjusted Risk Ratios and 95\% Confidence Intervals from Complementary Log-Log Regression on Newly-diagnosed Depression among Medicaid Beneficiaries with Type 2 Diabetes Mellitus, Multistate Medicaid Claims 2000-2007

CHAPTER 3: Types of Coexisting Chronic Physical Conditions and Treatment for Depression among Medicaid Beneficiaries with Type 2 Diabetes Mellitus and Newly-diagnosed Depression .......................58

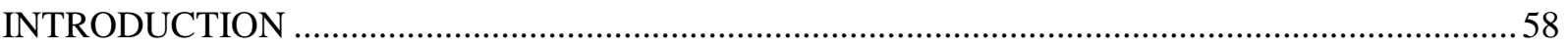

Coexisting Depression among Individuals with Type 2 Diabetes Mellitus (T2DM).........................58

Treatment for Depression among Individuals with T2DM ......................................................... 58

Depression Treatment Patterns among Individuals with T2DM .................................................60

Depression Treatment among Individuals with Coexisting Chronic Physical Conditions ................. 61

Depression Treatment among Individuals with T2DM and Coexisting Chronic Physical conditions 62 Adequate Depression Treatment among Individuals with T2DM and Coexisting Chronic Physical conditions...... 


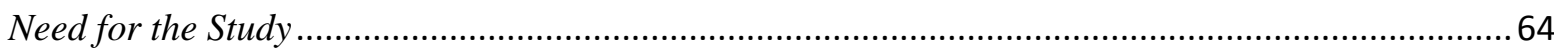

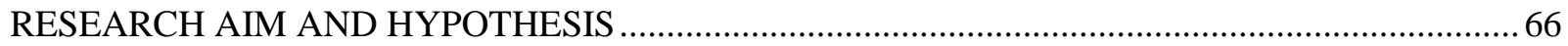

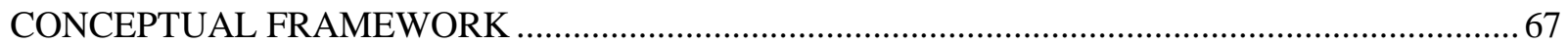

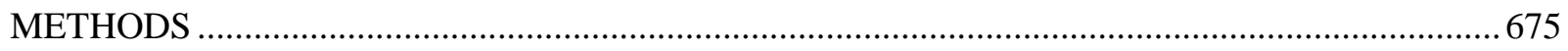

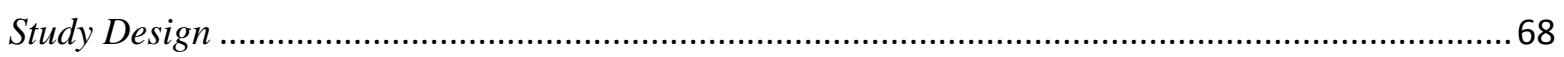

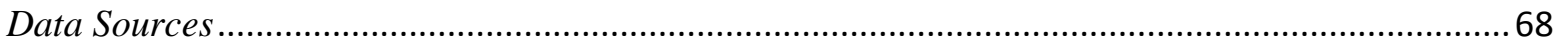

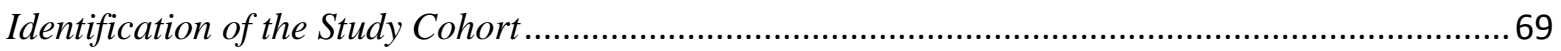

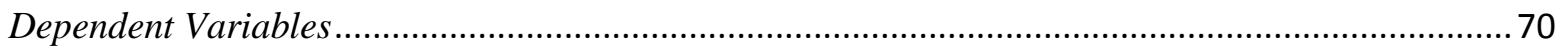

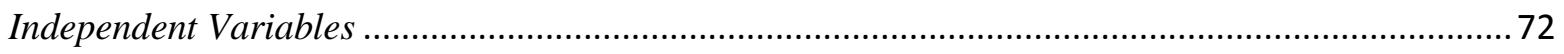

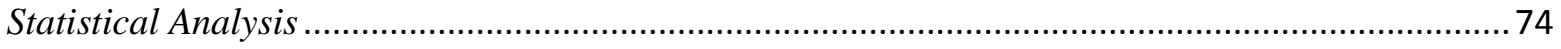

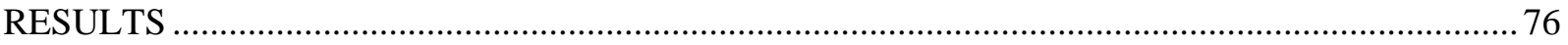

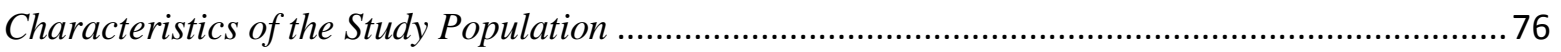

Study Population Characteristics by Types of Coexisting Chronic Physical Conditions .................. 76

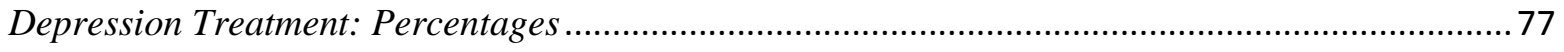

Unadjusted Associations between Types of Coexisting Chronic Physical Conditions and Depression

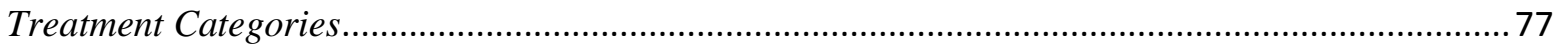

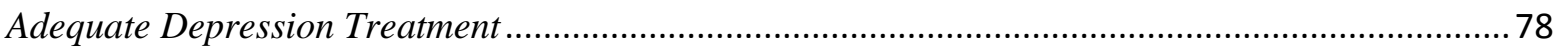

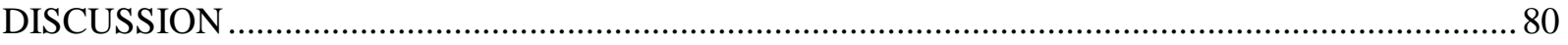

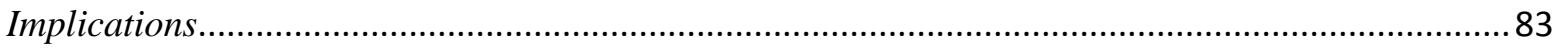

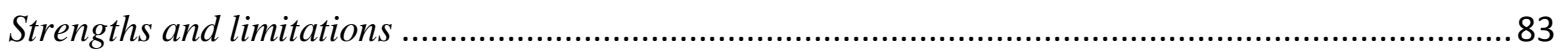

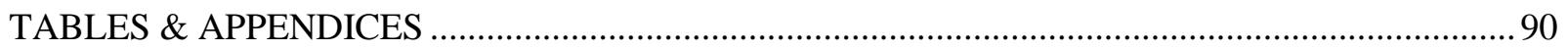

Table 1: Description of the Study Population by Types of Coexisting Chronic Physical Conditions Among Medicaid Beneficiaries with Type 2 Diabetes Mellitus and Newly-diagnosed Depression Multi-state Medicaid Claims Database - 2000 - 2008 ................................................................. 90

Table 2: Percentages of Depression Treatment by Types of Coexisting Chronic Physical Conditions Among Medicaid Beneficiaries with Type 2 Diabetes Mellitus and Newly-diagnosed Depression Multi-state Medicaid Claims Database - 2000 - 2008 ….................................................................... 93

Table 3: Odds Ratios and Adjusted Odds Ratio and 95\% Confidence Intervals of Types of Coexisting Chronic Physical Conditions from Multinomial Logistic Regression on Depression Treatment Categories Among Medicaid Beneficiaries with Type 2 Diabetes Mellitus and Newlydiagnosed Depression Multi-state Medicaid Claims Database - 2000 - 2008.

Appendix A : ICD-9-CM Codes for Identifying Types of Coexisting chronic physical Conditions. 98 Appendix B: Odds Ratios and Adjusted Odds Ratio and 95\% Confidence Intervals of All Independent Variables from Multinomial Logistic Regression on Depression Treatment Categories 
Results presented for Antidepressants Only Among Medicaid Beneficiaries with Type 2 Diabetes Mellitus and Newly-diagnosed Depression Multi-state Medicaid Claims Database - 2000 - 2008100 Appendix C: Odds Ratios and Adjusted Odds Ratio and 95\% Confidence Intervals of All Independent Variables from Multinomial Logistic Regression on Depression Treatment Categories Results presented for Psychotherapy Only Among Medicaid Beneficiaries with Type 2 Diabetes Mellitus and Newly-diagnosed Depression Multi-state Medicaid Claims Database - 2000 - 2008103 Appendix D: Odds Ratios and Adjusted Odds Ratio and 95\% Confidence Intervals of All Independent Variables from Multinomial Logistic Regression on Depression Treatment Categories Results presented for Both Antidepressants and Psychotherapy Among Medicaid Beneficiaries with Type 2 Diabetes Mellitus and Newly-diagnosed Depression Multi-state Medicaid Claims Database $-2000-2008$ 106

CHAPTER 4: Treatment for Depression and Healthcare Expenditures among Medicaid Beneficiaries with Type 2 Diabetes Mellitus and Newly-Diagnosed Depression ............................................................... 109

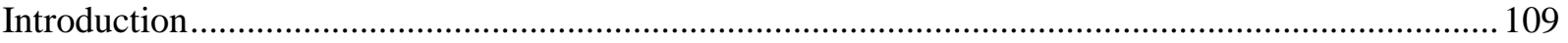

Type 2 Diabetes Mellitus (T2DM), Depression and Healthcare Expenditures ............................... 109

Treatment for Depression and Healthcare Expenditures among Individuals with T2DM ..............109

The Relationship between Depression Treatment and Healthcare Expenditures by Coexisting

Chronic Physical Conditions ................................................................................................... 111

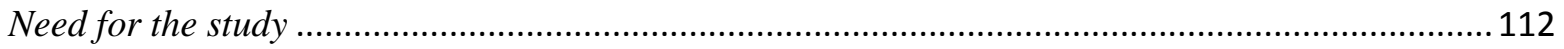

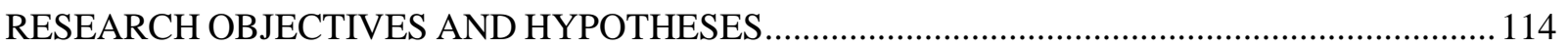

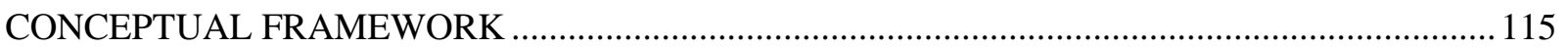

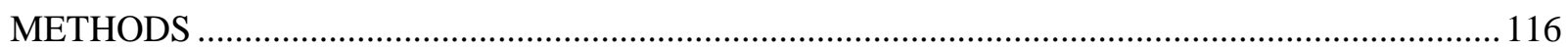

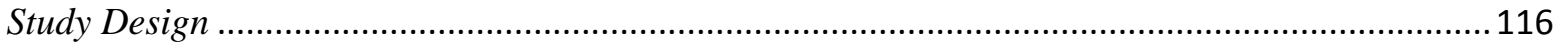

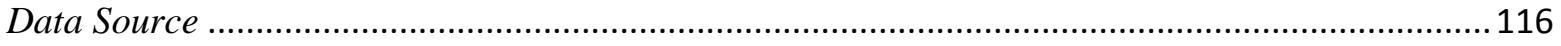

Identification of T2DM and Newly-diagnosed Depression Cohort .............................................117

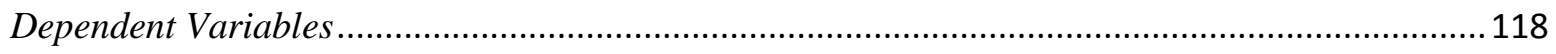

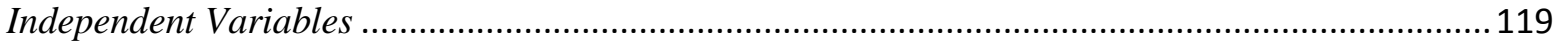

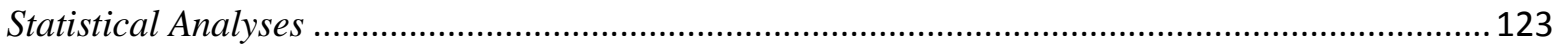

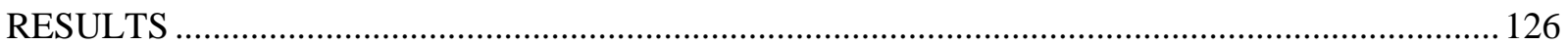

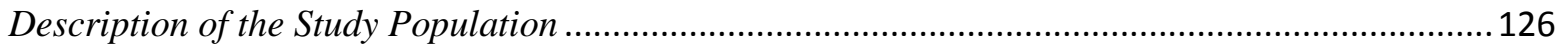

Description of the Study Population by Types of Coexisting Chronic Physical conditions...............126

Unadjusted Association between Types of Coexisting Chronic Physical Conditions and Depression

Treatment Categories.............................................................................................................. 127

Mean Monthly Healthcare Expenditures for Depression Treatment Categories..............................127 
Mean Monthly Healthcare Expenditures for Depression Treatment Categories: by Types of

Coexisting Chronic Physical Conditions

Adjusted Association between Depression Treatment Categories during the Acute Phase and

Healthcare Expenditures.

Adjusted Association between Depression Treatment Categories during the Acute Phase and

Healthcare Expenditures by Types of Coexisting Chronic Physical Conditions ............................ 130

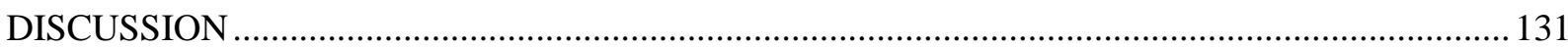

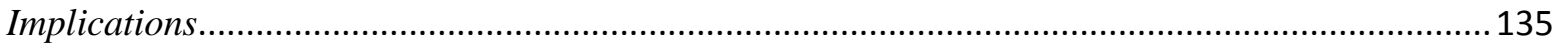

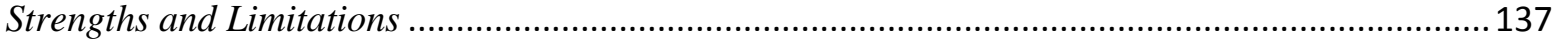

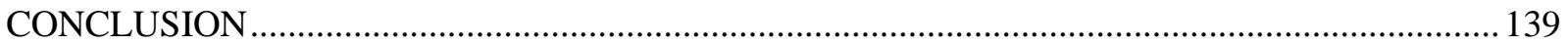

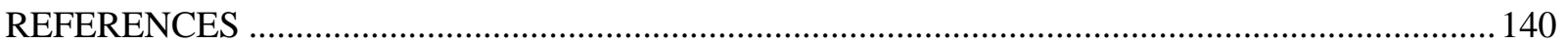

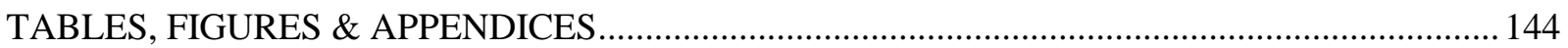

Table 1: Description of the Study Population by Types of Coexisting Chronic Physical Conditions

Among Medicaid Beneficiaries with Type 2 Diabetes Mellitus and Newly-diagnosed Depression

Multi-state Medicaid Claims Database - 2000 - 2008.

Table 2: IPTW Adjusted Association Between Depression Treatment and Healthcare Expenditures, Among Medicaid Beneficiaries with Type 2 Diabetes Mellitus and Newly-diagnosed Depression Multistate Medicaid Claims Database - 2000 - 2008 (Reference Group : No Depression Treatment) ........ 147

Table 3: IPTW Adjusted Association Between Depression Treatment and Healthcare Expenditures Stratified by Types of Coexisting Conditions among Medicaid Beneficiaries with Type 2 Diabetes Mellitus and Newly-diagnosed Depression, Multi-state Medicaid Claims Database - 2000 - 2008, (Reference Group : No Depression Treatment)

Figure 1: Mean IPTW adjusted Total and T2DM-Related Healthcare Expenditures during Follow-up for Medicaid Beneficiaries with T2DM and Newly-Diagnosed Depression

Figure 2: Mean IPTW adjusted Total and T2DM-Related Healthcare Expenditures during Follow-up for Medicaid Beneficiaries with T2DM and Newly-Diagnosed Depression: By Types of Coexisting Chronic Physical Conditions.

Appendix A : ICD-9-CM Codes for Identifying Types of Coexisting chronic physical Conditions... 152

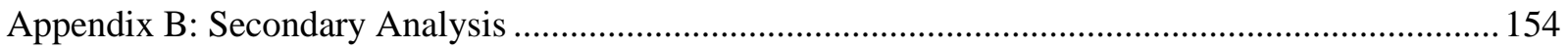

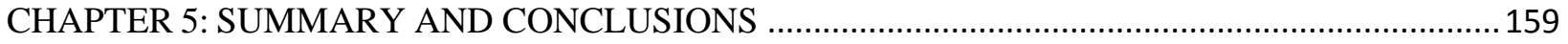

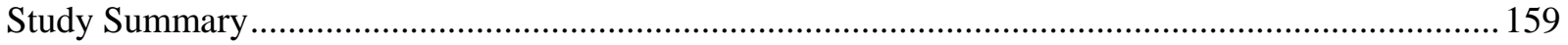

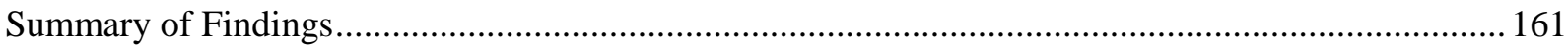

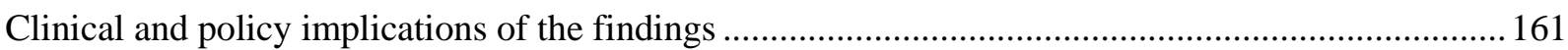

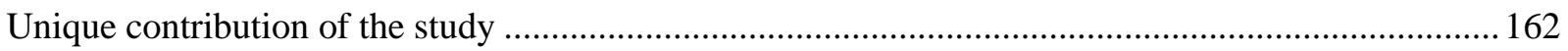

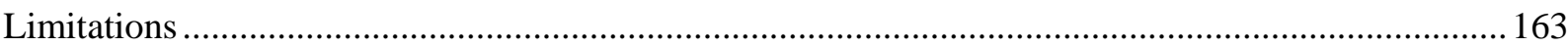

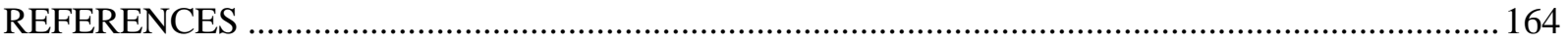


CHAPTER 1 


\section{CHAPTER 1: Background and Significance}

\section{Epidemiology of Type 2 Diabetes and Depression}

Diabetes is a widely prevalent metabolic condition. According to a World Health Organization report recently updated in 2014, 347 million people around the world suffer from diabetes [1]. In the US, based on 2005-2008 National Health and Nutrition Examination Survey, 25.6 million individuals i.e. $11.3 \%$ of the US population who were 20 years or older had diabetes [2]. It has been estimated that among adults with a diagnosis of diabetes, $90 \%-95 \%$ suffer from type 2diabetes mellitus (T2DM) [2, 3].

Depression is a highly disabling, yet very commonly prevalent psychiatric condition with worldwide new diagnosis (i.e. incidence) rates of 3\%. Depression has been reported to be the second leading cause of disability as defined by years lived with disability (YLD) estimates [4]. In the US, over a period of 12 months, $5.28 \%$ of the population has been found to be affected by depression [5]. Coexisting depression leads to significant disease burden in terms of impaired cognition, reduced quality of life, increased mortality risk, social dysfunction and financial burden [6]. Presence of depression among adults with chronic medical illnesses is associated with high risk of mortality, increased disability and poor adherence to self-care regimens.

Adults with T2DM are considered to be a high risk of developing depression. Pooled estimates from two previous meta-analyses [7, 8] examining the association of T2DM and depression have found that the risk of developing depression among individuals with T2DM, range from $15 \%$ (Relative Risk (RR): 1.15; 95\% CI 1.02-1.30) [8] to 24\% (RR: 1.24; 95\% CI 1.09-1.40) [7]. 


\section{Coexisting chronic physical conditions, T2DM and Depression}

Among adults with T2DM, coexisting chronic physical conditions are often the norm rather than exception and presence of such conditions affect the management of T2DM. Adults with T2DM and coexisting chronic physical conditions often experience confusion, frustration and feelings of being overwhelmed, perhaps due to several different and sometimes conflicting recommendations from clinicians with regard to management of their conditions [9]. Therefore, adults with T2DM and coexisting chronic physical conditions are at risk for developing depression. Using the Canadian National Population Health Survey (NPHS), a study found that as compared to the $2.8 \%$ (95\% Confidence Interval (CI):2.2-3.4) rate of new major depressive episodes among those no long term medical condition, those with any long term medical condition had a 4\% (95\% CI: 3.3-4.7) rate of major depressive episode; long term medical conditions included hypertension, food allergies, other allergies, asthma, arthritis/'rheumatism', back problems, migraine headaches, sinusitis, diabetes, heart disease, peptic ulcers/gastritis and chronic bronchitis/emphysema [10]. Another study conducted in the US examined the risk of development of significant depressive symptoms after a new diagnosis of several different chronic conditions [11]. This study found that within 2 years of initial diagnosis, those with cancer were 3.55 times (Hazards Ratio (HR): 3.55; 95\% CI: 2.79-4.52), those with chronic lung disease had 2.21times (HR: 2.21; 95\% CI:1.64-2.97) and those with heart disease had 1.45 times (HR:1.45; 95\% CI: 1.09-1.93) higher risk of developing depressive symptoms [11].

\section{Relationship between types of physical conditions and risk of depression}

It is plausible that adults with T2DM and coexisting chronic physical conditions with similar disease management strategies may be at a lower risk for developing depression compared to those with coexisting chronic physical conditions that may require contradicting 
clinical and self-management strategies. Only six these studies, controlled for types of coexisting chronic physical conditions while estimating the risk of developing depression among adults with T2DM [12-17] as compared to adults with no T2DM. Some of these studies [13] [17] found that coexisting chronic physical conditions were significantly associated with risk of depression, while other studies [12] [14] found that coexisting chronic physical conditions were not significantly associated with risk of depression. De Jonge et al., [16] controlled for the interaction of T2DM and coexisting chronic conditions in their model, but this interaction was not significantly associated with risk of newly diagnosed depression ( $p$-value $=0.26$ ). Maraldi et al., [15] article did not report the estimates on the association between coexisting chronic conditions and newly diagnosed depression, but found that controlling for coexisting conditions, the significant association between T2DM and incident depression disappeared. Therefore, except the one study by De Jonge et al., [16] which controlled for an interaction term, no other study has examined the association between coexisting conditions and newly diagnosed depression among adults with T2DM. However, in this study coexisting conditions were measured by counting the number of medication classes prescribed. Therefore, no existing study has examined whether among individuals with T2DM, types of coexisting chronic physical conditions affect the risk of depression. As a majority of individuals with T2DM live with multiple other coexisting chronic physical conditions, it is essential to examine whether individuals with different types of coexisting chronic physical conditions are at higher risk of developing depression as compared to other groups.

\section{Relationship between types of chronic physical conditions and treatment of depression}

A handful of studies have indirectly examined the association between types of coexisting chronic physical conditions and depression treatment patterns among individuals with 
T2DM. However, coexisting chronic physical conditions in these studies were measured using either comorbidity indices $[18,19]$ or number of coexisting conditions [20]. Two of these studies found that the number of coexisting chronic condition variable or the comorbidity index variable was not significantly associated with receipt of depression treatment $[18,20]$. On the contrary, in the study among Medicaid beneficiaries with T2DM by Sambamoorthi et al.,[19] it was reported that individuals with higher burden from coexisting conditions, were more likely to receive antidepressants compared to those with no burden from coexisting conditions. However, since these studies used comorbidity indices or number of chronic condition variable in their analyses, there is an existing knowledge gap regarding how different types of coexisting conditions may effect depression treatment among individuals with T2DM and coexisting chronic physical conditions.

Additionally, no study has examined the relationship between adequate depression treatment and types of chronic physical conditions, among non-elderly adults with T2DM. Coexisting chronic physical conditions may be associated with whether an individual received adequate depression treatment. For example, many adults with T2DM also have coexisting cardiovascular diseases, physicians may be cautious in prescribing adequate antidepressant treatment for adults with T2DM and coexisting chronic physical conditions such as hypertension and heart disease. As depression treatment often competes with treatment for other conditions, among individuals with multiple coexisting conditions, it is possible that even those who start depression treatment may not get the "adequate" treatment as per guidelines for depression treatment. Adequate depression treatment is one of the quality measures included in National Committee for Quality Assurance's (NCQA) Healthcare Effectiveness Data and Information Set (HEDIS). These quality measures are used by various health plans including Medicaid and other 
organizations in the US for the purpose of accreditation, accountability and quality improvement. Therefore, there is a need to analyze the association of adequate depression treatment with type coexisting chronic physical conditions, among individuals with T2DM and a newly diagnosed depression.

Economic benefits of treating depression and its association with types of chronic physical conditions

Studying the economic benefits of treating depression among adults with T2DM has become a necessity because of rising healthcare expenditures of T2DM and policy efforts towards cost-containment strategies. According to the American Diabetes Association, the direct medical care expenditures of diagnosed diabetes has increased from \$245 billion in 2012 from \$174 billion in 1997[21]. Among adults with diabetes, healthcare expenditures in specific populations are higher than others. For example, among individuals with diabetes, those with coexisting depression could have mean total annual healthcare expenditures 4.5 times higher $(\$ 247,000,000)$ compared to those without depression $(\$ 55,000,000)$ [22]. Healthcare payers need to understand if depression management is associated with reduced healthcare expenditures in presence of T2DM and multiple coexisting conditions. Randomized controlled trials [23-25] have shown that among individuals with T2DM, depression treatment with pharmacotherapy and/or psychotherapy using stepped care or algorithm based approach in collaborative care settings are cost-effective. However, the economic benefits of treating depression among adults with T2DM in real-world practices and how the relationship varies by types of coexisting chronic physical conditions are not known.

\section{Need for Using Non-Elderly Medicaid Population}

It is particularly important to study this association in non-elderly population given that, in recent years, the prevalence of multiple coexisting chronic conditions, which has traditionally 
been thought of as a problem in the elderly population, has been increasing among non-elderly adults. In the US, among adults in the age group 45-64 years, the prevalence of multiple coexisting chronic conditions has grown from $24.5 \%$ in 2004 to $28.1 \%$ in in 2010 [26]. In the US, State Medicaid Plans are a major provider of health insurance among non-elderly adults. As of 2010, 27\% of US adults, 20-64 years of age were covered by Medicaid [16]. Adults with diabetes are disproportionately covered by Medicaid and four out of five Medicaid beneficiaries with diabetes suffer from a coexisting chronic physical condition. It has been reported that among Medicaid enrollees, those with diabetes spend 2.5 times more than those without diabetes [27]; 20\% of Medicaid enrollees are diagnosed with depression [28, 29]. According to Agency of Healthcare Research and Quality 2011 statistical brief, mood disorders which includes depression ranks top most among the ten conditions for 30 day readmission in Medicaid resulting in 41,600 readmissions and costing \$286 million [30]. Depression is also a major driver for poor health outcomes and future healthcare expenditures in T2DM [31]. Given such compelling statistics, it is apparent that Medicaid data provides a rich opportunity to conduct research of both depression and T2DM, and can lead to important findings that might influence clinical practice and policy. Therefore, delineating relationship between depression, its treatment and healthcare expenditures among Medicaid enrollees with T2DM and understanding how types of coexisting chronic physical conditions impact this association is necessary in the high risk Medicaid population.

\section{Theoretical Framework Used to Categorize Types Of Coexisting Chronic Physical Conditions}

Existing theoretical framework developed by Piette and Kerr has categorized forty-four different chronic physical illnesses commonly coexisting with T2DM into concordant, discordant and dominant conditions using ICD-9-CM codes [44]. Piette and Kerr had developed this novel 
theoretical framework for categorizing coexisting chronic physical conditions based on how these conditions had an impact on the medical care, self-management, and healthcare outcomes of an individual with T2DM. This framework suggested that studies which used one-dimensional scores or number of coexisting conditions to assess the impact of coexisting conditions on T2DM care, implicitly assumed that all conditions have similar impact on T2DM care. However, such studies fail to identify whether characteristics specific to coexisting conditions impact patient priorities for T2DM care. Based on typologies of coexisting conditions, the theoretical framework developed by Piette and Kerr identified coexisting conditions among individuals with T2DM as: dominant (conditions whose severity eclipses all other conditions' management plans such as metastatic cancer), concordant (conditions that overlap with T2DM in their pathophysiology and management plans such as cardiovascular diseases) and discordant (conditions with unrelated pathophysiology or management plans such as musculoskeletal disorders) [32].

Though this study does not assess outcomes related to T2DM, the same theory may be applied to development of newly-diagnosed depression. Based on disease pathophysiology pathophysiology and similarity or differences with T2DM management, dominant, concordant and discordant conditions may have different risks associated with development of newlydiagnosed depression, its treatment and economic outcomes of depression treatment. Therefore, based on Piette and Kerr's framework, a hierarchical classification [33] was used to categorize forty four physical conditions which are found commonly coexisting with. Dominant conditions were given priority in the hierarchical classification because such conditions eclipse the management of other health conditions. Among those without dominant conditions, concordant and discordant conditions were identified. The types of coexisting chronic physical conditions 
were classified as: 1) Dominant Conditions, 2) Concordant only, 3) Discordant only and 4) Both concordant and discordant. The conditions were identified using ICD-9-CM codes and are presented in Appendix A.

\section{Research Objectives and Hypotheses}

Aim 1: Among non-elderly (18-64 years) Medicaid beneficiaries with T2DM, examine whether risk of newly-diagnosed depression vary by types of coexisting chronic physical conditions. Hypothesis 1.1: Adults with dominant, both concordant and discordant conditions and discordant conditions only would have higher risk of newly-diagnosed depression as compared to individuals with concordant conditions only

Aim 2: Objective 1.1: Examine the association between types of coexisting chronic physical conditions and depression treatment (antidepressants only, psychotherapy only, both antidepressants and psychotherapy and none), after adjusting for other covariates among nonelderly (18-64 years) fee-for-service Medicaid beneficiaries with T2DM and newly-diagnosed depression,

Aim2: Hypothesis 1.1: As compared to individuals with discordant conditions only, those with concordant conditions would be less likely to receive treatment with antidepressants.

Aim 2: Objective 1.2: Evaluate the association between types of coexisting chronic physical conditions and receipt of adequate depression treatment (yes, no), after adjusting for other covariates among non-elderly (18-64 years) fee-for-service Medicaid beneficiaries with T2DM and newly-diagnosed depression who received either antidepressants or psychotherapy. Aim 2: Hypothesis 1.2: As compared to individuals with discordant conditions only, those with dominant conditions would be less likely to receive adequate depression treatment. 
Aim 3: Objective 3.1: Among non-elderly Medicaid beneficiaries with T2DM and newlydiagnosed depression, examine the association depression treatment categories and total and T2DM-related healthcare care expenditures.

Hypothesis 3.1: As compared to those with no depression treatment, those with depression treatment with only antidepressants, only psychotherapy or both, will have negative associations with total and T2DM-related healthcare expenditures as compared no depression treatment. Aim 3.2: Among non-elderly Medicaid beneficiaries with T2DM and newly-diagnosed depression, examine whether the association between depression treatment and total and T2DMrelated medical care expenditures vary by types of coexisting chronic physical conditions. Hypothesis 3.2.1: As compared to no depression treatment, treatment within both antidepressants and psychotherapy will reduce total and T2DM related healthcare expenditures across all conditions.

Hypothesis 3.2.2: Depression treatment with only antidepressants will not be associated with T2DM related healthcare expenditures among those with concordant conditions only. 


\section{REFERENCES}

1. World Health Organization. Fact Sheet: Diabetes. Available at: http://www.who.int/mediacentre/factsheets/fs312/en/.

2. Preskorn, S.H., Antidepressant drug selection: criteria and options. J Clin Psychiatry, 1994. 55 Suppl A: p. 6-22; discussion 23-4, 98-100.

3. Shaw, J.E., R.A. Sicree, and P.Z. Zimmet, Global estimates of the prevalence of diabetes for 2010 and 2030. Diabetes Res Clin Pract, 2010. 87(1): p. 4-14.

4. $\quad$ Ferrari, A.J., et al., Burden of depressive disorders by country, sex, age, and year: findings from the global burden of disease study 2010. PLoS Med, 2013. 10(11): p. e1001547.

5. Hasin, D.S., et al., Epidemiology of major depressive disorder: results from the National Epidemiologic Survey on Alcoholism and Related Conditions. Arch Gen Psychiatry, 2005. 62(10): p. 1097-106.

6. Lepine, J.P. and M. Briley, The increasing burden of depression. Neuropsychiatr Dis Treat, 2011. 7(Suppl 1): p. 3-7.

7. Nouwen, A., et al., Type 2 diabetes mellitus as a risk factor for the onset of depression: a systematic review and meta-analysis. Diabetologia, 2010. 53(12): p. 2480-6.

8. Mezuk, B., et al., Depression and type 2 diabetes over the lifespan: a meta-analysis. Diabetes Care, 2008. 31(12): p. 2383-90.

9. Beverly, E.A., et al., Perceived challenges and priorities in co-morbidity management of older patients with Type 2 diabetes. Diabet Med, 2011. 28(7): p. 781-4.

10. Patten, S.B., Long-term medical conditions and major depression in a Canadian population study at waves 1 and 2. J Affect Disord, 2001. 63(1-3): p. 35-41.

11. Polsky, D., et al., Long-term risk for depressive symptoms after a medical diagnosis. Arch Intern Med, 2005. 165(11): p. 1260-6.

12. Aarts, S., et al., Diabetes mellitus type II as a risk factor for depression: a lower than expected risk in a general practice setting. Eur J Epidemiol, 2009. 24(10): p. 641-8.

13. Brown, L.C., et al., Type 2 diabetes does not increase risk of depression. CMAJ, 2006. 175(1): p. 42-6.

14. Golden, S.H., et al., Examining a bidirectional association between depressive symptoms and diabetes. JAMA, 2008. 299(23): p. 2751-9.

15. Maraldi, C., et al., Diabetes mellitus, glycemic control, and incident depressive symptoms among 70- to 79-year-old persons: the health, aging, and body composition study. Arch Intern Med, 2007. 167(11): p. 1137-44.

16. de Jonge, P., et al., Prevalent and incident depression in community-dwelling elderly persons with diabetes mellitus: results from the ZARADEMP project. Diabetologia, 2006. 49(11): p. 2627-33.

17. O'Connor, P.J., et al., Does diabetes double the risk of depression? Ann Fam Med, 2009. 7(4): p. 328-35.

18. Tiwari, A., et al., Guideline-consistent antidepressant treatment patterns among veterans with diabetes and major depressive disorder. Psychiatr Serv, 2008. 59(10): p. 1139-47.

19. Sambamoorthi, U., et al., Diabetes and depression care among medicaid beneficiaries. J Health Care Poor Underserved, 2006. 17(1): p. 141-61. 
20. Sambamoorthi, U., et al., Depression treatment patterns among women veterans with cardiovascular conditions or diabetes. World Psychiatry, 2010. 9(3): p. 177-82.

21. American Diabetes, A., Economic costs of diabetes in the U.S. in 2012. Diabetes Care, 2013. 36(4): p. 1033-46.

22. Egede, L.E., D. Zheng, and K. Simpson, Comorbid depression is associated with increased health care use and expenditures in individuals with diabetes. Diabetes Care, 2002. 25(3): p. 464-70.

23. Katon, W.J., et al., Long-term effects on medical costs of improving depression outcomes in patients with depression and diabetes. Diabetes Care, 2008. 31(6): p. 1155-9.

24. Katon, W., et al., Cost-effectiveness and net benefit of enhanced treatment of depression for older adults with diabetes and depression. Diabetes Care, 2006. 29(2): p. 265-70.

25. Simon, G.E., et al., Cost-effectiveness of systematic depression treatment among people with diabetes mellitus. Arch Gen Psychiatry, 2007. 64(1): p. 65-72.

26. Ward, B.W. and J.S. Schiller, Prevalence of multiple chronic conditions among US adults: estimates from the National Health Interview Survey, 2010. Prev Chronic Dis, 2013. 10: p. E65.

27. The Kaiser Family Foundation. 2012.The Role of Medicaid for People with Diabetes. Avaiable at: http://kaiserfamilyfoundation.files.wordpress.com/2013/01/8383_d.pdf. Accessed on December 29, 2014

28. Adelmann, P.K., Mental and substance use disorders among Medicaid recipients: prevalence estimates from two national surveys. Adm Policy Ment Health, 2003. 31(2): p. 111-29.

29. Pratt, L.A. and D.J. Brody, Depression in the United States household population, 2005 2006. NCHS Data Brief, 2008(7): p. 1-8.

30. Agency for Healthcare Research and Quality. Statistical Brief \#172. 2011. Conditions With the Largest Number of Adult Hospital Readmissions by Payer, 2011. Avaiable at: http://www.hcup-us.ahrq.gov/reports/statbriefs/sb172-Conditions-ReadmissionsPayer.pdf. Accessed on December 29, 2014.

31. $\{$ Gilmer, G., T. P., et al., Predictors of health care costs in adults with diabetes. Diabetes Care, 2005. 28(1): p. 59-64.

32. Piette, J.D. and E.A. Kerr, The impact of comorbid chronic conditions on diabetes care. Diabetes Care, 2006. 29(3): p. 725-31.

33. Pentakota, S.R., et al., Does diabetes care differ by type of chronic comorbidity?: An evaluation of the Piette and Kerr framework. Diabetes Care, 2012. 35(6): p. 1285-92. 
CHAPTER 2 


\section{CHAPTER 2: Type of Coexisting Chronic Physical Conditions and the Risk of Newly- diagnosed Depression among Medicaid Beneficiaries with Type 2 Diabetes}

\section{Introduction}

\section{Epidemiology of Diabetes}

Diabetes is a widely prevalent metabolic condition. According to a World Health Organization report recently updated in 2014, 347 million people around the world suffer from diabetes [1]. In the US, based on 2005-2008 National Health and Nutrition Examination Survey, 25.6 million individuals i.e. $11.3 \%$ of the US population who were 20 years or older had diabetes [2]. It has been estimated that among adults with a diagnosis of diabetes, $90 \%-95 \%$ suffer from type 2diabetes mellitus (T2DM) [2,3].

\section{Epidemiology of Depression}

Depression is a highly disabling, yet very commonly prevalent psychiatric condition with worldwide new diagnosis (i.e. incidence) rates of 3\%. Depression has been reported to be the second leading cause of disability as defined by years lived with disability (YLD) estimates [4]. In the US, over a period of 12 months, $5.28 \%$ of the population has been found to be affected by depression [5]. With the elimination of "bereavement exclusion" for clinical diagnosis of depression under Diagnostic and Statistical Manual-5 (DSM-5), the rates of new diagnosis of depression is expected to rise further [6].

\section{T2DM and Risk of Newly-diagnosed Depression}

Adults with T2DM are at a high risk of developing depression. Prospective and retrospective studies have examined the risk of developing depression among adults with T2DM by comparing them to those without T2DM [7-20]. These studies were based on data from different countries; four were from the Netherlands [7, 8, 10, 20], one each from Norway [9], Korea [11], Canada [12] and Taiwan[13] and six from the US [14-19] . Rates of newly- 
diagnosed depression in these studies varied because of differences in study design, lengths of follow-up, settings and measurement of depression. The minimum length of follow-up was 2 years [11] and the maximum was 12 years [12]. Twelve [7-11, 14-18, 20] of these studies had a prospective design and the remaining three were retrospective observational in design $[12,13$, 19]; two studies assessed rates of newly-diagnosed depression after combining data from two different time points (years 2 and 5) to [7, 8]. Some studies identified depression using diagnosis scales such as the Center for Epidemiologic Studies Depression Scale (CES-D) [7, 16, 17], Edinburgh Depression Scale (EDS) [20], Beck Depression Inventory (BDI)[14], Mental Health Index (MHI-5), a five-item subscale of the Short-Form 36 Health Status [18], Hospital Anxiety and Depression Scale - Depression (HADS-D) [9], Geriatric Mental State B3 Diagnostic Schedule with application of the Automated Geriatric Examination for Computer-Assisted Taxonomy algorithm (GMS-AGECAT) [8]; others used inpatient or outpatient diagnosis codes for depression as identified by physicians [13], alone or in conjunction with antidepressant drug use $[12,19]$, to identify newly-diagnosed depression.

The adjusted odds ratio (AOR) and 95\% confidence interval (CI) estimates for newlydiagnosed depression in studies using diagnosis scales varied from (AOR: 0.73; 95\% CI: $0.41-$ 1.30) [14] to (AOR: 1.54; 95\% CI: 1.13-2.09) [17] and the estimates ranged from (AOR: 1.10; 95\% CI: 1.01-1.19) [12] to (AOR:1.48; 95\% CI: 1.35-1.63) [19] for studies using diagnosis codes. Meta-analyses that have included majority of these studies have concluded that the risk of developing depression among individuals with T2DM, ranged from 15\% (Adjusted Relative Risk (ARR): 1.15 ; 95\% Confidence Interval: $1.02-1.30$ ) [21] to 24\% (ARR: $1.24 ; 95 \%$ CI 1.09-1.40) [22]. The Mezuk et al., meta-analysis [23] included 7 studies and the other meta-analysis by Nouwen et al., which was published later, included 11 studies [22]. 
Many of the above-mentioned studies did not differentiate between depressive symptoms and major depression. Often self-reported depression based on diagnostic scales was used [7, 9, 14-17]; newly-diagnosed depression was identified as appearance of "depressive symptoms", defined by a pre-validated cut-off score on the diagnosis scales, e.g. $>=16$ on CES-D scale, $>=12$ on EDS, $>=8$ on HADS-D, $<=52$ on MHI-5. Self-reports may overestimate the extent of depressive symptoms and there is discordance between rates of newly-diagnosed depression identified by scores on self-reported depression scales and depression identified by diagnostic schedules such as Diagnostic Statistical Manual (DSM-IV) [24]. On the other hand, the study by Brown et al., [12] identified depression by including use of antidepressants along with physician recognized clinical diagnosis code for depression. However, studies have reported that two thirds of individuals with depression is often not treated [25]. Therefore, use of antidepressants as a criterion for identifying depression may lead to underestimated rates. Additionally, these studies compared risk of depression among those with T2DM as compared to those without T2DM. None of them examined whether risk of depression differed among subgroups of individuals with T2DM and coexisting conditions.

\section{Coexisting Chronic Physical Conditions among Adults withT2DM}

Among adults with T2DM coexisting chronic physical conditions are often the norm rather than the exception and presence of such conditions affect the management of T2DM. According to Medical Expenditure Panel Survey data, majority of adults (88.6\%) with T2DM in the United States (US) have at least one chronic condition and 15\% have reported having four or more chronic conditions [26]. Based on similarity or difference from T2DM management and pathophysiology, types of coexisting chronic physical conditions among individuals with T2DM may be concordant (conditions that overlap with T2DM in their pathophysiology and 
management plans such as cardiovascular diseases), discordant (conditions with unrelated pathophysiology or management plans such as musculoskeletal disorders), or dominant (conditions whose severity eclipses all other conditions' management plans such as metastatic cancer) [27]. Among 42,826 Veterans with T2DM, it has been found that $12 \%$ had dominant conditions, $13 \%$ had concordant only, $30 \%$ had discordant only and $25 \%$ had both concordant and discordant [28].There is evidence that types of coexisting chronic physical conditions affect T2DM management [28]

\section{Coexisting Chronic Physical Conditions and Risk of Depression}

Coexisting depression in adults with chronic physical conditions often lead to impaired cognition, reduced quality of life, increased mortality, social dysfunction and financial burden [29]. Therefore, individuals with coexisting chronic physical conditions are at particularly high risk of developing depression. Using the Canadian National Population Health Survey, a study found that rates of new major depressive episodes among those with no long term medical conditions were lower as compared to those with any long term medical condition $(2.8 \%$ (95\% CI:2.2-3.4) vs 4\% (95\% CI: 3.3-4.7); long term medical conditions included hypertension, food allergies, other allergies, asthma, arthritis/'rheumatism’, back problems, migraine headaches, sinusitis, diabetes, heart disease, peptic ulcers/gastritis and chronic bronchitis/emphysema [30]. Another study conducted in the US examined the risk of development of significant depressive symptoms after a new diagnosis of several different chronic conditions [15]. This study found that within 2 years of initial diagnosis, the risk of developing depressive symptoms was, 3.55 times higher among with cancer (Hazards Ratio (HR): 3.55; 95\% CI: 2.79-4.52), 2.21 times higher among those with chronic lung disease had (HR: 2.21; 95\% CI:1.64-2.97) and 1.45 times higher among those with heart disease (HR:1.45; 95\% CI: 1.09-1.93) [15]. 


\section{Coexisting Chronic Physical Conditions among Adults with T2DM and Risk of Depression}

Adults with T2DM and coexisting chronic physical conditions often experience confusion, frustration and feelings of being overwhelmed, perhaps due to several different and sometimes conflicting recommendations from clinicians with regard to management of their

conditions [31]. Such feelings may eventually lead to poor emotional health and development of depression. To the best of our knowledge no study has evaluated the risk of newly-diagnosed depression by types of coexisting chronic physical conditions among adults with T2DM. Therefore, available evidence from studies that have examined the risk of newly-diagnosed depression after controlling for coexisting conditions, among adults with T2DM as compared to those without T2DM, has been parsed.

Only six of the fifteen studies that examined the risk of depression among adults with T2DM controlled for types of co-existing chronic physical conditions in their multivariable analyses $[8,10,12,16,17,19]$. Some of these studies [12] [19] found that coexisting chronic physical conditions were significantly associated with an increased risk of depression, while other studies [10] [17] found that coexisting chronic physical conditions were not significantly associated with risk of depression. Maraldi et al., [16] did not report the estimates of the association between coexisting conditions and newly-diagnosed depression, but found that controlling for coexisting conditions, the significant association between T2DM and newlydiagnosed depression disappeared. The study by de Jonge et al., [8] controlled for the interaction of T2DM and coexisting conditions, but this interaction was not significantly associated with risk of newly-diagnosed depression ( $\mathrm{p}$-value $=0.26$ ). This may indicate that the risk of newlydiagnosed depression does not vary by coexisting conditions. However, in this study coexisting conditions were measured by counting the number of medication classes prescribed. Therefore, 
except the one study by de Jonge et al., [8] which controlled for an interaction term, no other study has examined the association between coexisting conditions and newly-diagnosed depression among adults with T2DM.

\section{Need for the Study}

Presence of depression among adults with chronic medical illnesses is associated with high risk of mortality, increased disability, poor adherence to self-care regimens and high health care expenditures [32]. Identification of groups of individuals at high risk of depression can help in prevention, timely diagnosis and management of the disease. From the literature discussed, it is apparent that individuals with T2DM are at increased risk of developing depression. Presence of coexisting conditions may also increase the risk of depression. Since, majority of individuals with T2DM live with multiple other coexisting chronic physical conditions [26], it is essential to examine whether subgroups of individuals with T2DM and particular types of coexisting chronic physical conditions are at higher risk of developing depression as compared to other groups. However, with the conspicuous absence of studies relating to association between types of coexisting chronic physical conditions in adults with T2DM and risk of depression, a significant knowledge gap exists in this area. Therefore, this study intends to fill this knowledge gap by examining the risk of depression for different types of coexisting chronic physical conditions among adults with T2DM.

This study focused on adults with T2DM whose healthcare expenses are covered by state Medicaid programs. There are several reasons to examine this association among Medicaid enrollees. In the US, State Medicaid Plans are a major provider of health insurance among nonelderly adults. As of 2010, 27\% of US adults, 20-64 years of age were covered by Medicaid outlaying $68 \%$ of federal spending [8]. Adults with diabetes are disproportionately covered by 
Medicaid and four out of five Medicaid beneficiaries with diabetes suffer from a coexisting chronic physical condition. Medicaid enrollees with diabetes spend more than 2.5 times compared to those without diabetes [33] and 20\% of Medicaid enrollees are diagnosed with depression [34, 35]. Mood disorders including depression ranks top most among the ten conditions for 30 day readmission in Medicaid programs and costs $\$ 286$ million [36]. Additionally, depression contributes heavily towards poor health outcomes and future healthcare costs in T2DM [37]. Based on these statistics, it can be seen that Medicaid data provides a rich opportunity to conduct research of both depression and T2DM, and can lead to important findings that might influence clinical practice and policy.

This study identified newly-diagnosed depression from International Classification of Diseases $9^{\text {th }}$ Revision ie.ICD-9-CM codes and mainly focused on new diagnoses of major depression. The focus on major depression was because, specific treatment guidelines are available for major depression, while treatment guidelines for minor depression are not clear [38]. Additionally, depression diagnosis from administrative claims data is cheaper compared to resource intensive prospective data collection. Additionally, these diagnosis codes have been identified by Healthcare Effectiveness Data and Information Set, a tool used by $90 \%$ of US healthcare plans including Medicaid [23]. 


\section{RESEARCH AIM AND HYPOTHESIS}

Aim 1: Among non-elderly (18-64 years) Medicaid beneficiaries with T2DM, examine whether risk of newly-diagnosed depression vary by types of coexisting chronic physical conditions.

Hypothesis 1.1: Adults with dominant, both concordant and discordant conditions and discordant conditions only would have higher risk of newly-diagnosed depression as compared to individuals with concordant conditions only 


\section{CONCEPTUAL FRAMEWORK}

An adapted version of the conceptual framework on Determinants of Health Model was used to study the risk factors contributing to chronic diseases. In this framework, a variety of factors may influence the health and well-being of individuals including development of diseases. These factors include environmental factors, geographical location, socioeconomic characteristics, knowledge, attitudes and beliefs, health behaviors, psychological factors, safety factors, biomedical factors and individual physical and psychological makeup. This study focused on biomedical factors i.e. types of coexisting chronic physical condition as risk factor disease i.e. newly-diagnosed depression, after controlling for other factors which were chosen based on the this framework and the availability of variables in the dataset. The following figure presents the adapted theoretical framework used in this study.

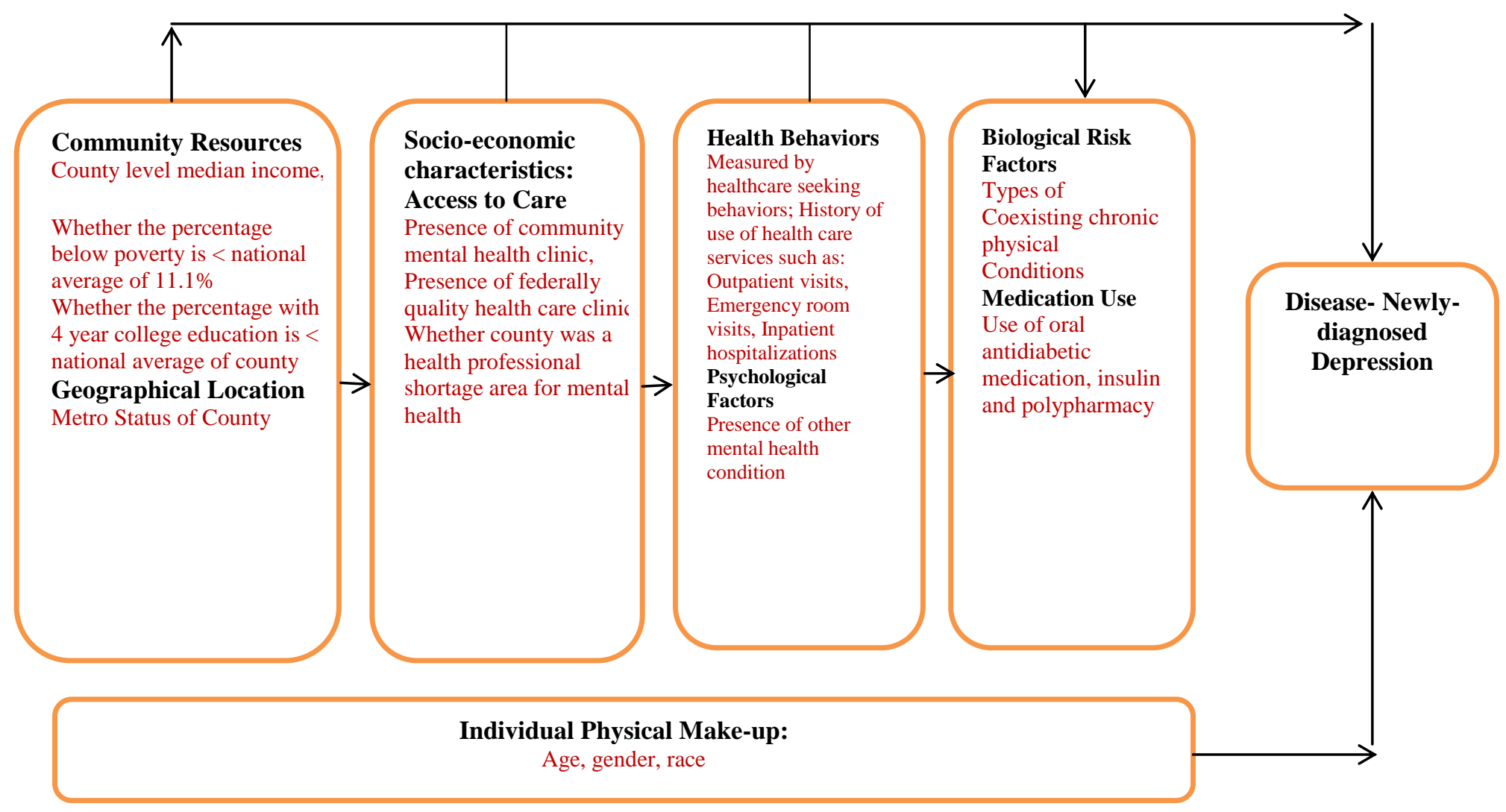




\section{METHODS}

\section{Study Design}

A retrospective cohort study design was used for this study. A cohort of Medicaid beneficiaries with T2DM who were free of depression during baseline were identified. This cohort was then followed in to the following calendar year. Those who received a diagnosis of depression in the following year were considered as having newly-diagnosed depression. Therefore, individuals classified as having a newly-diagnosed depression had at least a 365 day period free of any history of depression diagnosis or antidepressant use.

Among those with newly-diagnosed depression, the date of diagnosis was the "index date". Among individuals with no newly-diagnosed depression, the index date was randomly chosen from the dates of inpatient and outpatient services received by an individual during the calendar year. Baseline period was defined as 12 months prior to the index date. Coexisting chronic physical conditions and health care utilization variables were identified during this baseline period.

\section{Data Sources}

\section{Medicaid Analytic Extract (MAX) Files:}

The Centers for Medicare and Medicaid Services prepares the MAX files. These files comprise of enrollment ("personal summary"), inpatient claims, other therapy medical claims, and pharmacy claims files of Medicaid beneficiaries. These files were linked by beneficiaries' unique identification number. Information on Medicaid eligibility and demographics characteristics were extracted from the personal summary files. Information on diagnoses, use of services and Medicaid payments were identified from the other claims files. This study used 8 years Medicaid data (2000-2007) from three states: New York (NY), Texas (TX), Illinois (IL). 
Medicaid data use agreement policy specifies that cell sizes less than eleven cannot be displayed and percentages or other mathematical formulas may not be used if the result in display has cell sizes less than eleven [39]. To ensure that dependent and all independent variable categories had enough sample sizes, multiple years of Medicaid data were combined.

Area Health Resource File (AHRF):

The AHRF is a publicly available data file provided by Department of Health and Human Services [40] which contains county level information on more than six thousand variables. The Medicaid files were linked with ARF files with five digit county identification variable. This study used AHRF variables such as median household income, percent below poverty level and percent with college education in a county, whether the county of residence of the enrollee had been designated as a health professional shortage area (HPSA) for mental health services, metro status of the county, presence of a Community Mental Health Clinic (CMHC) and a Federally Qualified Healthcare Clinic (FQHC) in the county.

Identification of Study Cohort: Individuals with T2DM and Free of Depression at Baseline

Using Medicaid data from 2000-2007, seven 2-year cohorts (2000-01, 2001-02, 2002-03, 2003-04, 2004-05, 2005-06, 2006-07) were identified. Those with at least one inpatient visit or two or more physician outpatient visits (which were at least 30 days apart) with a primary or secondary diagnosis of International Classification of Diagnosis Codes 9th Revision (ICD-9CM) codes: $250 . x 0$ or $250 . x 2$, were identified as having T2DM. The study population excluded individuals with a diagnosis for depression and antidepressant medication use, in the calendar year. The eligible study population was then followed into the subsequent calendar year to identify newly-diagnosed depression. 
Exclusion criteria for the study cohort were: (1) not having a diagnosis of at least one of the forty-four coexisting chronic physical conditions as identified by ICD-9-CM codes included in the appendix, during the baseline (2) no use of any inpatient or outpatient services during the subsequent calendar year i.e. the calendar year in which newly-diagnosed depression was identified (2) no continuous Medicaid eligibility and (3) enrollment in Medicare managed care at any point during the observation period; and (4) died during the study period.

\section{Dependent Variable}

\section{Newly-Diagnosed Depression:}

Among individuals with T2DM, no diagnosis of depression and no antidepressant use in a calendar year, those with at least one inpatient or outpatient diagnosis [41] corresponding to ICD9 CM codes: 296.2 (major depressive disorder, single episode), 296.3 (major depressive disorder, recurrent episode), 311 (depression not elsewhere classified), 309.1 (prolonged depressive reaction), 300.4 (neurotic depression) and 298.0 (depressive type psychosis) in the subsequent calendar year, were identified as having newly-diagnosed depression [42, 43]. These ICD-9-CM codes were recommended by National Committee for Quality Assurance and had been used in previous studies to identify depression among Medicaid enrollees [42-44]. These diagnosis codes are also widely used by health plans to identify depression [38].

\section{Independent Variables}

\section{Key Independent Variable: Types of coexisting chronic physical Conditions:}

Piette and Kerr had developed a novel theoretical framework for categorizing coexisting chronic physical conditions based on how these conditions had an impact on the medical care, self-management, and healthcare outcomes of an individual with T2DM. This framework suggested that studies which used one-dimensional scores or number of coexisting conditions to 
assess the impact of coexisting conditions on T2DM care, implicitly assumed that all conditions have similar impact on T2DM care. However, such studies fail to identify whether characteristics specific to coexisting conditions impact patient priorities for T2DM care. Based on typologies of coexisting conditions, the theoretical framework developed by Piette and Kerr identified coexisting conditions among individuals with T2DM as: dominant (conditions whose severity eclipses all other conditions' management plans such as metastatic cancer), concordant (conditions that overlap with T2DM in their pathophysiology and management plans such as cardiovascular diseases) and discordant (conditions with unrelated pathophysiology or management plans such as musculoskeletal disorders) [27].

Though this study does not assess outcomes related to T2DM, the same theory may be applied to development of newly-diagnosed depression. Based on disease pathophysiology pathophysiology and similarity or differences with T2DM management, dominant, concordant and discordant conditions may have different risks associated with development of newlydiagnosed depression. Therefore, based on Piette and Kerr's framework, a hierarchical classification [28] was used to categorize forty four physical conditions which are found commonly coexisting with T2DM and this variable was to examine the association between types of coexisting chronic physical conditions and risk of newly-diagnosed depression. These conditions were identified using ICD-9-CM codes and are presented in Appendix A. Dominant conditions were given priority in the hierarchical classification because such conditions eclipse the management of other health conditions. Among those without dominant conditions, concordant and discordant conditions were identified. The types of coexisting chronic physical conditions were classified as: 1) Dominant Conditions, 2) Concordant only, 3) Discordant only and 4) Both concordant and discordant. 


\section{Other independent variables:}

The other independent variables were selected based on the Determinants of Health Model. Individual Physical Make-up: The variables included were: age, sex, race/ethnicity [Whites, African Americans, Hispanics or other races]);

Psychological Factors: Defined by presence of other mental health conditions in the baseline. The other mental health conditions included bipolar disorder, schizophrenia, post-traumatic stress disorder, and alcohol and drug abuse.

Medication Use: The variables included were: number of oral antidiabetic medication classes and insulin use as identified by national drug codes (NDC), presence of polypharmacy identified by use of 6 or more drug classes in the 90 days prior to index date;

Healthcare Seeking Behavior: The variables included were: baseline healthcare utilization factors (number of emergency room visits in 180 days prior to index date, inpatient hospitalization, number of outpatient visits measured in quartiles);

Community Level Access to Care: The variables included were: whether county of residence had a community mental health clinic (CMHC) and federally qualified health clinic (FQHC), whether county of residence designated as urban area and Health Professional Shortage area (HPSA) for mental health;

Community Resources: The variables included were: median income in the county, whether percent below poverty level and percent with college education greater than national average based on US census estimates [45, 46].

Additionally, the model also controlled for year of observation. 


\section{Statistical Analysis}

\section{Unadjusted Analyses:}

Descriptive analysis using frequency and mean and standard errors were conducted. Chi-square tests were used to examine unadjusted association between baseline characteristics and types of coexisting chronic physical conditions. Unadjusted associations between types of coexisting chronic physical conditions and newly-diagnosed depression were also examined with chi-square tests. Chi-square tests were also used to examine the associations between types of coexisting chronic physical conditions and newly-diagnosed depression for each group of the categorical independent variables.

\section{Adjusted Analyses:}

Multivariable complementary log-log regressions were performed used to examine the risk of newly-diagnosed depression associated with types of coexisting conditions. As the authors intended to estimate "risk" of newly-diagnosed depression instead of "odds", the complementary $\log -\log$ regressions were used instead of logistic regression, which are in general used to estimate the associations between independent variables and binary dependent variable. It has been shown that odds ratio often fail to give approximation of risk, especially when disease rates are close to $10 \%$ or more [47]. Complementary log-log regression has been shown to be an alternative statistical model to estimate risk [48] and has been used in previous studies [49]. Regression coefficients from complementary log-log regressions were exponentiated to derive the risk ratios (RR) and adjusted risk ratios (ARR) of newly-diagnosed depression associated with types of coexisting conditions; 95\% upper and lower confidence intervals for RRs and ARRs were calculated by respectively adding and subtracting 1.96 from the exponentiated standard errors from complementary log-log regressions. 
Self-management of conditions are especially difficult when coexisting conditions have widely varying modes of management; individuals with concordant conditions which have similar mode of management as T2DM may therefore may be at lesser risk of developing depression as compared to discordant or dominant conditions. Thus, "concordant conditions only" was used as the reference group in regression analyses. Multiple regression models were examined. In model 1, only types of coexisting conditions were included; in model 2 individual physical make-up and year of observation were included; in model 3 the presence of other psychological factors, medication use and healthcare seeking behavior were added and the final model 4 consisted of all the variables in model 3 and additionally included community level access to care and community resources variables. All analyses were done using Statistical Analytic Software (SAS) version 9.3. 


\section{RESULTS}

\section{Characteristics of the Study Population}

The study population consisted of 59,857 non-elderly, fee-for-service and continuously enrolled Medicaid beneficiaries with T2DM who were depression free during baseline, not dually eligible for Medicare and alive during the study period. Description of the study population is presented in Table 1. Majority of the study population were older adults (53.2\%) between 55 and 64 years and females (62.9\%); $25.2 \%$ were Whites and $30.4 \%$ were African Americans and $44.4 \%$ were other races. Majority of the counties of residence were metro areas (86.9\%); $84.4 \%$ of counties had shortage of mental health professionals and $53.3 \%$ of counties did not have a CMHC.

\section{Study Population Characteristics by Types of Coexisting Chronic Physical Conditions}

Table 1 also presents a description of study population by types of coexisting conditions. In the study population, $11.2 \%$ had dominant conditions, $35.9 \%$ had concordant conditions only, 17.7\% had discordant conditions only and $35.2 \%$ had both concordant and discordant conditions. All study characteristics differed significantly among the types of coexisting condition subgroups. For example, a greater percentage of individuals with dominant conditions were older i.e.55-64 years (12.8\% vs $6.7 \%$ in $18-44$ years age-group), males (12.7\% vs $10.3 \%$ in females), had presence of other mental health conditions (17.6\% vs $10.1 \%$ with absence of other mental health conditions), inpatient hospitalizations (19.6\% vs $6.7 \%$ in those without) and higher number of outpatient visits $\left(19.6 \%\right.$ in $4^{\text {th }}$ vs $4.6 \%$ in $1^{\text {st }}$ quartile). 


\section{Unadjusted Associations between Types of Coexisting Chronic Physical Conditions and Newly-Diagnosed Depression}

Overall, $10 \%$ of Medicaid enrollees with T2DM had newly-diagnosed depression during the follow up year. The rate of newly-diagnosed depression was highest among those with dominant conditions (13.1\%) followed by both concordant and discordant conditions (12.2\%), discordant conditions only (8.7\%) and concordant conditions only (7.5\%). Chi-square analysis showed that newly-diagnosed depression rates significantly differed by types of coexisting chronic physical conditions $(\mathrm{P}<0.001)$. This significant association between types of coexisting chronic physical conditions and newly-diagnosed depression was observed among all categories of independent variables.

\section{Relationships between Types of Coexisting Chronic Physical Conditions and Newly-}

\section{Diagnosed Depression}

Model 1, adjusted for the type of coexisting chronic physical conditions. In this model, as compared to Medicaid beneficiaries with concordant conditions, those with dominant conditions were at $81 \%$ higher risk (Adjusted Risk Ratio (ARR): 1.81; 95\% Confidence Interval (CI): 1.67,1.96; $\mathrm{p}<0.001)$, those with both concordant and discordant conditions were at $67 \%$ higher risk (AOR: 1.67; 95\% CI: 1.57, 1.77; $\mathrm{p}<0.001$ ) and those with discordant conditions were at 18\% (ARR: 1.18; 95\% CI: 1.09,1.28; p<0.001) higher risk to develop newly-diagnosed depression.

This statistically significant higher risk of newly-diagnosed depression among those with dominant, both discordant and concordant conditions and discordant conditions only as compared to those with concordant conditions only persisted in Model 2 which controlled for individual physical make-up such as age, sex and race and year of observation. In model 3, the 
significant association between discordant conditions and newly-diagnosed depression disappeared, after additionally controlling for other psychological factors, medication use and healthcare seeking behaviors such as inpatient, outpatient and emergency room visits. After controlling for county level variables such as community level access to care and community resources variables in the fully adjusted model 4 , those with dominant conditions were at were at 17\% higher risk (ARR: 1.17; 95\% CI: 1.07, 1.20; $\mathrm{p}=0.0006$ ) and those with both concordant and discordant conditions were found to be at 30\% higher risk (ARR: 1.30; 95\% CI: 1.22, 1.39; $\mathrm{p}<.0001)$ to develop newly-diagnosed depression as compared to those with concordant conditions only. Results of multivariable complementary log-log regression analyses are presented in Table 3. Appendix B presents ARRs and 95\% CIs of all independent variables controlled in the models. 


\section{DISCUSSION}

This study examined whether the risk of newly-diagnosed depression differed by types of coexisting chronic physical conditions among non-elderly fee-for-service Medicaid beneficiaries with T2DM. Overall, $10 \%$ of the study population had newly-diagnosed depression. Even after controlling for all baseline variables, it was found that Medicaid beneficiaries with T2DM and dominant conditions and also those with both concordant and discordant conditions were at higher risk of developing newly-diagnosed depression as compared to those with concordant conditions only.

The dominant conditions in our study included diseases such as cancer, Alzheimer's and Parkinson's diseases. These diseases carry very high burden in terms of mortality and disability $[50,51]$. Therefore, it is not surprising that individuals with T2DM and dominant conditions were at higher risk of developing newly-diagnosed depression, as compared to those with concordant conditions only. Coexisting life threatening illnesses such as cancer can negatively impact self-management behaviors among individuals with T2DM. For example, among those with both cancer and T2DM, Hershey et al., observed that, after 8 weeks of chemotherapy, individuals were able to perform significantly lower number of T2DM self-management behaviors; the most affected behaviors were exercising, ability to eat and drink, and blood sugar monitoring [52]. Neurological conditions such as Parkinson's diseases has been said to cause changes in brain chemistry that may lead to depression [53].

Presence of discordant conditions such as respiratory problems and arthritis are also highly disabling [54]. When such discordant conditions are present along with other concordant conditions, individuals may feel overwhelmed. It has been found that about one third of individuals with both T2DM and arthritis never or rarely exercise owing to reasons such as 
concerns about aggravating arthritis pain, fear about causing further joint damage, uncertainty about which types and amounts of activity are safe for their joints [55]. In general, among individuals with chronic obstructive pulmonary disease (COPD), dyspnoea i.e. breathlessness triggered by exercise severely limits physical activity $[56,57]$. However, regular exercise is essential for management of concordant conditions such as hypertension and cardiovascular conditions. Thus coexistence of such conditions may be overwhelming and may cause poor selfmanagement of conditions. It has been observed that among adults with diabetes, higher number of macro- and micro-vascular and non-diabetes related coexisting conditions are associated with poor self-management [58]. Inability to manage conditions effectively may lead to feelings of frustration and guilt, subsequently leading to depression.

\section{Implications}

The study results have important clinical and policy implications. As a part of the Patient Protection and Affordable Care Act (PPACA) passed in 2010, many states have expanded their Medicaid coverage to individuals at or below $138 \%$ of the Federal poverty level. Therefore, a greater number of individuals who were previously uninsured are now covered by state Medicaid programs. Illinois and New York are among the twenty-eight states that decided to expand their Medicaid programs as of 2014. It is expected that with the new expansion, 571,000 individuals in Illinois and 631,000 individuals in New York may gain access to Medicaid [59]. An analysis of the 2009 Medical Expenditure Panel Survey data showed that 5\% of uninsured individuals at or below $138 \%$ of Federal poverty level, who may become newly eligible for Medicaid as a result of the expansion had diabetes and $64 \%$ of these individuals had at least one coexisting chronic physical condition [33]. Based on the results of the current study, it may be speculated that these 
individuals, if they already do not have prevalent depression, may be at high risk of developing newly-diagnosed depression.

Challenges of coexisting depression among those with T2DM are many and include increased risk of complications, increased health care expenditures and also increased risk of mortality [32]. Individuals with T2DM are considered to be at higher risk for newly-diagnosed depression [21]. Coexisting chronic physical conditions which may aggravate this risk should be closely monitored and screened for depression. Given the higher risk of newly-diagnosed depression among individuals with dominant and among those with both concordant and discordant conditions, such individuals should be regularly screened for depression in primary care settings. The U.S. Preventive Services Task Force (USPSTF) recommends depression screening in all adults and the recommendation has also been supported by American College of Preventive Medicine. Depression screening among adults with T2DM has also been recommended by American Diabetes Association [60]. However, these recommendation only applies to clinical settings with that have systems in place with care management and staff assistance for depression care as in absence of such systems, appropriate diagnosis, treatment plan and follow-up of depression may not be feasible [61], thereby rendering screening efforts ineffective.

The PPACA promotes such integrated care through patient centered medical homes (PCMH) and Accountable Care Organizations (ACO). The PCMH model promotes team based approaches to patient care. Such settings can form teams with primary care physicians, mental health care specialists and a care managers so as to regularly screen for depression among individuals with varying types of coexisting chronic physical conditions and do appropriate follow-up with care managers in case a diagnosis for depression is made [62]. Performance 
measures for Accountable Care Organizations also include screening for depression [63].

Provision of such novel health care systems may go a long way in timely diagnosis of depression among individuals with T2DM and coexisting chronic physical conditions.

\section{Strengths and Limitations}

The study has many advantages. The use of administrative claims data allowed for the identification of T2DM, depression and a wide array of physical and other mental conditions and additionally, helped in establishing a 365 day depression free period. Unlike previous studies which have used a 120 day depression free period to define newly-diagnosed depression [64], using a greater look-back period of 365 days may minimize the misclassification of an episodic manifestation of chronic depression (where depression symptoms last for two or more years) as newly-diagnosed depression. The availability of healthcare encounters across a variety of providers enabled us to control for a comprehensive set of variables such polypharmacy, antidiabetes medication use, and propensity to seek healthcare. The use of a theoretical framework enabled us to summarize the forty-four coexisting conditions into four categories. To the best of the authors' knowledge this is the first study to assess the risk of newly-diagnosed depression among different types of coexisting chronic physical conditions in adults with T2DM, using such framework.

However, this study also had some limitations. Depression was identified using diagnosis codes. Therefore there is a potential for underestimating newly-diagnosed depression rates owing to undiagnosed depression and under-coding of depression. However, the use of diagnoses codes that are recommended by HEDIS and extensively used by health plans in order to identify depression claims and the ability to identify physician/psychologist diagnoses of depression, offer a particularly attractive alternative to the substantial cost and complications associated with 
prospective surveys supplemented with medical record data. T2DM and other coexisting conditions were also identified using diagnosis codes in medical claims. Incomplete or erroneous records submitted by healthcare providers, limited clinical detail in the ICD-9-CM codes and inaccurate demographic information might limit the accuracy of administrative data. As the study population was restricted to only fee-for-service Medicaid beneficiaries, the results are not generalizable to beneficiaries who are enrolled in managed care. In addition, we used only three states and results cannot be extrapolated to other states due to differing Medicaid eligibility and enrollment criteria. 


\section{CONCLUSION}

One in ten Medicaid beneficiaries with T2DM developed depression. The risk of developing depression among those with T2DM varied by types of coexisting conditions; Medicaid beneficiaries with T2DM and dominant conditions and those with both concordant and discordant conditions were at higher risk of developing newly-diagnosed depression. This research built on previous research studies that indicated that individuals with T2DM may have high risk of depression and successfully identified non-elderly adults with T2DM who may even be at a higher risk of developing depression. The study findings suggest that to reduce this increased risk of newly-diagnosed depression, those with dominant conditions and combinations of concordant and discordant conditions need to be under constant surveillance. However, in order for surveillance to be effective, in case such individuals get diagnosed with depression, appropriate treatment and follow-up must be provided. Future research may examine specific dominant conditions and combinations of concordant and discordant conditions that may result in high risk of newly diagnosed depression among adults with T2DM. 


\section{REFERENCES:}

1. World Health Organization. Fact Sheet: Diabetes. Available at: http://www.who.int/mediacentre/factsheets/fs312/en/.

2. Preskorn, S.H., Antidepressant drug selection: criteria and options. J Clin Psychiatry, 1994. 55 Suppl A: p. 6-22; discussion 23-4, 98-100.

3. Shaw, J.E., R.A. Sicree, and P.Z. Zimmet, Global estimates of the prevalence of diabetes for 2010 and 2030. Diabetes Res Clin Pract, 2010. 87(1): p. 4-14.

4. $\quad$ Ferrari, A.J., et al., Burden of depressive disorders by country, sex, age, and year: findings from the global burden of disease study 2010. PLoS Med, 2013. 10(11): p. e1001547.

5. Hasin, D.S., et al., Epidemiology of major depressive disorder: results from the National Epidemiologic Survey on Alcoholism and Related Conditions. Arch Gen Psychiatry, 2005. 62(10): p. 1097-106.

6. Pies, R.W., The Bereavement Exclusion and DSM-5: An Update and Commentary. Innov Clin Neurosci, 2014. 11(7-8): p. 19-22.

7. $\quad$ Bisschop, M.I., et al., The longitudinal relation between chronic diseases and depression in older persons in the community: the Longitudinal Aging Study Amsterdam. J Clin Epidemiol, 2004. 57(2): p. 187-94.

8. de Jonge, P., et al., Prevalent and incident depression in community-dwelling elderly persons with diabetes mellitus: results from the ZARADEMP project. Diabetologia, 2006.

49(11): p. 2627-33.

9. $\quad$ Engum, A., The role of depression and anxiety in onset of diabetes in a large populationbased study. J Psychosom Res, 2007. 62(1): p. 31-8.

10. Aarts, S., et al., Diabetes mellitus type II as a risk factor for depression: a lower than expected risk in a general practice setting. Eur J Epidemiol, 2009. 24(10): p. 641-8.

11. Kim, J.M., et al., Vascular risk factors and incident late-life depression in a Korean population. Br J Psychiatry, 2006. 189: p. 26-30.

12. Brown, L.C., et al., Type 2 diabetes does not increase risk of depression. CMAJ, 2006. 175(1): p. 42-6.

13. Chen, P.C., et al., Population-based cohort analyses of the bidirectional relationship between type 2 diabetes and depression. Diabetes Care, 2013. 36(2): p. 376-82.

14. Palinkas, L.A., P.P. Lee, and E. Barrett-Connor, A prospective study of Type 2 diabetes and depressive symptoms in the elderly: the Rancho Bernardo Study. Diabet Med, 2004. 21(11): p. 1185-91.

15. Polsky, D., et al., Long-term risk for depressive symptoms after a medical diagnosis. Arch Intern Med, 2005. 165(11): p. 1260-6.

16. Maraldi, C., et al., Diabetes mellitus, glycemic control, and incident depressive symptoms among 70- to 79-year-old persons: the health, aging, and body composition study. Arch Intern Med, 2007. 167(11): p. 1137-44.

17. Golden, S.H., et al., Examining a bidirectional association between depressive symptoms and diabetes. JAMA, 2008. 299(23): p. 2751-9.

18. Pan, A., et al., Bidirectional association between depression and type 2 diabetes mellitus in women. Arch Intern Med, 2010. 170(21): p. 1884-91.

19. O'Connor, P.J., et al., Does diabetes double the risk of depression? Ann Fam Med, 2009. 7(4): p. 328-35. 
20. Nefs, G., et al., The course of depressive symptoms in primary care patients with type 2 diabetes: results from the Diabetes, Depression, Type D Personality Zuidoost-Brabant (DiaDDZoB) Study. Diabetologia, 2012. 55(3): p. 608-16.

21. Mezuk, B., et al., Depression and type 2 diabetes over the lifespan: a meta-analysis. Diabetes Care, 2008. 31(12): p. 2383-90.

22. Nouwen, A., et al., Type 2 diabetes mellitus as a risk factor for the onset of depression: $a$ systematic review and meta-analysis. Diabetologia, 2010. 53(12): p. 2480-6.

23. HEDIS. 2008. Health Plan and Employer Data Information Set.Washington, DC:

National Committee for Quality Assurance.

24. Clak, D.A. and A.T. Beck, Scientific foundations of cognitive theory and therapy of depression. 1999: John Wiley \& Sons.

25. Lustman, P.J. and G.W. Harper, Nonpsychiatric physicians' identification and treatment of depression in patients with diabetes. Compr Psychiatry, 1987. 28(1): p. 22-7.

26. Clarke, J.L. and D.C. Meiris, Building bridges: integrative solutions for managing complex comorbid conditions. Am J Med Qual, 2007. 22(2 Suppl): p. 5S-16S.

27. Piette, J.D. and E.A. Kerr, The impact of comorbid chronic conditions on diabetes care. Diabetes Care, 2006. 29(3): p. 725-31.

28. Pentakota, S.R., et al., Does diabetes care differ by type of chronic comorbidity?: An evaluation of the Piette and Kerr framework. Diabetes Care, 2012. 35(6): p. 1285-92.

29. Lepine, J.P. and M. Briley, The increasing burden of depression. Neuropsychiatr Dis Treat, 2011. 7(Suppl 1): p. 3-7.

30. Patten, S.B., Long-term medical conditions and major depression in a Canadian population study at waves 1 and 2. J Affect Disord, 2001. 63(1-3): p. 35-41.

31. Beverly, E.A., et al., Perceived challenges and priorities in co-morbidity management of older patients with Type 2 diabetes. Diabet Med, 2011. 28(7): p. 781-4.

32. Egede, L.E. and C. Ellis, Diabetes and depression: global perspectives. Diabetes Res Clin Pract, 2010. 87(3): p. 302-12.

33. The Kaiser Family Foundation. 2012.The Role of Medicaid for People with Diabetes. Avaiable at: http://kaiserfamilyfoundation.files.wordpress.com/2013/01/8383_d.pdf. Accessed on December 29, 2014

34. Adelmann, P.K., Mental and substance use disorders among Medicaid recipients: prevalence estimates from two national surveys. Adm Policy Ment Health, 2003. 31(2): p. 11129.

35. Pratt, L.A. and D.J. Brody, Depression in the United States household population, 20052006. NCHS Data Brief, 2008(7): p. 1-8.

36. Agency for Healthcare Research and Quality. Statistical Brief \#172. 2011. Conditions

With the Largest Number of Adult Hospital Readmissions by Payer, 2011. Avaiable at: http://www.hcup-us.ahrq.gov/reports/statbriefs/sb172-Conditions-Readmissions-Payer.pdf. Accessed on December 29, 2014.

37. $\quad$ Gilmer, G., T. P., et al., Predictors of health care costs in adults with diabetes. Diabetes Care, 2005. 28(1): p. 59-64.

38. HEDIS® QUALITY MEASURES GUIDE 2013. Avaiable at:

http://www.anthem.com/ca/shared/f0/s0/t0/pw_e192761.pdf? refer=provider. accessed on December 29, 2014. 
39. CMS. Manuscripts and Publications. Available at: http://www.cms.gov/ResearchStatistics-Data-and-Systems/Computer-Data-and-

Systems/Privacy/Manuscripts_Publications.html.

40. Department of Health and Human Services. Area Health Resources Files. Avaiable at: http://ahrf.hrsa.gov/.

41. Stein, B.D., et al., Predictors of adequate depression treatment among Medicaid-enrolled youth. Soc Psychiatry Psychiatr Epidemiol, 2013. 48(5): p. 757-65.

42. $\quad$ Sambamoorthi, U., et al., Diabetes and depression care among medicaid beneficiaries. J Health Care Poor Underserved, 2006. 17(1): p. 141-61.

43. Sambamoorthi, U., et al., Depression treatment patterns among women veterans with cardiovascular conditions or diabetes. World Psychiatry, 2010. 9(3): p. 177-82.

44. Teh, C.F., et al., Predictors of adequate depression treatment among Medicaid-enrolled adults. Health Serv Res, 2010. 45(1): p. 302-15.

45. Census 2000 Brief. Educational Attainment 2000. Avaiable at:

http://www.census.gov/prod/2003pubs/c2kbr-24.pdf. Accessed on Dec 7,2014.

46. Census 2000 Brief. Poverty 1999. Avaiable at:

http://www.census.gov/prod/2003pubs/c2kbr-19.pdf. Accessed on Dec 7,2014.

47. Altman, D.G., J.J. Deeks, and D.L. Sackett, Odds ratios should be avoided when events are common. BMJ: British Medical Journal, 1998. 317(7168): p. 1318.

48. Penman, A.D. and W.D. Johnson, Complementary Log-Log Regression for the Estimation of Covariate-Adjusted Prevalence Ratios in the Analysis of Data from Cross-Sectional Studies. Biometrical Journal, 2009. 51(3): p. 433-442.

49. Bhattacharya, R., C. Shen, and U. Sambamoorthi, Excess risk of chronic physical conditions associated with depression and anxiety. BMC Psychiatry, 2014. 14: p. 10.

50. World Health Organization. Noncommunicable Diseases Country Profiles: United States of America. Avaiable at: http://www.who.int/nmh/countries/usa_en.pdf. Accessed on Jan 2, 2015. 51. World Health Organization. Neurological disorders: public health challenges. Avaiable at: http://www.who.int/mental_health/neurology/neurological_disorders_report_web.pdf. Accessed on January 2, 2015.

52. Hershey, D.S., et al., Perceived impact of cancer treatment on diabetes self-management. Diabetes Educ, 2012. 38(6): p. 779-90.

53. Parkinson's Disease Foundation. Depression. Avaiable at: http://www.pdf.org/en/depression_pd. Accessed on January 2, 2015.

54. Centers for Disease, C. and Prevention, Prevalence and most common causes of disability among adults--United States, 2005. MMWR Morb Mortal Wkly Rep, 2009. 58(16): p. 421-6.

55. Centers for Disease, C. and Prevention, Arthritis as a potential barrier to physical activity among adults with diabetes--United States, 2005 and 2007. MMWR Morb Mortal Wkly Rep, 2008. 57(18): p. 486-9.

56. Calverley, P., Exercise and dyspnoea in COPD. European Respiratory Review, 2006. 15(100): p. 72-79.

57. van der Molen, T., et al., Development, validity and responsiveness of the Clinical COPD Questionnaire. Health Qual Life Outcomes, 2003. 1: p. 13.

58. Kerr, E.A., et al., Beyond comorbidity counts: how do comorbidity type and severity influence diabetes patients' treatment priorities and self-management? J Gen Intern Med, 2007. 22(12): p. 1635-40. 
59. A 50-State Look at Medicaid Expansion. Available at: http://familiesusa.org/product/50state-look-medicaid-expansion. Accessed on January 4, 2014.

60. American Diabetes Association. Depression. Avaiable at: http://www.diabetes.org/livingwith-diabetes/complications/mental-health/depression.html. Accessed January 2, 2014.

61. O'Connor, E.A., et al., Screening for depression in adult patients in primary care settings: a systematic evidence review. Ann Intern Med, 2009. 151(11): p. 793-803.

62. Croghan TW, Brown JD. Integrating Mental Health Treatment Into the Patient Centered Medical Home. (Prepared by Mathematica Policy Research under Contract No.

HHSA290200900019I TO2.) AHRQ Publication No. 10-0084-EF. Rockville, MD: Agency for Healthcare Research and Quality. June 2010. .

63. O'Donnell, A.N., et al., Mental health in ACOs: missed opportunities and low-hanging fruit. Am J Manag Care, 2013. 19(3): p. 180-4.

64. Tiwari, A., et al., Guideline-consistent antidepressant treatment patterns among veterans with diabetes and major depressive disorder. Psychiatr Serv, 2008. 59(10): p. 1139-47. 


\section{TABLES \& APPENDICES}

Table 1: Description of the Study Population by Types of Coexisting Chronic Physical Conditions among Medicaid Beneficiaries with Type 2 Diabetes Mellitus, Multi State Medicaid Claims - 2000 - 2008

\begin{tabular}{|c|c|c|c|c|c|c|c|c|c|c|c|c|}
\hline & & \multicolumn{2}{|c|}{ All } & \multicolumn{2}{|c|}{ Dominant } & \multicolumn{2}{|c|}{$\begin{array}{c}\text { Concordant } \\
\text { Only }\end{array}$} & \multicolumn{2}{|c|}{ Discordant Only } & \multicolumn{2}{|c|}{$\begin{array}{c}\text { Both } \\
\text { Concordant } \\
\text { \& Discordant }\end{array}$} & \multirow[t]{2}{*}{ Sig } \\
\hline & & $\mathbf{N}$ & $\%$ & $\mathbf{N}$ & $\%$ & $\mathbf{N}$ & $\%$ & $\mathbf{N}$ & $\%$ & $\mathbf{N}$ & $\%$ & \\
\hline ALL & & 59,857 & & 6,708 & 11.2 & 21,477 & 35.9 & 10,603 & 17.7 & 21,069 & 35.2 & \\
\hline \multicolumn{13}{|c|}{ Individual Physical Make-up } \\
\hline Age & & & & & & & & & & & & $* * *$ \\
\hline & $18-44$ & 11,676 & 19.5 & 777 & 6.7 & 4,896 & 41.9 & 2,494 & 21.4 & 3,509 & 30.1 & \\
\hline & $45-54$ & 16,314 & 27.3 & 1,847 & 11.3 & 5,496 & 33.7 & 3,184 & 19.5 & 5,787 & 35.5 & \\
\hline & $55-64$ & 31,867 & 53.2 & 4,084 & 12.8 & 11,085 & 34.8 & 4,925 & 15.5 & 11,773 & 36.9 & \\
\hline Sex & & & & & & & & & & & & $* * *$ \\
\hline & Female & 37,635 & 62.9 & 3,892 & 10.3 & 12,625 & 33.5 & 7,231 & 19.2 & 13,887 & 36.9 & \\
\hline & Male & 22,222 & 37.1 & 2,816 & 12.7 & 8,852 & 39.8 & 3,372 & 15.2 & 7,182 & 32.3 & \\
\hline Race & & & & & & & & & & & & $* * *$ \\
\hline & $\begin{array}{l}\text { Whites } \\
\text { African }\end{array}$ & 15,090 & 25.2 & 1,625 & 10.8 & 5,339 & 35.4 & 2,837 & 18.8 & 5,289 & 35.0 & \\
\hline & Americans & 18,200 & 30.4 & 2,088 & 11.5 & 6,223 & 34.2 & 3,337 & 18.3 & 6,552 & 36.0 & \\
\hline & Others & 26,567 & 44.4 & 2,995 & 11.3 & 9,915 & 37.3 & 4,429 & 16.7 & 9,228 & 34.7 & \\
\hline \multicolumn{13}{|c|}{ Psychological Factors } \\
\hline \multicolumn{13}{|c|}{ Other Mental Health } \\
\hline Condi & is & & & & & & & & & & & $* * *$ \\
\hline & Yes & 8,883 & 14.8 & 1,560 & 17.6 & 2,616 & 29.4 & 1,526 & 17.2 & 3,181 & 35.8 & \\
\hline & No & 50,974 & 85.2 & 5,148 & 10.1 & 18,861 & 37.0 & 9,077 & 17.8 & 17,888 & 35.1 & \\
\hline \multicolumn{13}{|c|}{ Medication Use } \\
\hline \multicolumn{8}{|c|}{ Oral Antidiabetic Drugs (OADs) } & & & & & $* * *$ \\
\hline & $1 \mathrm{OAD}$ & 17,996 & 30.1 & 1,890 & 10.5 & 6,288 & 34.9 & 3,511 & 19.5 & 6,307 & 35.0 & \\
\hline & 2 OADs & 14,890 & 24.9 & 1,255 & 8.4 & 5,685 & 38.2 & 2,747 & 18.4 & 5,203 & 34.9 & \\
\hline & $3+$ OADs & 5,292 & 8.8 & 344 & 6.5 & 2,257 & 42.6 & 831 & 15.7 & 1,860 & 35.1 & \\
\hline & No OAD & 21,679 & 36.2 & 3,219 & 14.8 & 7,247 & 33.4 & 3,514 & 16.2 & 7,699 & 35.5 & \\
\hline Insuli & & & & & & & & & & & & $* * *$ \\
\hline & Yes & 18,885 & 31.6 & 2,362 & 12.5 & 7,098 & 37.6 & 1,791 & 9.5 & 7,634 & 40.4 & \\
\hline & No & 40,972 & 68.4 & 4,346 & 10.6 & 14,379 & 35.1 & 8,812 & 21.5 & 13,435 & 32.8 & \\
\hline Polyp & macy & & & & & & & & & & & $* * *$ \\
\hline & Yes & 18,774 & 31.4 & 2,475 & 13.2 & 4,892 & 26.1 & 2,931 & 15.6 & 8,476 & 45.1 & \\
\hline & No & 41,083 & 68.6 & 4,233 & 10.3 & 16,585 & 40.4 & 7,672 & 18.7 & 12,593 & 30.7 & \\
\hline
\end{tabular}


Table 1: Description of the Study Population by Types of Coexisting Chronic Physical Conditions among Medicaid Beneficiaries with Type 2 Diabetes Mellitus, Multi State Medicaid Claims - 2000 - 2008

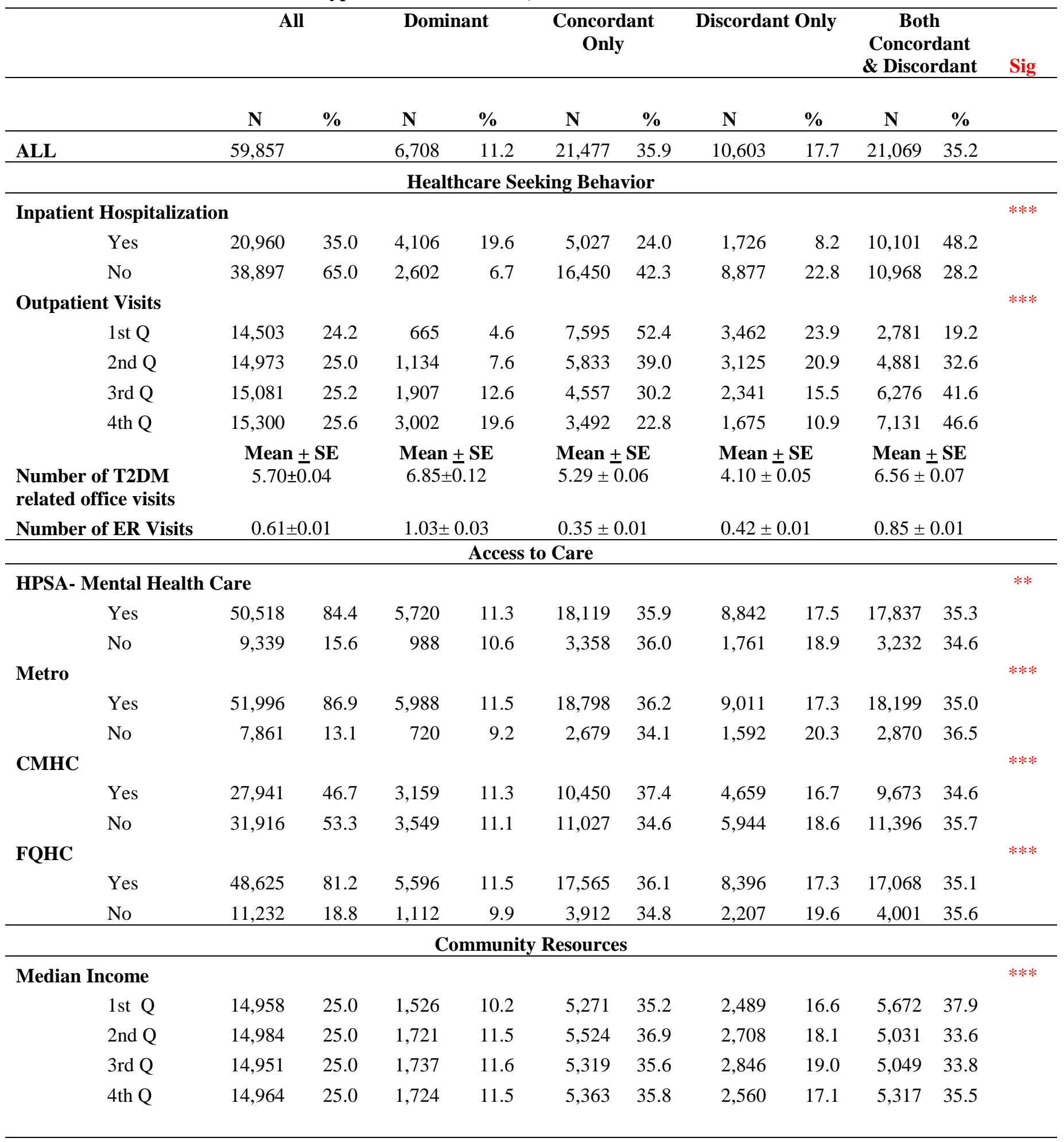


Table 1: Description of the Study Population by Types of Coexisting Chronic Physical Conditions among Medicaid Beneficiaries with Type 2 Diabetes Mellitus, Multi State Medicaid Claims - 2000 - 2008

\begin{tabular}{|c|c|c|c|c|c|c|c|c|c|c|c|c|}
\hline & & \multicolumn{2}{|c|}{ All } & \multicolumn{2}{|c|}{ Dominant } & \multicolumn{2}{|c|}{$\begin{array}{l}\text { Concordant } \\
\text { Only }\end{array}$} & \multicolumn{2}{|c|}{ Discordant Only } & \multicolumn{2}{|c|}{$\begin{array}{c}\text { Both } \\
\text { Concordant } \\
\text { \& Discordant } \\
\end{array}$} & \multirow[t]{2}{*}{ Sig } \\
\hline & & $\mathbf{N}$ & $\%$ & $\mathbf{N}$ & $\%$ & $\mathbf{N}$ & $\%$ & $\mathbf{N}$ & $\%$ & $\mathbf{N}$ & $\%$ & \\
\hline ALL & & 59,857 & & 6,708 & 11.2 & 21,477 & 35.9 & 10,603 & 17.7 & 21,069 & 35.2 & \\
\hline \multicolumn{13}{|c|}{$\begin{array}{l}\% \text { with GT } 4 y r \text { college } \\
\text { education }>16 \%^{t}\end{array}$} \\
\hline & Yes & 47,072 & 78.6 & 5,435 & 11.5 & 16,964 & 36.0 & 8,535 & 18.1 & 16,138 & 34.3 & \\
\hline & No & 12,785 & 21.4 & 1,273 & 10.0 & 4,513 & 35.3 & 2,068 & 16.2 & 4,931 & 38.6 & \\
\hline \multicolumn{12}{|c|}{$\begin{array}{l}\text { \% below poverty level GT } \\
11.1 \%^{\mathrm{H}}\end{array}$} & $* *$ \\
\hline & Yes & 53,092 & 88.7 & 5,968 & 11.2 & 19,060 & 35.9 & 9,307 & 17.5 & 18,757 & 35.3 & \\
\hline & No & 6,765 & 11.3 & 740 & 10.9 & 2,417 & 35.7 & 1,296 & 19.2 & 2,312 & 34.2 & \\
\hline \multirow[t]{4}{*}{ State } & & & & & & & & & & & & $* * *$ \\
\hline & Illinois & 15,674 & 26.2 & 1,659 & 10.6 & 5,525 & 35.2 & 2,895 & 18.5 & 5,595 & 35.7 & \\
\hline & New York & 26,342 & 44.0 & 3,134 & 11.9 & 9,470 & 36.0 & 5,022 & 19.1 & 8,716 & 33.1 & \\
\hline & Texas & 17,841 & 29.8 & 1,915 & 10.7 & 6,482 & 36.3 & 2,686 & 15.1 & 6,758 & 37.9 & \\
\hline
\end{tabular}

Note: Study sample comprised of adults with type 2 diabetes mellitus aged 18-64 years and with at least one coexisting concordant, discordant or dominant chronic physical condition and who were alive, not dually eligible for Medicare and continuously enrolled in fee-for-service Medicaid for at least 24 months( $\mathrm{N}=59,857)$; includes Medicaid data from three states: Illinois, Texas, New York

Asterisks $(*)$ represent significant differences in study population characteristics and coexisting chronic physical conditions categories i.e. Dominant, Concordant Only, Discordant Only and Both Concordant and Discordant, derived from chi-square statistics and univariate ordinary least square regressions.

$* * * \mathrm{P}<.001 ; * * .001 \leq \mathrm{P}<.01 ; * .01 \leq \mathrm{P}<.05$.

$¥ 16 \%$ Cut off was chosen based on 2000 Census Education attainment results \#1 11.1\% Cut off was chosen based on 1999 Census Poverty in 18-64 year results

HPSA: health professional shortage area; CMHC: Community Mental Health Clinic; FQHC: Federally qualified health clinic; GT: Greater Than; Q: Quartile 
Table 2: Percentages of Newly-diagnosed Depression by Types of Coexisting Chronic Physical Conditions among Medicaid Beneficiaries with Type 2 Diabetes Mellitus, Multi-state Medicaid Claims data $-2000-2008$

Percent with Newly-diagnosed depression

Both

\begin{tabular}{|c|c|c|c|c|c|c|c|}
\hline & & ALL & Dominant & $\begin{array}{c}\text { Concordant } \\
\text { Only }\end{array}$ & $\begin{array}{c}\text { Discordant } \\
\text { Only }\end{array}$ & $\begin{array}{l}\text { Concordant } \\
\text { \& Discordant }\end{array}$ & \\
\hline & & $\%$ & $\%$ & $\%$ & $\%$ & $\%$ & Sig \\
\hline ALL & & 10.0 & 13.1 & 7.5 & 8.7 & 12.2 & $* * *$ \\
\hline \multicolumn{8}{|c|}{ Individual Physical Make-up } \\
\hline \multicolumn{8}{|l|}{ Age } \\
\hline & $18-44$ years & 12.6 & 17.1 & 9.8 & 11.2 & 16.7 & $* * *$ \\
\hline & $45-54$ years & 13.0 & 16.5 & 9.3 & 11.0 & 16.5 & $* * *$ \\
\hline & $55-64$ years & 7.5 & 10.8 & 5.6 & 6.0 & 8.7 & $* * *$ \\
\hline \multicolumn{8}{|l|}{ Sex } \\
\hline & Female & 10.6 & 13.3 & 8.3 & 9.4 & 12.6 & $* * *$ \\
\hline & Male & 8.9 & 12.9 & 6.3 & 7.3 & 11.3 & $* * *$ \\
\hline \multicolumn{8}{|l|}{ Race } \\
\hline & $\begin{array}{l}\text { Whites } \\
\text { African }\end{array}$ & 10.6 & 14.2 & 7.8 & 9.6 & 12.8 & $* * *$ \\
\hline & Americans & 9.9 & 13.5 & 7.1 & 8.1 & 12.4 & $* * *$ \\
\hline & Others & 9.7 & 12.3 & 7.6 & 8.6 & 11.6 & $* * *$ \\
\hline \multicolumn{8}{|c|}{ Psychological Factors } \\
\hline \multicolumn{8}{|c|}{ Other Mental Health Conditions } \\
\hline & Yes & 23.4 & 24.8 & 19.8 & 21.2 & 26.8 & $* * *$ \\
\hline & No & 7.6 & 9.6 & 5.8 & 6.6 & 9.6 & **** \\
\hline \multicolumn{8}{|c|}{ Medication Use } \\
\hline \multicolumn{8}{|c|}{ Oral Antidiabetic Drugs (OADs) } \\
\hline & $1 \mathrm{OAD}$ & 9.4 & 11.0 & 7.4 & 8.5 & 11.4 & $* * *$ \\
\hline & 2 OADs & 9.2 & 13.3 & 6.7 & 8.9 & 11.1 & $* * *$ \\
\hline & $3+$ OADs & 9.4 & 11.6 & 7.6 & 8.3 & 11.7 & $* * *$ \\
\hline & No OAD & 11.1 & 14.4 & 8.1 & 8.9 & 13.6 & $* * *$ \\
\hline \multicolumn{8}{|c|}{ Insulin Use } \\
\hline & Yes & 11.1 & 13.9 & 8.2 & 9.0 & 13.4 & $* * *$ \\
\hline & No & 9.5 & 12.7 & 7.1 & 8.7 & 11.4 & $* * *$ \\
\hline \multicolumn{8}{|c|}{ Polypharmacy } \\
\hline & Yes & 11.6 & 14.4 & 8.0 & 11.0 & 13.0 & $* * *$ \\
\hline & No & 9.2 & 12.4 & 7.3 & 7.9 & 11.6 & $* * *$ \\
\hline
\end{tabular}


Table 2: Percentages of Newly-diagnosed Depression by Types of Coexisting Chronic Physical Conditions among Medicaid Beneficiaries with Type 2 Diabetes Mellitus, Multi-state Medicaid Claims data $-2000-2008$

\begin{tabular}{|c|c|c|c|c|c|c|}
\hline & & \multicolumn{5}{|c|}{ Percent with Newly-diagnosed depression } \\
\hline & ALL & Dominant & \multirow{2}{*}{$\begin{array}{c}\begin{array}{c}\text { Concordant } \\
\text { Only }\end{array} \\
\%\end{array}$} & \multirow{2}{*}{$\begin{array}{c}\begin{array}{c}\text { Discordant } \\
\text { Only }\end{array} \\
\% \\
\end{array}$} & $\begin{array}{c}\text { Both } \\
\text { Concordant } \\
\text { \& Discordant }\end{array}$ & \multirow[b]{2}{*}{ Sig } \\
\hline & $\%$ & $\%$ & & & $\%$ & \\
\hline ALL & 10.0 & 13.1 & 7.5 & 8.7 & 12.2 & $* * *$ \\
\hline \multicolumn{7}{|c|}{ Health Seeking Behavior } \\
\hline \multicolumn{7}{|c|}{ Inpatient Hospitalization } \\
\hline Yes & 14.2 & 15.6 & 11.8 & 15.2 & 14.7 & $* * *$ \\
\hline No & 7.7 & 9.3 & 6.2 & 7.5 & 9.8 & $* * *$ \\
\hline \multicolumn{7}{|l|}{ Outpatient Visits } \\
\hline 1 st $\mathrm{Q}$ & 4.6 & 5.9 & 4.2 & 4.2 & 5.7 & $* *$ \\
\hline 2nd Q & 8.2 & 7.4 & 7.8 & 8.1 & 8.9 & \\
\hline 3rd Q & 11.3 & 12.7 & 9.1 & 11.4 & 12.5 & $* * *$ \\
\hline 4th Q & 15.6 & 17.2 & 12.0 & 15.5 & 16.7 & $* * *$ \\
\hline \multicolumn{7}{|c|}{ Access to Care } \\
\hline \multicolumn{7}{|c|}{ HPSA- Mental Health Care } \\
\hline Yes & 9.9 & 13.3 & 7.5 & 8.6 & 11.8 & $* * *$ \\
\hline No & 10.5 & 12.0 & 7.5 & 9.4 & 13.9 & $* * *$ \\
\hline \multicolumn{7}{|l|}{ Metro } \\
\hline Yes & 10.3 & 13.7 & 7.7 & 9.0 & 12.4 & $* * *$ \\
\hline No & 8.1 & 8.6 & 6.0 & 7.2 & 10.4 & $* * *$ \\
\hline \multicolumn{7}{|l|}{ СМHC } \\
\hline Yes & 10.3 & 13.5 & 7.5 & 9.2 & 12.7 & $* * *$ \\
\hline No & 9.7 & 12.8 & 7.5 & 8.4 & 11.7 & $* * *$ \\
\hline \multicolumn{7}{|l|}{ FQHC } \\
\hline Yes & 10.1 & 13.5 & 7.6 & 9.0 & 12.2 & $* * *$ \\
\hline No & 9.3 & 11.1 & 6.7 & 7.9 & 12.0 & $* * *$ \\
\hline \multicolumn{7}{|c|}{ Community Resources } \\
\hline \multicolumn{7}{|l|}{ Median Income } \\
\hline 1 st $\mathrm{Q}$ & 8.3 & 9.8 & 6.2 & 7.4 & 10.1 & $* * *$ \\
\hline 2nd Q & 11.7 & 14.7 & 8.5 & 11.0 & 14.5 & $* * *$ \\
\hline 3rd Q & 11.3 & 15.3 & 8.6 & 9.3 & 13.9 & $* * *$ \\
\hline 4th $\mathrm{Q}$ & 8.6 & 12.3 & 6.5 & 7.0 & 10.4 & $* * *$ \\
\hline \multicolumn{7}{|c|}{$\%$ with GT $4 y r$ college education $>16 \%$} \\
\hline Yes & 10.6 & 14.1 & 7.9 & 9.2 & 12.9 & $* * *$ \\
\hline No & 7.8 & 9.0 & 5.9 & 6.8 & 9.6 & $* * *$ \\
\hline
\end{tabular}

continued

\% below poverty level GT $11.1 \%$ "H 
Table 2: Percentages of Newly-diagnosed Depression by Types of Coexisting Chronic Physical Conditions among Medicaid Beneficiaries with Type 2 Diabetes Mellitus, Multi-state Medicaid Claims data $-2000-2008$

Note: Study population comprised of adults with type 2 diabetes mellitus aged 18-64 years and with at least one coexisting concordant, discordant or dominant chronic physical condition and who were alive, not dually eligible for Medicare and continuously enrolled in fee-for-service Medicaid for at least 24 months( $\mathrm{N}=59,857)$.

Asterisks $(*)$ represent significant differences in developing newly-diagnosed depression among coexisting chronic physical conditions categories i.e. Dominant, Concordant Only, Discordant Only and Both Concordant and Discordant for each of the categorical independent variable subgroups

$* * * \mathrm{P}<.001 ; * * .001 \leq \mathrm{P}<.01 ; * .01 \leq \mathrm{P}<.05$.

$¥ 16 \%$ Cut off was chosen based on 2000 Census Education attainment results H1 11.1\% Cut off was chosen based on 1999 Census Poverty in 18-64 year results

HPSA: health professional shortage area; CMHC: Community Mental Health Clinic; FQHC: Federally qualified health clinic; GT: Greater Than; Q: Quartile 
Table 3: Adjusted Risk Ratios and 95\% Confidence Intervals of Types of Coexisting chronic physical Conditions from Complementary Log-Log Regression on Newly-Diagnosed Depression among Medicaid Beneficiaries with Type 2 Diabetes Mellitus, Multistate Medicaid Claims 2000- 2008 (Reference Group: Concordant only)

\begin{tabular}{|c|c|c|c|c|c|c|c|c|c|c|}
\hline \multirow{2}{*}{$\begin{array}{l}\text { Newly-Diagnosed } \\
\text { Depression }\end{array}$} & \multicolumn{4}{|c|}{ Dominant } & \multicolumn{3}{|c|}{$\begin{array}{c}\text { Discordant Conditions } \\
\text { Only }\end{array}$} & \multicolumn{3}{|c|}{$\begin{array}{c}\text { Both Concordant and } \\
\text { Discordant }\end{array}$} \\
\hline & \multicolumn{10}{|c|}{ Model 1: Adjusted for Types of Coexisting chronic physical Conditions } \\
\hline \multirow{3}{*}{ Yes $($ Ref : No) } & $\mathbf{R R}$ & & $95 \% \mathrm{CI}$ & Sig & $\mathbf{R R}$ & $95 \% \mathrm{CI}$ & Sig & $\mathbf{R R}$ & $95 \% \mathrm{CI}$ & Sig \\
\hline & & 1.81 & {$[1.67,1.96]$} & *** $*$ & 1.18 & {$[1.09,1.28]$} & $* * *$ & 1.67 & {$[1.57,1.77]$} & $* * *$ \\
\hline & ARR & & $95 \%$ CI & Sig & ARR & $95 \% \mathrm{CI}$ & Sig & ARR & $95 \% \mathrm{CI}$ & Sig \\
\hline \multirow{3}{*}{ Yes (Ref: No) } & \multicolumn{10}{|c|}{ Model 2:Adjusted for: Model 1+Individual Physical Make-up } \\
\hline & & 1.92 & {$[1.76,2.08]$} & $* * *$ & 1.09 & {$[1.01,1.19]$} & $*$ & 1.73 & {$[1.63,1.84]$} & $* * *$ \\
\hline & \multicolumn{10}{|c|}{ Model 3: Adjusted for: Model 1+ Model 2+Medication Use and Healthcare Seeking Behavior } \\
\hline \multirow[t]{2}{*}{ Yes (Ref: No) } & & 1.18 & {$[1.08,1.29]$} & $* * *$ & 1.06 & {$[0.98,1.16]$} & & 1.29 & {$[1.21,1.38]$} & $* * *$ \\
\hline & \multicolumn{10}{|c|}{ Model 4: Model 1+ Model 2+Model 3+Community level Access to Care, Community Resources } \\
\hline Yes (Ref: No) & & 1.17 & {$[1.07,1.27]$} & $* * *$ & 1.06 & {$[0.98,1.15]$} & & 1.30 & {$[1.22,1.39]$} & $* * *$ \\
\hline
\end{tabular}

Note: Study sample comprised of adults with type 2 diabetes mellitus aged 18-64 years and with at least one coexisting concordant, discordant or dominant chronic physical condition and who were alive, not dually eligible for Medicare and continuously enrolled in fee-for-service Medicaid for at least 24 months( $\mathrm{N}=59,857)$; includes Medicaid data from three states: Illinois, Texas, New York

Model 1: adjusted for types of coexisting chronic physical conditions.

Model 2 adjusted for gender, age, race/ethnicity and years of observation;

Model 3: Additionally Adjusted for adjusts for presence of other mental health conditions, , number of oral antidiabetic medication classes and insulin use as identified by NDC codes, presence of polypharmacy number of emergency room visits, inpatient hospitalization, number of total and T2DM related office visits;

Model 4: Additionally adjusts for state of residence, whether county of residence had a community mental health clinic and FQHC, CMHC, metro and HPSA-mental health status of county, median income in the county, whether percent below poverty level and percent with college education greater than national average

Asterisks $(*)$ indicate statistical significances are based on complementary log-log regressions with concordant conditions as reference group for types of coexisting chronic physical conditions. $* * * \mathrm{P}<0.001 ; * * .001 \leq \mathrm{P}<.01$; $* .01 \leq \mathrm{P}<.05$.

T2DM: type 2 diabetes mellitus; HPSA: health professional shortage area; CMHC: Community Mental Health Clinic; FQHC: Federally qualified health clinic; Ref: Reference Group 
Appendix A : ICD-9-CM Codes for Identifying Types of Coexisting chronic physical Conditions

\begin{tabular}{|c|c|}
\hline Conditions & ICD-9-CM Codes \\
\hline $\begin{array}{l}\text { Concordant conditions } \\
\text { Coronary Artery Disease }\end{array}$ & $\begin{array}{l}410,4100,4101,4102,4103,4104,4105,4106,4107,4108,4109,411,4110, \\
4111,4118,41181,41189,412,413,4130,4131,4139,414,4140,41400,414 \\
01,41402,41403,41404,41405,4141,41410,41411,41419,4148,4149\end{array}$ \\
\hline Congestive Heart Failure & $40201,40211,40291,40401,40411,40491,428,4280,4281,4289$ \\
\hline Arrhythmia & $423,4230,4231,4232,4238,4239,42731$ \\
\hline Stroke & $\begin{array}{l}\text { 431,43301,43311,43321,43331,43381,43391,43401,43411,43491,435, } \\
4350,4351,4352,4353,4358,4359,438,4380,4381,43811,43812,4382,43 \\
83,4384,4385,43850,4385,43852,43853,4388,43881, \\
43882,43889,4389\end{array}$ \\
\hline Peripheral Vascular Disease & $\begin{array}{l}2507,4402,44020,44021,44022,44023,44024,44029,4408,4409,4422,4 \\
423,443,4430,4431,4438,44381,44389,4439,44422,44481\end{array}$ \\
\hline Peripheral Vascular Disease-gangrene & 7854 \\
\hline $\begin{array}{l}\text { Renal } \\
\text { Chronic Renal Failure } \\
\text { Chronic Pathophysiology }\end{array}$ & $\begin{array}{l}40311,40391,40412,40413,40492,40493,585,586,587, \\
2741,27410,27411,27419, \\
40310,40390,40410,40411,40490,40491, \\
581,5810,5811,5812,5813,5818,5819, \\
582,5820,5821,5822,5824,5828,58281,58289,5829,583,5830,5831,583 \\
2,5834,5836, \\
5837,5838,58381,58389,5839,5900,59000,59001,5936,5939, \\
75312,75313,75314\end{array}$ \\
\hline Diabetic Nephropathy & $2504,25040,25041,25042,25043$ \\
\hline Acute Renal Failure and Disease & $\begin{array}{l}40300,40301,40400,40401,40402,40403,40501,4533,584,5845,5846,5 \\
847,5848,5849, \\
580,5800,5804,5808,58081,58089,5809,5901,59010,59011,5902,5903, \\
5908,59080, \\
59081,59381,866,8660,86600,86601,86602,86603,8661,86610,86611, \\
86612,86613\end{array}$ \\
\hline $\begin{array}{l}\text { Retinopathy } \\
\text { (excludes advanced retinopathy, blindness) }\end{array}$ & $3620,36201,25050,25051,25052,25053$ \\
\hline Ulcer & $700,68110,68111,6827,7071,73076,73077$ \\
\hline $\begin{array}{l}\text { Other Diabetes Related Complications } \\
\text { Uncontrolled Diabetes } \\
\text { Short Term Diabetes } \\
\text { Discordant Conditions }\end{array}$ & $\begin{array}{l}25002,25003,25010,25011,25012,25013,25020,25021,25022,25023,25 \\
030,25031, \\
25032,25033\end{array}$ \\
\hline $\begin{array}{l}\text { Gastro Intestinal Tract Related Disorders: } \\
\text { GERD/Esophagitis } \\
\text { Peptic ulcers } \\
\text { Inflammatory Bowel Disease } \\
\text { Diverticulitis } \\
\text { Gall Bladder disease and stone } \\
\text { Viral hepatitis }\end{array}$ & $\begin{array}{l}5301,5302,5303,53081,531,532,533,534,555,556,56211,56213,574 \\
575,576,070\end{array}$ \\
\hline $\begin{array}{l}\text { Chronic Obstructive Pulmonary Disease } \\
\text { Gout }\end{array}$ & 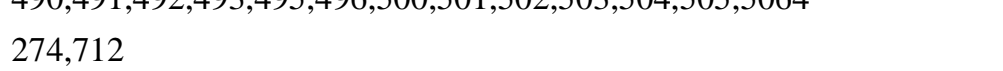 \\
\hline Hip problem & $\begin{array}{l}71905,71915,71925,71935,71945,71955,71965,71975,71985,71995,72 \\
65,73314,73315,73342,820\end{array}$ \\
\hline
\end{tabular}


Appendix A : ICD-9-CM Codes for Identifying Types of Coexisting chronic physical Conditions

\begin{tabular}{|c|c|}
\hline Conditions & ICD-9-CM Codes \\
\hline Low back pain & $\begin{array}{l}\text { 720,7213,72142,72210,72252,72273,72283, } \\
72293,72402,7242,7243,7244,7245,7246,7247,7248,7249\end{array}$ \\
\hline Osteoarthritis & 715 \\
\hline Other Arthritis & 716 \\
\hline Rheumatoid arthritis & 714 \\
\hline Connective tissue rheumatological disease & $7100,7101,7104,725$ \\
\hline Blindness Single Eye & $3696,3697,3698,3699$ \\
\hline \multicolumn{2}{|l|}{ Dominant Conditions } \\
\hline End Stage Renal Disease & E8791,V51,V56,V560,V5631,V5632,V568 \\
\hline End Stage Liver Disease & $5722,5723,5724,5728,4560,4561,4562,45620,45621,571$ \\
\hline Blindness Both Eyes/ Advanced Retinopathy & $36202,3690,3691,3692,3693,3694$ \\
\hline Cancer & $\begin{array}{l}140,141,142,143,144,145,146,147,148,149,150,151,152,153,154,155,1 \\
56,157,158,159,160,161,162,163,164,165,166,167,168,169,170,171,17 \\
2,174,175,176,177,178,179,180,181,182,183,184,186,187,188,189,190 \\
, 191,192,193,194,195,196,197,198,199,200,201,202,203,204,205,206, \\
207,208\end{array}$ \\
\hline Pre-dementia Cognitive Impairment & $294,2941,29283,2949,33183,78093,438,3330,3334,3315$ \\
\hline Dementia and Related Conditions & $\begin{array}{l}2900,29010,29011,29012,29013,2902,29021,2903,29040,29041,29042 \\
\text {,29043,2912, } \\
29410,29411,2948,3310,3311,3312,3317,33182,33189,3319,3320,046 \\
1,0463,0941, \\
29282,3109\end{array}$ \\
\hline Multiple Sclerosis & 340 \\
\hline Hemiplegia Hemiparesis and Paraplegia & 342,3441 \\
\hline Parkinson's Disease & 332 \\
\hline Muscular dystrophy & 359 \\
\hline Spinal cord injury & $\begin{array}{l}80600,80601,80602,80603,80604,80605,80606,80607,80608,80609,80 \\
61,9520, \\
34400,34401,34402,34403,34404,34409\end{array}$ \\
\hline Epilepsy & 345 \\
\hline Gastropareis & 5363 \\
\hline AIDS & 042 \\
\hline
\end{tabular}


Appendix B: Adjusted Risk Ratios and 95\% Confidence Intervals from Complementary Log-Log Regression on Newly-diagnosed Depression among Medicaid Beneficiaries with Type 2 Diabetes Mellitus, Multistate Medicaid Claims 2000-2007

\begin{tabular}{|c|c|c|c|c|c|c|c|c|c|c|c|c|}
\hline & \multicolumn{3}{|c|}{ Model 1} & \multicolumn{3}{|c|}{ Model 2} & \multicolumn{3}{|c|}{ Model 3} & \multicolumn{3}{|c|}{ Model 4} \\
\hline & ARR & $95 \% \mathrm{CI}$ & Sig & ARR & $95 \% \mathrm{CI}$ & Sig & ARR & $95 \% \mathrm{CI}$ & Sig & ARR & $95 \% \mathrm{CI}$ & Sig \\
\hline \multicolumn{13}{|c|}{ Biological Risk Factors } \\
\hline \multicolumn{13}{|c|}{ Types of Coexisting chronic physical conditions } \\
\hline Dominant & 1.81 & {$[1.67,1.96]$} & $* * *$ & 1.92 & {$[1.76,2.08]$} & $* * *$ & 1.18 & {$[1.08,1.29]$} & $* * *$ & 1.17 & {$[1.07,1.27]$} & $* * *$ \\
\hline $\begin{array}{l}\text { Concordant } \\
\text { Conditions Only }\end{array}$ & \multicolumn{12}{|c|}{ Reference } \\
\hline $\begin{array}{l}\text { Discordant } \\
\text { Conditions Only }\end{array}$ & 1.18 & {$[1.09,1.28]$} & $* * *$ & 1.09 & {$[1.01,1.19]$} & $*$ & 1.06 & {$[0.98,1.16]$} & & 1.06 & {$[0.98,1.15]$} & \\
\hline Both & 1.67 & {$[1.57,1.77]$} & $* * *$ & 1.73 & {$[1.63,1.84]$} & $* * *$ & 1.29 & {$[1.21,1.38]$} & $* * *$ & 1.30 & {$[1.22,1.39]$} & $* * *$ \\
\hline \multicolumn{13}{|c|}{ Individual Physical Make-up } \\
\hline \multicolumn{13}{|l|}{ Age } \\
\hline 18-44 years & \multicolumn{12}{|c|}{ Reference } \\
\hline $45-54$ years & & & & 0.99 & {$[0.92,1.06]$} & & 1.00 & {$[0.93,1.07]$} & & 1.01 & {$[0.95,1.08]$} & $* * *$ \\
\hline 55-64 years & & & & 0.52 & {$[0.48,0.55]$} & $* * *$ & 0.57 & {$[0.54,0.61]$} & $* * *$ & 0.59 & {$[0.55,0.63]$} & \\
\hline Sex & & & & & & & & & & & & $* * *$ \\
\hline Female & & & & 1.24 & {$[1.18,1.31]$} & $* * *$ & 1.41 & {$[1.33,1.49]$} & $* * *$ & 1.42 & {$[1.34,1.50]$} & \\
\hline Male & \multicolumn{12}{|c|}{ Reference } \\
\hline Race & & & & & & & & & & & & $* * *$ \\
\hline Whites & & & & 1.15 & {$[1.07,1.23]$} & $* * *$ & 1.24 & {$[1.15,1.32]$} & $* * *$ & 1.34 & {$[1.25,1.44]$} & \\
\hline African Americans & \multicolumn{12}{|c|}{ Reference } \\
\hline Others & & & & 1.02 & {$[0.96,1.08]$} & & 1.14 & {$[1.07,1.21]$} & $* * *$ & 1.26 & {$[1.18,1.34]$} & \\
\hline \multicolumn{13}{|c|}{ Psychological Factors } \\
\hline \multicolumn{13}{|l|}{$\begin{array}{l}\text { Other Mental Health } \\
\text { Conditions }\end{array}$} \\
\hline Yes & & & & & & & 2.60 & {$[2.45,2.76]$} & $* * *$ & 2.51 & {$[2.36,2.66]$} & $* * *$ \\
\hline No & & & & & & Refere & ace & & & & & \\
\hline
\end{tabular}


Appendix B: Adjusted Risk Ratios and 95\% Confidence Intervals from Complementary Log-Log Regression on Newly-diagnosed Depression among Medicaid Beneficiaries with Type 2 Diabetes Mellitus, Multistate Medicaid Claims 2000-2007

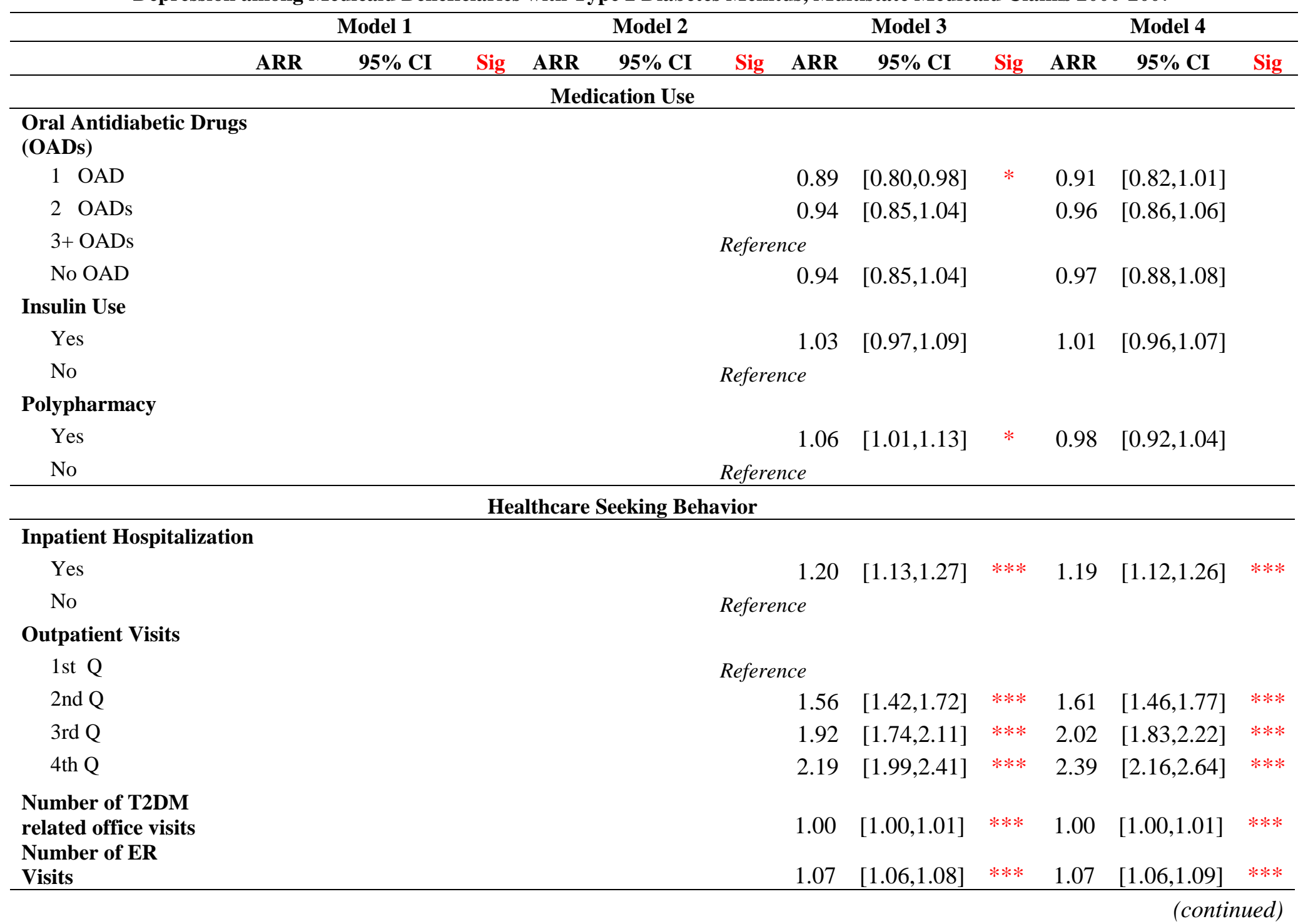

Page 53 of 173 
Appendix B: Adjusted Risk Ratios and 95\% Confidence Intervals from Complementary Log-Log Regression on Newly-diagnosed Depression among Medicaid Beneficiaries with Type 2 Diabetes Mellitus, Multistate Medicaid Claims 2000-2007

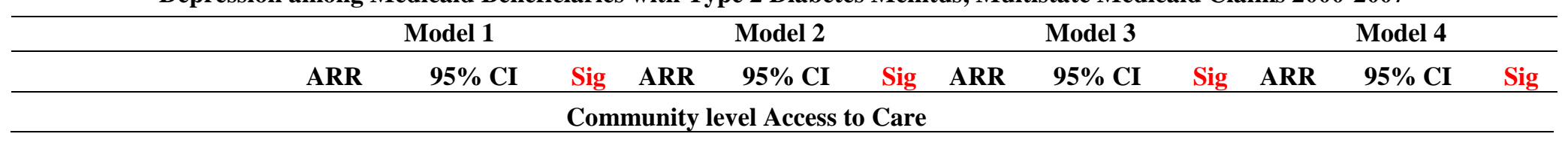

\title{
HPSA- Mental Health Care
}

Yes

No

Metro

Yes

No

CMHC

Yes

No

FQHC

Yes

No

\author{
$0.99 \quad[0.91,1.08]$
}

Reference

$1.04 \quad[0.93,1.17]$

Reference

$0.96 \quad[0.90,1.03]$

Reference

$1.03 \quad[0.94,1.12]$

Reference

Community Resources

$$
\begin{aligned}
& \text { 1st Q } \\
& \text { 2nd Q } \\
& \text { 3rd Q } \\
& \text { 4th Q }
\end{aligned}
$$

Median Income

$\%$ with GT 4yr college

education $>16 \%$ ł

Yes

No

$\begin{array}{lll}0.86 & {[0.74,1.00]} & * \\ 0.91 & {[0.82,1.01]} \\ 0.90 & {[0.81,1.00]} & *\end{array}$

Reference

$0.90 \quad[0.81,1.00]$

$1.07 \quad[0.96,1.20]$

Reference 
Appendix B: Adjusted Risk Ratios and 95\% Confidence Intervals from Complementary Log-Log Regression on Newly-diagnosed Depression among Medicaid Beneficiaries with Type 2 Diabetes Mellitus, Multistate Medicaid Claims 2000-2007

\begin{tabular}{|c|c|c|c|c|c|c|c|c|c|c|c|}
\hline & \multicolumn{2}{|c|}{ Model 1} & \multicolumn{3}{|c|}{ Model 2} & \multicolumn{3}{|c|}{ Model 3} & \multicolumn{3}{|c|}{ Model 4} \\
\hline ARR & $95 \% \mathrm{CI}$ & Sig & ARR & $95 \% \mathrm{CI}$ & Sig & ARR & $95 \% \mathrm{CI}$ & Sig & ARR & $95 \% \mathrm{CI}$ & Sig \\
\hline \multicolumn{12}{|l|}{$\begin{array}{l}\text { \% below poverty level GT } \\
11.1 \%\end{array}$} \\
\hline Yes & & & & & & & & & 1.04 & {$[0.93,1.16]$} & \\
\hline No & \multicolumn{11}{|c|}{ Reference } \\
\hline \multicolumn{12}{|l|}{ State } \\
\hline Illinois & & & & & & & & & 0.81 & {$[0.75,0.88]$} & \\
\hline New York & \multicolumn{11}{|c|}{ Reference } \\
\hline Texas & & & & & & & & & 0.73 & {$[0.66,0.81]$} & \\
\hline \multicolumn{12}{|l|}{ Cohort } \\
\hline 2000-2001 & \multicolumn{11}{|c|}{ Reference } \\
\hline 2001-2002 & & & 1.08 & {$[0.99,1.19]$} & & 1.04 & {$[0.94,1.14]$} & & 1.04 & {$[0.93,1.15]$} & \\
\hline 2002-2003 & & & 1.19 & {$[1.08,1.31]$} & $* * *$ & 1.12 & {$[1.02,1.23]$} & $*$ & 1.09 & {$[0.98,1.23]$} & \\
\hline 2003-2004 & & & 1.01 & {$[0.92,1.11]$} & & 0.92 & {$[0.84,1.02]$} & & 0.91 & {$[0.82,1.01]$} & \\
\hline 2004-2005 & & & 0.83 & {$[0.76,0.92]$} & $* * *$ & 0.79 & {$[0.71,0.86]$} & $* * *$ & 0.78 & {$[0.70,0.86]$} & $* * *$ \\
\hline $2005-2006$ & & & 0.73 & {$[0.67,0.81]$} & $* * *$ & 0.67 & {$[0.61,0.74]$} & $* * *$ & 0.63 & {$[0.56,0.71]$} & $* * *$ \\
\hline 2006-2007 & & & 0.41 & {$[0.37,0.45]$} & $* * *$ & 0.38 & {$[0.34,0.42]$} & $* * *$ & 0.35 & {$[0.31,0.40]$} & $* * *$ \\
\hline
\end{tabular}

Note: Asterisks indicate statistical significances are based on complementary log-log regressions with concordant conditions as reference group for independent variable. $* * * \mathrm{P}<0.001 ; * * .001 \leq \mathrm{P}<.01 ; * .01 \leq \mathrm{P}<.05$.

T2DM: type 2 diabetes mellitus; HPSA: health professional shortage area; CMHC: Community Mental Health Clinic; FQHC: Federally qualified health clinic 
Page 56 of 173 
CHAPTER 3

Page 57 of 173 


\section{CHAPTER 3: Types of Coexisting Chronic Physical Conditions and Treatment for Depression among Medicaid Beneficiaries with Type 2 Diabetes Mellitus and Newly- diagnosed Depression}

\section{INTRODUCTION}

\section{Coexisting Depression among Individuals with Type 2 Diabetes Mellitus (T2DM)}

Presence of depression among individuals with type 2 Diabetes Mellitus (T2DM) serves as a prototype example of Agency of Healthcare Research and Quality (AHRQ)'s definition of a complex patient who has "two or more chronic conditions where each condition may influence the care of the other condition(s) through limitations of life expectancy, interactions between drug therapies, difficulties in establishing adequate care coordination, and/or direct contraindications to therapy for one condition by other conditions " [1]. Depression has considerable adverse impact on both psychological well-being and T2DM related outcomes. Coexisting depression among individuals with type 2 diabetes mellitus (T2DM) is significantly associated with adverse T2DM related outcomes such as poor processes of care (glycated hemoglobin i.e. A1C testing, LDL testing and eye examination)[2], non-adherence to the T2DM treatment regimens [3-8] and poor foot care [7, 9-11].

\section{Treatment for Depression among Individuals with T2DM}

Randomized clinical trials have been conducted to examine the effect of antidepressant treatment for depression among individuals with T2DM and coexisting depression. These trials have evaluated reduction in depressive symptoms, remission from depression, and T2DM-realted outcomes. As compared to placebo, eight weeks of treatment with nortriptyline resulted in a significant reduction in depressive symptoms (reduction in Beck's Depression Inventory score: 10.2 vs $-5.8, \mathrm{p}=.03$ ). However, among 28 individuals with coexisting T2DM and depression, randomized double-blind placebo-controlled trial found that glycemic control worsened among 
individuals who were treated with nortriptyline [12]. As compared to placebo, eight weeks of treatment with fluoxetine resulted in reduction in depressive symptoms (reduction in BDI score: -14.0 vs. $-8.8, \mathrm{P}=0.03$ ) among sixty patients with diabetes (did not distinguish between type 1 and type 2). This trial did not report any significant differences in glycemic control between placebo and treatment groups [13]. Another randomized placebo-controlled double-blind maintenance treatment trial that included individuals who responded to open label sertraline treatment, found that as compared to placebo, individuals who continued treatment with sertraline had significantly lower risk of depression recurrence (hazard ratio $=0.51 ; 95 \% \mathrm{CI}$, 0.31-0.85; $\mathrm{P}=.02$ ); again no significant differences were observed for glycemic control [14]. Double blind treatment with paroxetine as compared to placebo, conducted among 49 mildly depressed individuals, recruited from an outpatient setting in Finland, showed significant improvements in quality of life (mean difference in SF-36 score: 11.0 points, $p=0.039$ ) as well as glycemic control (mean difference in HbA1C: $0.59 \%$ units, $p=0.018$ ) at 3 months, but the effect did not persist at 6 months [15]. Treatment of depression among 51 individuals with 10 weeks of cognitive behavioral therapy has been shown to result in more remission rates as compared to control group that received no specific antidepressant treatment (85\% in CBT group vs $27 \%$ in control group); HbA1C levels at 6 month after follow-up though significantly lower in CBT group (9.5\% compared with $10.9 \%$ in control; $\mathrm{P}=0.03$ ), was above the widely accepted 9\% cut-off for uncontrolled HbA1C in both treatment and control groups [16]. Therefore, it may be concluded that depression treatment may be beneficial for favorable depression related outcomes; however, evidence for T2DM related outcomes is inconclusive. These findings suggest that among individuals with coexisting T2DM and depression, depression treatment may be favored to reduce depressive symptoms. 


\section{Depression Treatment Patterns among Individuals with T2DM}

Only a few studies have examined rates of depression treatment in real-world practice settings among individuals with T2DM and coexisting depression. Findings from the handful of studies indicate that depression among individuals with T2DM is undertreated; for example, Katon and colleagues found that in a study that included both elderly and non-elderly participants (mean age 59 years), only $43 \%$ of with diagnosed depression and T2DM received treatment with 1 or more antidepressants and only $6.7 \%$ received at least 4 psychotherapy sessions during a follow-up period of 12-months [17]. A study among veteran clinic users examined guideline consistent depression treatment within 6 months after diagnosis. This study found that only $51 \%$ of individuals with T2DM and newly-diagnosed depression received any antidepressant treatment [18]. Another study, also among veterans, but restricted to women with newly-diagnosed depression and T2DM, coronary artery disease or hypertension found that $54 \%$ of those with T2DM only used antidepressants alone and 28\% used psychotherapy [19].

Some studies have compared depression treatment rates among individuals with and without T2DM. The rates of depression treatment for those with T2DM were parsed from these studies and are reported below. A study using Medicaid claims data files from Alabama, Georgia, New Jersey, and Wisconsin reported that among Medicaid enrollees with depression, those with diabetes had higher rates of antidepressant treatment (78\%). A nationally representative study of non-institutionalized US population aged 16 years or older that examined the association of treatment modality for depression and burden of comorbid chronic conditions using the Medical Expenditure Panel Survey data, reported that while among individuals with diabetes (did not distinguish between type 1 and type 2) and depression, 21\% did not receive any treatment, about 58\% were treated with antidepressants alone and $14 \%$ were treated with both 
antidepressants and psychotherapy [20]. Another study that used the MedStat MarketScan Commercial Claims and Encounters and Medicare Supplemental and Coordination of Benefits databases, found that $93 \%$ of individuals with diabetes (did not distinguish between type 1 and type 2) and depression were using antidepressants, selective serotonin reuptake inhibitors being the most prescribed class (64\%) [21]. Therefore, among individuals with T2DM rates of depression treatment with antidepressants in the reviewed literature varied from a moderate rate of $43 \%$ to as high as $93 \%$; only one study examined depression treatment rates with psychotherapy.

\section{Depression Treatment among Individuals with Coexisting Chronic Physical Conditions}

Although not specific to T2DM, treatment for depression has been shown to "compete", often unsuccessfully among individuals with multiple coexisting chronic physical conditions [22, 23]. For example, one study found that among 240 individuals with 5 or more depressive symptoms, number of other chronic coexisting chronic physical conditions was inversely associated with the attention given to depression in a physician office visit [22]. Using a sample of 92 participants from the same study, it was found that only $17 \%$ of individuals completed depression treatment and additionally, severity of other coexisting chronic physical conditions reduced the odds of initiating depression treatment even among patients who were enthusiastic about depression treatment [24]. Therefore, these studies indicate that those with multiple chronic conditions are less likely to be treated for depression suggesting that depression treatment among those with T2DM and coexisting chronic physical conditions may be undertreated. However, other existing studies have indicate that competing demands do not affect depression treatment among individuals with multiple coexisting chronic conditions [25-28]. For example, a study using 2007 National Health and Nutrition Examination Survey from found that 
individuals with multiple chronic physical conditions were as likely as those with single condition to report depression treatment [28]. With such conflicting results in previous studies, depression treatment rates among those with T2DM and other multiple coexisting chronic physical conditions remains to be explored.

\section{Depression Treatment among Individuals with T2DM and Coexisting Chronic Physical}

\section{conditions}

A handful of studies already mentioned above, have indirectly examined the association between types of coexisting chronic physical conditions and depression treatment patterns among individuals with T2DM. However, coexisting chronic physical conditions in these studies were measured using either comorbidity indices $[18,29]$ or number of coexisting conditions [19]. Two of these studies found that the number of coexisting chronic condition variable or the comorbidity index variable was not significantly associated with receipt of depression treatment $[18,19]$. On the contrary, in the study among Medicaid beneficiaries with T2DM by Sambamoorthi et al.,[29] it was reported that individuals with higher burden from coexisting conditions, were more likely to receive antidepressants compared to those with no burden from coexisting conditions. However, since these studies used comorbidity indices or number of chronic condition variable in their analyses, there is an existing knowledge gap regarding how different types of coexisting conditions may effect depression treatment among individuals with T2DM and coexisting chronic physical conditions.

\section{Adequate Depression Treatment}

Guidelines for depression treatment suggest that during the first four months after depression diagnosis, those with depression should be treated for at least 84 days with antidepressants [1, 30] and/or at least 8 sessions of psychotherapy [31]. Adequate depression 
treatment either with antidepressants or psychotherapy has both been found to be associated with improvements in depression symptoms [32], whereas, early discontinuation of depression treatment, specifically with antidepressants has been found to be associated with high risk of relapse $[33,34]$.

Among individuals who received depression treatment, the duration of treatment has been found to be often inadequate. An analysis of the National Comorbidity Survey Replication reported that in the US, among individuals with major depression and who received treatment, only $10 \%$ got adequate treatment [35]. Another study that analyzed Medicaid managed care data from a large Mid-Atlantic state in the US, found that, $30 \%$ of enrollees received adequate antidepressant treatment, $63 \%$ of enrollees received adequate psychotherapy defined as four or more psychotherapy visits.

\section{Adequate Depression Treatment among Individuals with T2DM and Coexisting Chronic}

\section{Physical conditions}

Similarly, individuals with T2DM and coexisting depression do not receive adequate depression treatment. For example, Katon et al found that among individuals with diabetes and depression, who were enrolled in a private health maintenance organization, $31 \%$ received adequate antidepressant treatment and only $6.7 \%$ received four or more sessions of psychotherapy [17]. The study by Tiwari et al., among veterans with T2DM and newlydiagnosed depression also found that only $31 \%$ received adequate antidepressant treatment [18]. This study controlled for Charlson Comorbidity Index (CCI) in their logistic regression model for examining the odds of receiving adequate depression treatment. The odds ratio for the CCI variable was not statistically significant, suggesting that coexisting conditions was not associated with receipt of adequate depression treatment among veterans with T2DM. Treatment with 
psychotherapy was not examined in this study. As depression treatment often competes with treatment for other conditions, it is possible that even those who start depression treatment may not get the "adequate" treatment as per guidelines for depression treatment. However, to the best of the authors' knowledge no study has examined whether different types of coexisting chronic physical conditions are associated with receiving adequate depression treatment among individuals with T2DM and depression.

\section{Need for the Study}

The existing literature on patterns of depression treatment among individuals with diabetes have focused primarily on antidepressant treatment even though guideline consistent depression treatment includes both psychotherapy and antidepressant treatment [36]. One of these studies has used self-reported information to identify medical conditions and medication use [20]. Additionally, these studies have often controlled for comorbidity indices such as Charlson comorbidity index [18, 29] or for number of comorbidities [19, 20] or have not considered impact of comorbidities at all [37]. By only controlling for comorbidity numbers or indices, one cannot conclude whether the types of coexisting chronic physical conditions are associated receipt of depression treatment with antidepressants only, psychotherapy only, both or receiving no treatment at all. Examining this association is especially important as often treatment for depression is not given priority in presence of multiple coexisting chronic conditions $[22,23]$. Therefore this study aimed to analyze the association between types of coexisting chronic physical conditions and receipt of depression treatment with antidepressants only, psychotherapy only, both or receiving no treatment at all.

Additionally, the current knowledge on receipt of "adequate" depression treatment by types of coexisting chronic physical conditions among individuals with T2DM is very limited. 
Adequate depression treatment is one of the quality measures included in National Committee for Quality Assurance's (NCQA) Healthcare Effectiveness Data and Information Set (HEDIS). These quality measures are used by various health plans including Medicaid and other organizations in the US for the purpose of accreditation, accountability and quality improvement. Therefore, it is essential to examine whether among individuals with T2DM, risk of not receiving adequate depression treatment varies by types of coexisting chronic physical conditions. This study also attempted to analyze the association between types of coexisting chronic physical conditions and receipt of adequate depression treatment among Medicaid enrollees with T2DM and newly-diagnosed depression.

The study population included Medicaid enrollees. Medicaid is a major payer for nonelderly adults in the US and it accounts for $68 \%$ of federal spending [38]. Traditionally, Medicaid has been a primary source of health insurance for those with low socioeconomic status, which incidentally is a risk factor for developing depression [39]. Additionally, it has been estimated that, $27 \%$ of the African-Americans population in the US receive their health insurance from Medicaid from Medicaid [40]. African-Americans with major depression have been reported to have inadequate depression treatment [41]. Additionally, individuals with diabetes are disproportionately covered by Medicaid, $80 \%$ of Medicaid beneficiaries with diabetes suffer from a coexisting chronic physical condition and $20 \%$ of Medicaid enrollees are diagnosed with depression [27, 28]. Based on these statistics, it can be seen that Medicaid data provides a rich opportunity to conduct research on treatment of depression among individuals with T2DM, depression and coexisting T2DM. 


\section{RESEARCH AIM AND HYPOTHESIS}

Aim 2: Objective 1.1: Examine the association between types of coexisting chronic physical conditions and depression treatment (antidepressants only, psychotherapy only, both

antidepressants and psychotherapy and none), after adjusting for other covariates among nonelderly (18-64 years) fee-for-service Medicaid beneficiaries with T2DM and newly-diagnosed depression,

Aim2: Hypothesis 1.1: As compared to individuals with discordant conditions only, those with concordant conditions would be less likely to receive treatment with antidepressants.

Aim 2: Objective 1.2: Evaluate the association between types of coexisting chronic physical conditions and receipt of adequate depression treatment (yes, no), after adjusting for other covariates among non-elderly (18-64 years) fee-for-service Medicaid beneficiaries with T2DM and newly-diagnosed depression who received either antidepressants or psychotherapy.

Aim2: Hypothesis 1.2: As compared to individuals with discordant conditions only, those with dominant conditions would be less likely to receive adequate depression treatment. 


\section{CONCEPTUAL FRAMEWORK}

The conceptual framework used was based on a modified version of a behavioral model on use of health services, widely known as the Andersen Behavioral Model (ABM) [42]. The ABM posits that utilization of health services varies as a function of 1) each individual's unique predisposition for using services (predisposing factors); 2) the means available to each individual for obtaining services (enabling factors); 3) each individual's level of need; 4) personal health practices; and 5) the external environment. The model is presented below. The behavioral health model is very flexible and was easily adapted to analyze the relationship between types of coexisting chronic physical conditions and depression treatment.

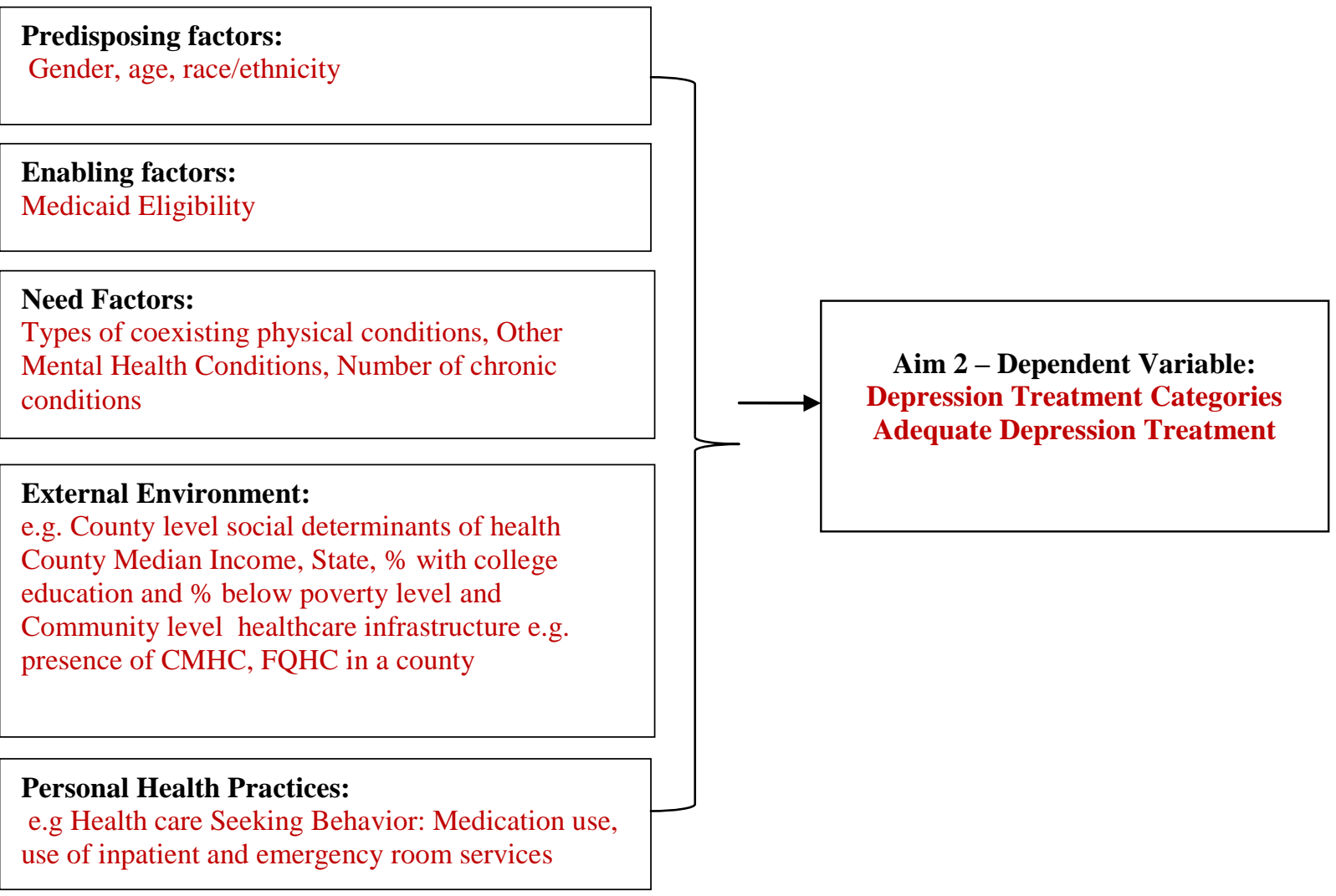




\section{METHODS}

\section{Study Design}

A retrospective cohort study design was used for this study. Individuals with T2DM and newly-diagnosed depression formed the cohort. The date of newly-diagnosed depression was considered as the "index" date. Baseline period was defined as twelve months prior to the index date. All the independent variables were identified during the baseline period. Individuals were followed for a period of 4 months following the index date to examine receipt and depression treatment. This study selected a 4-month follow up period based on the Healthcare Effectiveness Data and Information Set (HEDIS) definition for acute phase of depression treatment [43]. The HEDIS measures antidepressant medication use in the acute phase as 114 days from the date of newly-diagnosed depression i.e. index date [43].

\section{Data Sources}

Medicaid analytic extract (MAX) files:

MAX files are prepared and produced by the Centers for Medicare and Medicaid Services. The MAX files comprise of enrollment ("personal summary"), inpatient claims, other therapy medical claims, and pharmacy claims files of Medicaid beneficiaries. These files were linked by beneficiaries' unique identification number. The person summary file provides information on eligibility, demographics, managed care enrollment, a utilization summary and Medicaid payments. The other files contain information on a variety of factors associated with service provided such as date of service, diagnosis or medication codes associated with the service provided and total amount paid by Medicaid for each service used. This study used 9 years Medicaid data (2000-2008) from three states: New York (NY), Texas (TX), Illinois (IL). It Studies using data that is nationally representative of US population have observed that over the years rates of depression treatment with antidepressants had increased, while rate of treatment 
with psychotherapy decreased in the time period between 1998-2007 [44]. To ensure that enough sample sizes were available for all depression treatment categories, multiple years of claims data were thus combined in this study.

Area Health Resource File (AHRF):

The AHRF is national county-level health resource information provided by the U.S. Department of Health and Human Services, Health Resources and Services Administration [45]. The dataset contains more than 6,000 variables providing county level information on health facilities, health professions, resource scarcity, health status, economic activity, health training programs, and socioeconomic and environmental characteristics [45]. The AHRF file was linked with Medicaid data using the Federal Information Processing Standard (FIPS) county codes. County-level social determinants of health such as median income, percentage below poverty level in a county and county-level healthcare infrastructure such as federally qualified healthcare clinics, health professional shortage area and others (explained in the section on independent variables) were derived from this file.

\section{Identification of the Study Cohort}

\section{Medicaid Beneficiaries with T2DM:}

Individuals with T2DM in a calendar year were identified as those with at least one inpatient visit or two or more physician outpatient visits (which were at least 30 days apart) with a primary or secondary diagnosis of T2DM identified by International Classification of Diagnosis Codes 9th Revision (ICD-9-CM) codes: $250 . x 0$ or $250 . \times 2$. The study population comprised of all individuals with (1) a diagnosis of T2DM (2) no diagnosis for depression and (3) no antidepressant medication use, in the calendar year.

\section{Medicaid Beneficiaries with Newly-diagnosed Depression:}


Medicaid beneficiaries with T2DM and who were depression free were followed in the subsequent calendar year to identify newly-diagnosed depression. Individuals with at least one outpatient visit or an inpatient admission with a primary or secondary diagnosis of depression in the subsequent calendar year were identified as having a newly-diagnosed depression [46]. Depression was identified using ICD9 CM codes: 296.2 (major depressive disorder, single episode), 296.3 (major depressive disorder, recurrent episode), 311 (depression not elsewhere classified), 309.1 (prolonged depressive reaction), 300.4 (neurotic depression) and 298.0 (depressive type psychosis) [19, 29]. The first observed date of outpatient visit or inpatient discharge with diagnosis of depression was the "index date." Therefore, individual classified as having a newly-diagnosed depression had no history of depression diagnosis or antidepressant use for at least a period of 365 days prior to the index date. It has to be noted that previous studies have used a 120 day depression free period to define new episode of depression [18]. However, to minimize misclassification of an episodic manifestation of chronic depression (where depression symptoms last for two or more years) as new episode of depression, the longer look-back period of 365 days was used.

Individuals were excluded from the study cohort if they (1) did not have a diagnosis of at least one coexisting chronic physical conditions (identified by ICD-9-CM codes included in the appendix) during the baseline period (2) did not have continuous fee-for-service Medicaid eligibility during the observation period;(3) were enrolled in Medicare managed care at any point during the observation period; (4) died during the study period; and (5) did not use inpatient or outpatient Medicaid services during the study period.

\section{Dependent Variables}

Depression Treatment during the Acute Phase: 
Depression treatment consisted of pharmacotherapy with antidepressants and psychotherapy. Antidepressant Use: Antidepressants were identified using National Drug Codes (NDC) and included selective serotonin reuptake inhibitors, selective norepinephrine reuptake (inhibitors), tricyclic antidepressants, monoamine oxidase inhibitors, mirtazapine and bupropion. Psychotherapy Use: Psychotherapy was identified using Current Procedural Terminology (CPT) codes. The following psychotherapy types were identified: (i) psychotherapy diagnostic interview $(90801,90802)$ (ii) individual psychotherapy [individual psychotherapy 20-30 min (90804, 90816, 90805, 90817), 45-50 $\min (90806,90818,90807,90819), 75-80 \min (90808$, 90821, 90809, 90822); interactive individual psychotherapy 20-30 min (90810, 90823, 90811, 90824), 45-50 $\min (90812,90826,90813,90827), 75-80 \min (90814,90828,90815,90829)]$ (iii) other psychotherapy [family psychotherapy $(90846,90847,90849)$, group psychotherapy (90853), interactive group psychotherapy(90857)][47].

Depression treatment during the four months of follow-up after newly-diagnosed depression was classified as: (1) Antidepressants only: These individuals received at least one prescription for antidepressant, but no psychotherapy visit during the follow-up.; (2) Psychotherapy only: These individuals received at least one psychotherapy visit, but no prescription for antidepressant drugs during the four months of follow-up. (3) Both antidepressants and psychotherapy: These individuals received a minimum of one prescription for antidepressants and one psychotherapy visit (4) No treatment: These individuals received neither a prescription for antidepressants nor a psychotherapy visit.

Adequate Depression Treatment during the Acute Phase:

Adequate antidepressant treatment: Among individuals who received depression treatment with antidepressants, adequate treatment was defined using the HEDIS criteria. The HEDIS defines 
adequate antidepressant treatment as receiving 84 or more days of prescription for any antidepressant drug during the first 114 days following the index date of depression diagnosis. Based on this definition, individuals with antidepressant prescription during $70 \%$ of the followup days $(84 / 114 * 100=0.71)$ were considered as receiving adequate treatment with antidepressants $[1,30]$

Adequate psychotherapy treatment: Among individuals who received depression treatment with psychotherapy, receipt of adequate treatment was defined as receiving at least 8 sessions (the minimum length of evidence based psychotherapy depression treatment and anxiety disorders) $[31,48]$ during the 120 days of follow-up.

Individuals who received either adequate antidepressant treatment or adequate psychotherapy were defined as having adequate depression treatment. Individuals who did not have adequate antidepressant treatment and who did not have adequate psychotherapy treatment were considered as not having adequate depression treatment.

\section{Independent Variables}

\section{Key Independent Variable: Types of Coexisting Chronic Physical Conditions:}

Based on the theory that coexisting conditions had an impact on the medical care, selfmanagement, and healthcare outcomes of an individual with T2DM, Piette and Kerr developed a framework that classified coexisting conditions among individuals with T2DM into dominant (conditions whose severity eclipses all other conditions' management plans such as metastatic cancer), concordant (conditions that overlap with T2DM in their pathophysiology and management plans such as cardiovascular diseases) or discordant (conditions with unrelated pathophysiology or management plans such as musculoskeletal disorders) [49]. 
Following this framework, it may be assumed that presence of dominant, concordant and discordant conditions among individuals with T2DM and newly-diagnosed depression may affect the choice of depression treatment. Therefore, following a hierarchy defined in a previous study using this framework [50], forty four physical conditions commonly coexisting with T2DM were categorized into: 1) dominant; 2) concordant only, 3) discordant only and 4) both concordant and discordant conditions. Dominant conditions were given priority because such conditions eclipse the management of other health conditions. Concordant and discordant conditions were thus defined only among those without dominant conditions. These conditions were identified using ICD-9-CM codes and are presented in Appendix A.

\section{Other independent variables:}

Other independent variables were chosen based on Andersen's Behavioral Model. These variables were:

Predisposing Factors: The variables included were: demographics (gender, age, race/ethnicity [Whites, African Americans, Hispanics or other races]).

Enabling Factors: The enabling factors were defined using Medicaid eligibility status such as eligibility due to poverty (yes/no), medical needs (yes/no) and waiver (yes/no).

Need Factors: The variables included were clinical characteristics such as presence of other mental health conditions and number of conditions during baseline. The other mental health conditions included bipolar disorder, schizophrenia, post-traumatic stress disorder, and alcohol and drug abuse.

Personal Health Practices: As an individual's propensity to seek treatment may influence receipt of depression treatment, the study controlled for healthcare seeking behavior by controlling for baseline healthcare utilization characteristics such as number of emergency room visits in 180 
days prior to new-onset depression, inpatient hospitalization, number of oral antidiabetic medication classes and insulin use as identified by NDC codes, presence of polypharmacy identified by use of 6 or more drug classes in the 90 days prior to new-onset depression, number of outpatient visits measured in quartiles;

External Environment: External environment variables included state of residence, community level access healthcare infrastructure and community level social determinants of health variables. Community level access healthcare infrastructure variables included presence of community mental health clinic (CMHC) and federally qualified health clinic (FQHC) in a county, whether county of residence was designated as Health Professional Shortage area (HPSA) and density of social workers in a county. Community level social determinants of health variables were defined as external environment variables. The variables included were urban/rural status of a county, median income in the county and whether percent below poverty level and percent with college education in the county were greater than national average based on US census estimates [51, 52].

\section{Statistical Analysis}

\section{Unadjusted Analyses:}

Descriptive analysis was conducted using frequency, mean and standard errors. Chisquare tests were used to examine the unadjusted association between types of coexisting chronic physical conditions and depression treatment. The association between types of coexisting chronic physical conditions and depression treatment were also examined within each category of the independent variables and statistical significance of the associations were assessed using the chi-square tests. Same set of statistical analyses were performed to examine the factors associated with adequate depression treatment. 


\section{Adjusted Analyses:}

Multinomial logistic regression analyses were used to examine adjusted association between types of coexisting conditions and depression treatment after controlling for the other independent variables. Adjusted logistic regression analyses were used to examine adjusted association between types of coexisting conditions and adequate depression treatment after controlling for predisposing, enabling, need, personal health practices and external environment variables. Given that prescription of antidepressants is contraindicated in some concordant and dominant conditions included in the study, in all regression analyses "discordant conditions only" group was used as the reference group. Statistical Analytic Software (SAS 9.3) was used for all analyses. 


\section{RESULTS}

\section{Characteristics of the Study Population}

The study population consisted of 5,837 non-elderly adults Medicaid fee-for-service beneficiaries with T2DM and newly-diagnosed depression who were not enrolled in Medicare and alive during the study period (Table 1). In the study population $67 \%$ were females, $39.7 \%$ were in the 55-64 years, 30.2\% were African-Americans, 36.5\% used polypharmacy and 49.9\% had baseline inpatient hospitalizations; based on county of residence characteristics majority lived in area designated as metro (89.2\%), HPSA for mental health (83.5\%), areas with FQHCs $(82.4 \%)$ and percentage of persons with income below poverty level greater than national average $(88.5 \%)$. Details on the description of the study population are presented in Table 1 .

\section{Study Population Characteristics by Types of Coexisting Chronic Physical Conditions}

The description of the study population by types of coexisting chronic physical condition categories are also presented in Table 1 . In the study population, 14.7\% had dominant conditions; $27.0 \%, 15.4 \%$, and $43.0 \%$ of the study population had concordant only, discordant only, and both concordant and discordant conditions respectively. Significant group differences in types of coexisting conditions were observed for all the other independent variables included in the study. A higher proportion of individuals with dominant conditions were older i.e.55-64 years (18.7\% vs $9 \%$ in 18-44 years age-group), males (18.4\% vs $12.9 \%$ in females), had presence of other mental health conditions $(18.6 \%$ vs $12.6 \%$ with absence of other mental health conditions), inpatient hospitalizations (21.3\% vs $8.2 \%$ in those without) and higher number of outpatient visits $\left(22.9 \%\right.$ in $4^{\text {th }}$ vs $6.1 \%$ in $1^{\text {st }}$ quartile). Table 1 presents the description of the study population characteristics by types of coexisting conditions. 


\section{Depression Treatment: Percentages}

In the study population, $57.3 \%$ received any depression treatment; $27.6 \%$ received treatment with antidepressants only, $18.1 \%$ received treatment with psychotherapy only and $11.6 \%$ received treatment with both antidepressants and psychotherapy, $42.7 \%$ did not receive any treatment.

\section{Unadjusted Associations between Types of Coexisting Chronic Physical Conditions and Depression Treatment Categories}

A significantly lower percentage of individuals with dominant conditions (20.2\%) and concordant conditions only $(27.9 \%)$ had treatment with antidepressants only as compared to those with discordant conditions only (33.1\%); a significantly higher percentage of individuals in dominant conditions $(27.9 \%)$ and concordant conditions only $(17.2 \%)$ received treatment with psychotherapy only as compared to individuals with discordant conditions only (13.4\%). Similar relationships were observed when types of coexisting conditions and depression treatment were examined for each subgroup of the categorical independent variables. For example, among women $20.8 \%$ and $31.2 \%$ in the dominant and concordant conditions only groups were treated with only antidepressants as compared to $34.1 \%$ in discordant conditions only group; $23.4 \%$ and $13.2 \%$ in the dominant conditions and concordant conditions only groups were treated with psychotherapy only as compared to $11.6 \%$ in discordant only group. Percentages of individuals receiving treatment with antidepressants only, psychotherapy only and both antidepressants and psychotherapy for each category of types of coexisting conditions are presented in Table 2; results for no treatment group are not presented in tabular form, but can be furnished on request. Adjusted Associations between Types of Coexisting Chronic Physical Conditions and

\section{Depression Treatment Categories}


The associations observed in unadjusted analyses, remained in adjusted analyses. In adjusted analyses using multinomial logistic regression, the reference group for the dependent variable was "No depression treatment". The reference group for types of coexisting chronic physical conditions was "discordant condition only". The results from the multinomial logistic regression revealed that as compared to individuals with discordant conditions only, those with dominant conditions (AOR: 0.77; 95\% Confidence Interval (CI): 0.59, 0.99; $<<0.05)$ and those with concordant conditions only (Adjusted Odds Ratio (AOR): 0.80; 95\% CI: 0.65, 0.98; $\mathrm{p}<0.05)$ were less likely to receive depression treatment with antidepressants only. In comparison to those with discordant conditions only, treatment of depression with psychotherapy was more likely among individuals with dominant conditions (AOR: 1.61; 95\% CI: 1.21, 1.15; $\mathrm{p}<.01)$ and concordant conditions only: AOR: $1.32 ; 95 \%$ CI: $1.01,1.72 ; \mathrm{p}<.05)$. There was no statistically significant association between types of coexisting chronic physical conditions and receipt of both antidepressant and psychotherapy treatment. The results are presented in Table 3 .

\section{Adequate Depression Treatment}

\section{Descriptive, Unadjusted and Adjusted Associations with Types of Coexisting Chronic Physical}

\section{Conditions:}

Among those who received depression treatment $(\mathrm{N}=3,347), 32.5 \%$ received adequate treatment either with antidepressants or psychotherapy. Bivariate chi-square and multivariate logistic regression analyses did not show any significant associations between types of coexisting conditions and adequate depression treatment.

\section{Other Independent Variables Associated with Adequate Depression Treatment}

From multivariate analyses, it was observed that Whites as compared to African Americans (AOR: 1.44; 95\% CI:1.17, 1.78;p<0.001), 1 OAD (AOR:1.40; 95\% CI:1.05, 1.88; 
$\mathrm{p}<0.05)$ and 2 OADs (AOR:1.45; 95\% CI:1.08, 1.95; $\mathrm{p}<0.05)$ users as compared to users of $3+\mathrm{OADs}$ and those with number of outpatient visits in the fourth quartile as compared to first quartile (AOR:1.79; 95\% CI:1.39, 2.31; p<0.001) were more likely to get adequate depression treatment. (Results not presented in tabular form). 


\section{DISCUSSION}

This study examined the association between types of coexisting conditions and depression treatment, after adjusting for other predisposing, enabling, need, personal health practices and external environment factors, among non-elderly fee-for-service Medicaid Enrollees from multiple states who had T2DM and newly-diagnosed depression.

Depression treatment rates found in this study I somewhat lower than those found in the T2DM population. Less than $40 \%$ of the study population in this received treatment with antidepressants. Though not specific to individuals with T2DM, Marcus et al., reported that among individuals with depression, antidepressant and psychotherapy treatment rates in a nationally representative sample of the US population in 2007 were $75 \%$ and $43 \%$ respectively. The antidepressant treatment rates among individuals with newly-diagnosed depression and T2DM have been found to vary between 93\% [21]to 43\% [17]. Therefore, antidepressant treatment rates in this study were low as compared to other studies.

Nearly, $30 \%$ of the study population received psychotherapy. Psychotherapy treatment rate in nationally representative US sample was $43 \%$ in 2007 [44]. Among those with T2DM, psychotherapy rates have been found to be $28 \%$ in veterans' population. Therefore, psychotherapy treatment rates may be comparable to other studies. However, still $43 \%$ of individuals did not receive any depression treatment. Additionally, only $33 \%$ of those were treated, got adequate depression treatment.

It was observed among individuals with T2DM, those with coexisting conditions that were either related to T2DM pathophysiology or management i.e. concordant conditions only or those with potentially life threatening conditions i.e. dominant conditions were less likely to get prescribed antidepressants and more likely to avail psychotherapy as compared to individuals 
with T2DM and other coexisting conditions that were unrelated to T2DM in terms of pathophysiology or management plans i.e. discordant conditions.

This study found that among individuals with T2DM and newly-diagnosed depression, those with concordant conditions only were less likely to receive treatment with antidepressants only and more likely to receive treatment with psychotherapy only. These results are not surprising given that antidepressants have not yet proven to be highly efficacious for treating depression in presence of concordant conditions such as heart disease [53] [54] [55]. It has been reported that $15 \%-25 \%$ of individuals with heart disease and depression who were initiated on antidepressants, discontinue their treatment because of adverse events [56]. The American Heart Association suggests that if antidepressant treatment is initiated among individuals with heart disease, then these individuals should be closely monitored to ensure medication compliance and to detect and manage any adverse event [57]. Given that presence of T2DM itself is associated with high cardiovascular event risk [58], physicians may avoid prescribing antidepressants to individuals with T2DM and concordant conditions such as heart disease. On the other hand, treating depression with psychotherapy such as cognitive behavioral therapy among individuals with heart disease have been shown to be effective in individuals not getting treat treatment with antidepressants; the ENRICHD trial showed that 12-16 sessions of CBT over 12 weeks help in achieving remission from moderate to severe depression [59].

Individuals with dominant conditions as compared to those with discordant conditions only were more likely to receive depression treatment with only psychotherapy. This may be due to the proven beneficial effects of treating depression with psychotherapy among individuals with dominant conditions[60]. For example, a meta-analysis of six randomized clinical trials found that compared to treatment as usual, psychotherapies such as supportive- 
expressive group therapy, cognitive behavioral therapy, and problem-solving therapy, had significant clinical benefits in reducing depressive symptoms among individuals with advanced cancer [61]. Psychotherapy such as cognitive behavioral therapy has also proven to be beneficial for individuals with end stage renal disease [62]. The effectiveness of antidepressants for depression treatment in patients with terminal illness such as cancer and end stage renal disease remains inconclusive. Additionally, as it may take four to eight weeks to evaluate effectiveness of antidepressants, physicians may be reluctant in initiating treatment with antidepressants in individuals with limited life expectancy [63, 64]. Furthermore, the use of antidepressants for depression treatment in presence of neurological conditions such as dementia [65], Parkinson's [66] and epilepsy [67] (which were also included under definition for dominant conditions) have not been shown to be clinically effective in improving quality of life, and functional and cognitive outcomes and sometimes[68] even in reducing depression symptoms [65].

This study additionally examined the association between types of coexisting conditions and adequate depression treatment among those who were treated for depression after adjusting for predisposing, enabling, need, personal health practices and external environment variables; however, no statistically significant associations between adequate depression treatment and types of coexisting chronic physical conditions were observed. Previous research, although not specific to T2DM, a study using data on veterans also observed similar relationships [69]. This study reported that among veterans with coexisting depression and multiple chronic conditions (cardiovascular/cerebrovascular diseases, arthritis, peptic ulcer, diabetes, respiratory diseases, substance/alcohol abuse and cancer), there were no significant associations between coexisting chronic illnesses and adequate antidepressant treatment [69]. 


\section{Implications}

Individuals with both physical (i.e. T2DM, coexisting chronic physical conditions) and mental health (i.e. depression) often have complex health care needs which may not be fully met in traditionally short (often channeled in to 15 minute time slots) primary care visits [70]. To provide quality care to such individuals, healthcare teams with expertise in providing both physical and mental health care may be more appropriate. Evidence from randomized clinical trials have shown that collaborative care among individuals with depression and T2DM, significantly improved the depressive symptom score and HbA1c level, compared with control conditions [71]. However, even in collaborative care settings, the selection of the healthcare team is important. Meta-regression analysis of randomized clinical trials on collaborative care for depression treatment have shown that characteristics of the intervention provided such as whether case managers were regularly supervised by psychiatrists and the mental health background of case managers had more favorable effect on outcomes [72]. Therefore, such

carefully selected teams with backgrounds in both physical and mental health care delivery may improve quality of depression treatment. The novel healthcare delivery models such as Accountable Care Organizations (ACO) and Patient Centered Medical Home (PCMH) created under the 2010 Patient Protection and Affordable Care Act, provide options for such integrated care models. Future research may focus on quality of depression treatment provided to individuals with both physical and mental health conditions in such settings.

\section{Strengths and limitations}

The current study has many advantages. The use of administrative claims data with dates of services enabled us to follow a cohort of patients with T2DM over time. Medicaid claims also enabled us to capture the healthcare experiences of Medicaid beneficiaries across a variety of 
providers in different locations. The presence of diagnoses enabled identification of T2DM, depression and other physical and mental health conditions. The study used a comprehensive list of independent variables by merging Medicaid claims data with the county-level data from the ARF. A pragmatic practice-based theoretical framework was used to identify the numerous coexisting chronic physical conditions into manageable and clinically meaningful categories. This was the first study to examine whether depression treatment patterns among individuals with T2DM varied by coexisting chronic physical conditions with similar or different management and/or pathophysiology from that of T2DM.

However, the study also has some limitations. As administrative claims data can only identify diseases through diagnosis codes, there is the potential for underestimating depression prevalence or incidence because of under-coding of depression. Identifying depression is one of the more difficult problems in administrative data research and perfection may not be attainable. However, claims data contain physician/psychologist diagnoses of depression and offer a particularly attractive alternative to the substantial cost and complications associated with prospective surveys supplemented with medical record data. Additionally, this study included only filled prescriptions and it is not known whether the antidepressants were actually used. This study did not include alternative forms of treatment for depression. The study included fee-forservice Medicaid beneficiaries enrolled three states, thus results might not be generalizable to other populations. 


\section{CONCLUSIONS}

Types of coexisting conditions were associated with depression treatment categories among non-elderly adult fee-for-service Medicaid beneficiaries; however once treatment was received, whether the individual obtains adequate treatment is not associated with coexisting conditions. The study findings suggest that management for other chronic physical conditions may compete with treatment for depression. Future studies that adjust for physician factors may need to be conducted to explore the management priorities. The findings from this study needs to be replicated in the general population. 


\section{REFERENCES:}

1. National Committee for Quality Assurance. The state of health care quality: 2007. Antidepressant Medication Management. Available at: https://www.ncqa.org/Portals/O/Publications/Resource\%20Library/SOHC/SOHC_07.pdf Accessed July 20, 2014.

2. Frayne, S.M., et al., Disparities in diabetes care: impact of mental illness. Arch Intern Med, 2005. 165(22): p. 2631-8.

3. Cramer, J.A., A systematic review of adherence with medications for diabetes. Diabetes Care, 2004. 27(5): p. 1218-24.

4. DiMatteo, M.R., H.S. Lepper, and T.W. Croghan, Depression is a risk factor for noncompliance with medical treatment: meta-analysis of the effects of anxiety and depression on patient adherence. Arch Intern Med, 2000. 160(14): p. 2101-7.

5. Dirmaier, J., et al., Diabetes in primary care: prospective associations between depression, nonadherence and glycemic control. Psychother Psychosom, 2010. 79(3): p. $172-8$.

6. Gonzalez, J.S., et al., Depression and diabetes treatment nonadherence: a meta-analysis. Diabetes Care, 2008. 31(12): p. 2398-403.

7. Lustman, P.J. and R.E. Clouse, Depression in diabetic patients: the relationship between mood and glycemic control. J Diabetes Complications, 2005. 19(2): p. 113-22.

8. Ciechanowski, P.S., W.J. Katon, and J.E. Russo, Depression and diabetes: impact of depressive symptoms on adherence, function, and costs. Arch Intern Med, 2000. 160(21): p. 3278-85.

9. Ciechanowski, P.S., et al., The relationship of depressive symptoms to symptom reporting, self-care and glucose control in diabetes. Gen Hosp Psychiatry, 2003. 25(4): p. 246-52.

10. Gonzalez, J.S., et al., Symptoms of depression prospectively predict poorer self-care in patients with Type 2 diabetes. Diabet Med, 2008. 25(9): p. 1102-7.

11. McKellar, J.D., K. Humphreys, and J.D. Piette, Depression increases diabetes symptoms by complicating patients' self-care adherence. Diabetes Educ, 2004. 30(3): p. 485-92.

12. Lustman, P.J., et al., Effects of nortriptyline on depression and glycemic control in diabetes: results of a double-blind, placebo-controlled trial. Psychosom Med, 1997. 59(3): p. 241-50.

13. Lustman, P.J., et al., Fluoxetine for depression in diabetes: a randomized double-blind placebo-controlled trial. Diabetes Care, 2000. 23(5): p. 618-23.

14. Lustman, P.J., et al., Sertraline for prevention of depression recurrence in diabetes mellitus: a randomized, double-blind, placebo-controlled trial. Arch Gen Psychiatry, 2006. 63(5): p. 521-9.

15. Paile-Hyvarinen, M., K. Wahlbeck, and J.G. Eriksson, Quality of life and metabolic status in mildly depressed patients with type 2 diabetes treated with paroxetine: a doubleblind randomised placebo controlled 6-month trial. BMC Fam Pract, 2007. 8: p. 34.

16. Lustman, P.J., et al., Cognitive behavior therapy for depression in type 2 diabetes mellitus. A randomized, controlled trial. Ann Intern Med, 1998. 129(8): p. 613-21.

17. Katon, W.J., et al., Quality of depression care in a population-based sample of patients with diabetes and major depression. Med Care, 2004. 42(12): p. 1222-9.

18. Tiwari, A., et al., Guideline-consistent antidepressant treatment patterns among veterans with diabetes and major depressive disorder. Psychiatr Serv, 2008. 59(10): p. 1139-47. 
19. Sambamoorthi, U., et al., Depression treatment patterns among women veterans with cardiovascular conditions or diabetes. World Psychiatry, 2010. 9(3): p. 177-82.

20. Loeb, D.F., et al., Association of treatment modality for depression and burden of comorbid chronic illness in a nationally representative sample in the United States. Gen Hosp Psychiatry, 2012. 34(6): p. 588-97.

21. Le, T.K., et al., Treatment patterns and resource use among patients with comorbid diabetes mellitus and major depressive disorder. J Med Econ, 2011. 14(4): p. 440-7.

22. Rost, K., et al., The role of competing demands in the treatment provided primary care patients with major depression. Arch Fam Med, 2000. 9(2): p. 150-4.

23. Klinkman, M.S., Competing demands in psychosocial care. A model for the identification and treatment of depressive disorders in primary care. Gen Hosp Psychiatry, 1997. 19(2): p. 98-111.

24. Nutting, P.A., et al., Competing demands from physical problems: effect on initiating and completing depression care over 6 months. Arch Fam Med, 2000. 9(10): p. 1059-64.

25. Anderson, K.M., How Far Have We Come in Reducing Health Disparities?: Progress Since 2000: Workshop Summary. 2012: National Academies Press.

26. Kenney, G.M., et al., Opting in to the Medicaid expansion under the ACA: Who are the uninsured adults who could gain health insurance coverage? Urban Institute, 2012. 47.

27. Adelmann, P.K., Mental and substance use disorders among Medicaid recipients: prevalence estimates from two national surveys. Adm Policy Ment Health, 2003. 31(2): p. 111-29.

28. Pratt, L.A. and D.J. Brody, Depression in the United States household population, $2005-$ 2006. NCHS Data Brief, 2008(7): p. 1-8.

29. Sambamoorthi, U., et al., Diabetes and depression care among medicaid beneficiaries. J Health Care Poor Underserved, 2006. 17(1): p. 141-61.

30. Teh, C.F., et al., Predictors of adequate depression treatment among Medicaid-enrolled adults. Health Serv Res, 2010. 45(1): p. 302-15.

31. Foa, E.B., Prolonged exposure therapy: past, present, and future. Depression and anxiety, 2011. 28(12): p. 1043-1047.

32. Duhoux, A., et al., What is the association between quality of treatment for depression and patient outcomes? A cohort study of adults consulting in primary care. J Affect Disord, 2013. 151(1): p. 265-74.

33. Gardarsdottir, H., et al., Duration of antidepressant drug treatment and its influence on risk of relapse/recurrence: immortal and neglected time bias. Am J Epidemiol, 2009. 170(3): p. 280-5.

34. Geddes, J.R., et al., Relapse prevention with antidepressant drug treatment in depressive disorders: a systematic review. Lancet, 2003. 361(9358): p. 653-61.

35. Wang, P.S., et al., Twelve-month use of mental health services in the United States: results from the National Comorbidity Survey Replication. Arch Gen Psychiatry, 2005. 62(6): p. 629-40.

36. National Collaborating Centre for Mental Health. Depression. The treatment and management of depression in adults. London (UK): National Institute for Health and Clinical Excellence (NICE); 2009 Oct. 64 p. (Clinical guideline; no. 90). Avaiable at: http://www.guideline.gov/content.aspx?id=15521. 
37. Manderbacka, K., et al., Diabetes and depression? Secular trends in the use of antidepressants among persons with diabetes in Finland in 1997-2007.

Pharmacoepidemiol Drug Saf, 2011. 20(4): p. 338-43.

38. de Jonge, P., et al., Prevalent and incident depression in community-dwelling elderly persons with diabetes mellitus: results from the ZARADEMP project. Diabetologia, 2006. 49(11): p. 2627-33.

39. Lorant, V., et al., Socioeconomic inequalities in depression: a meta-analysis. Am J Epidemiol, 2003. 157(2): p. 98-112.

40. The Kaiser Family Foundation. Medicaid's Role for Black Americans. 2011.. Available at: http://kaiserfamilyfoundation.files.wordpress.com/2013/01/8188.pdf.

41. Agyemang, A.A., et al., Quality of depression treatment in Black Americans with major depression and comorbid medical illness. Gen Hosp Psychiatry, 2014. 36(4): p. 431-6.

42. Andersen, R.M., Revisiting the behavioral model and access to medical care: does it matter? J Health Soc Behav, 1995. 36(1): p. 1-10.

43. HEDIS. 2008. Health Plan and Employer Data Information Set.Washington, DC: National Committee for Quality Assurance.

44. Marcus, S.C. and M. Olfson, National trends in the treatment for depression from 1998 to 2007. Arch Gen Psychiatry, 2010. 67(12): p. 1265-73.

45. Department of Health and Human Services. Area Health Resources Files. Avaiable at: http://ahrf.hrsa.gov/.

46. Stein, B.D., et al., Predictors of adequate depression treatment among Medicaid-enrolled youth. Soc Psychiatry Psychiatr Epidemiol, 2013. 48(5): p. 757-65.

47. Harpaz-Rotem, I., D. Libby, and R.A. Rosenheck, Psychotherapy use in a privately insured population of patients diagnosed with a mental disorder. Soc Psychiatry Psychiatr Epidemiol, 2012. 47(11): p. 1837-44.

48. Fortney, J.C., et al., The association between rural residence and the use, type, and quality of depression care. J Rural Health, 2010. 26(3): p. 205-13.

49. Piette, J.D. and E.A. Kerr, The impact of comorbid chronic conditions on diabetes care. Diabetes Care, 2006. 29(3): p. 725-31.

50. Pentakota, S.R., et al., Does diabetes care differ by type of chronic comorbidity?: An evaluation of the Piette and Kerr framework. Diabetes Care, 2012. 35(6): p. 1285-92.

51. Census 2000 Brief. Educational Attainment 2000. Avaiable at: http://www.census.gov/prod/2003pubs/c2kbr-24.pdf. Accessed on Dec 7,2014.

52. Census 2000 Brief. Poverty 1999. Avaiable at: http://www.census.gov/prod/2003pubs/c2kbr-19.pdf. Accessed on Dec 7,2014.

53. Glassman, A.H., et al., Sertraline treatment of major depression in patients with acute MI or unstable angina. JAMA, 2002. 288(6): p. 701-9.

54. Monte, S., et al., Antidepressants and cardiovascular outcomes in patients without known cardiovascular risk. Eur J Clin Pharmacol, 2009. 65(11): p. 1131-8.

55. Smoller, J.W., et al., Antidepressant use and risk of incident cardiovascular morbidity and mortality among postmenopausal women in the Women's Health Initiative study. Arch Intern Med, 2009. 169(22): p. 2128-39.

56. Lesperance, F. and N. Frasure-Smith, Depression in patients with cardiac disease: a practical review. J Psychosom Res, 2000. 48(4-5): p. 379-91.

57. Lichtman, J.H., et al., Depression and coronary heart disease: recommendations for screening, referral, and treatment: a science advisory from the American Heart 
Association Prevention Committee of the Council on Cardiovascular Nursing, Council on Clinical Cardiology, Council on Epidemiology and Prevention, and Interdisciplinary Council on Quality of Care and Outcomes Research: endorsed by the American Psychiatric Association. Circulation, 2008. 118(17): p. 1768-75.

58. Grundy, S.M., et al., Diabetes and cardiovascular disease: a statement for healthcare professionals from the American Heart Association. Circulation, 1999. 100(10): p. 113446.

59. Berkman, L.F., et al., Effects of treating depression and low perceived social support on clinical events after myocardial infarction: the Enhancing Recovery in Coronary Heart Disease Patients (ENRICHD) Randomized Trial. JAMA, 2003. 289(23): p. 3106-16.

60. Widera, E.W. and S.D. Block, Managing grief and depression at the end of life. Am Fam Physician, 2012. 86(3): p. 259-64.

61. Akechi, T., et al., Psychotherapy for depression among incurable cancer patients. Cochrane Database Syst Rev, 2008(2): p. CD005537.

62. Duarte, P.S., et al., Cognitive-behavioral group therapy is an effective treatment for major depression in hemodialysis patients. Kidney Int, 2009. 76(4): p. 414-21.

63. Rayner, L., et al., Antidepressants for depression in physically ill people. Cochrane Database Syst Rev, 2010(3): p. CD007503.

64. Rayner, L., et al., Antidepressants for the treatment of depression in palliative care: systematic review and meta-analysis. Palliat Med, 2011. 25(1): p. 36-51.

65. Banerjee, S., et al., Study of the use of antidepressants for depression in dementia: the HTA-SADD trial--a multicentre, randomised, double-blind, placebo-controlled trial of the clinical effectiveness and cost-effectiveness of sertraline and mirtazapine. Health Technol Assess, 2013. 17(7): p. 1-166.

66. Miyasaki, J.M., et al., Practice Parameter: evaluation and treatment of depression, psychosis, and dementia in Parkinson disease (an evidence-based review): report of the Quality Standards Subcommittee of the American Academy of Neurology. Neurology, 2006. 66(7): p. 996-1002.

67. Gilliam, F.G., Diagnosis and treatment of mood disorders in persons with epilepsy. Curr Opin Neurol, 2005. 18(2): p. 129-33.

68. Price, A., et al., Antidepressants for the treatment of depression in neurological disorders: a systematic review and meta-analysis of randomised controlled trials. $\mathrm{J}$ Neurol Neurosurg Psychiatry, 2011. 82(8): p. 914-23.

69. Jordan, N., et al., Association between chronic illness complexity and receipt of evidencebased depression care. Med Care, 2014. 52 Suppl 3: p. S126-31.

70. Margolius, D. and T. Bodenheimer, Transforming primary care: from past practice to the practice of the future. Health Aff (Millwood), 2010. 29(5): p. 779-84.

71. Atlantis, E., P. Fahey, and J. Foster, Collaborative care for comorbid depression and diabetes: a systematic review and meta-analysis. BMJ Open, 2014. 4(4): p. e004706.

72. Gilbody, S., et al., Collaborative care for depression: a cumulative meta-analysis and review of longer-term outcomes. Arch Intern Med, 2006. 166(21): p. 2314-21. 
TABLES \& APPENDICES

Table 1: Description of the Study Population by Types of Coexisting Chronic Physical Conditions Among Medicaid Beneficiaries with Type 2 Diabetes Mellitus and Newly-diagnosed Depression Multi-state Medicaid Claims Database - 2000 - 2008

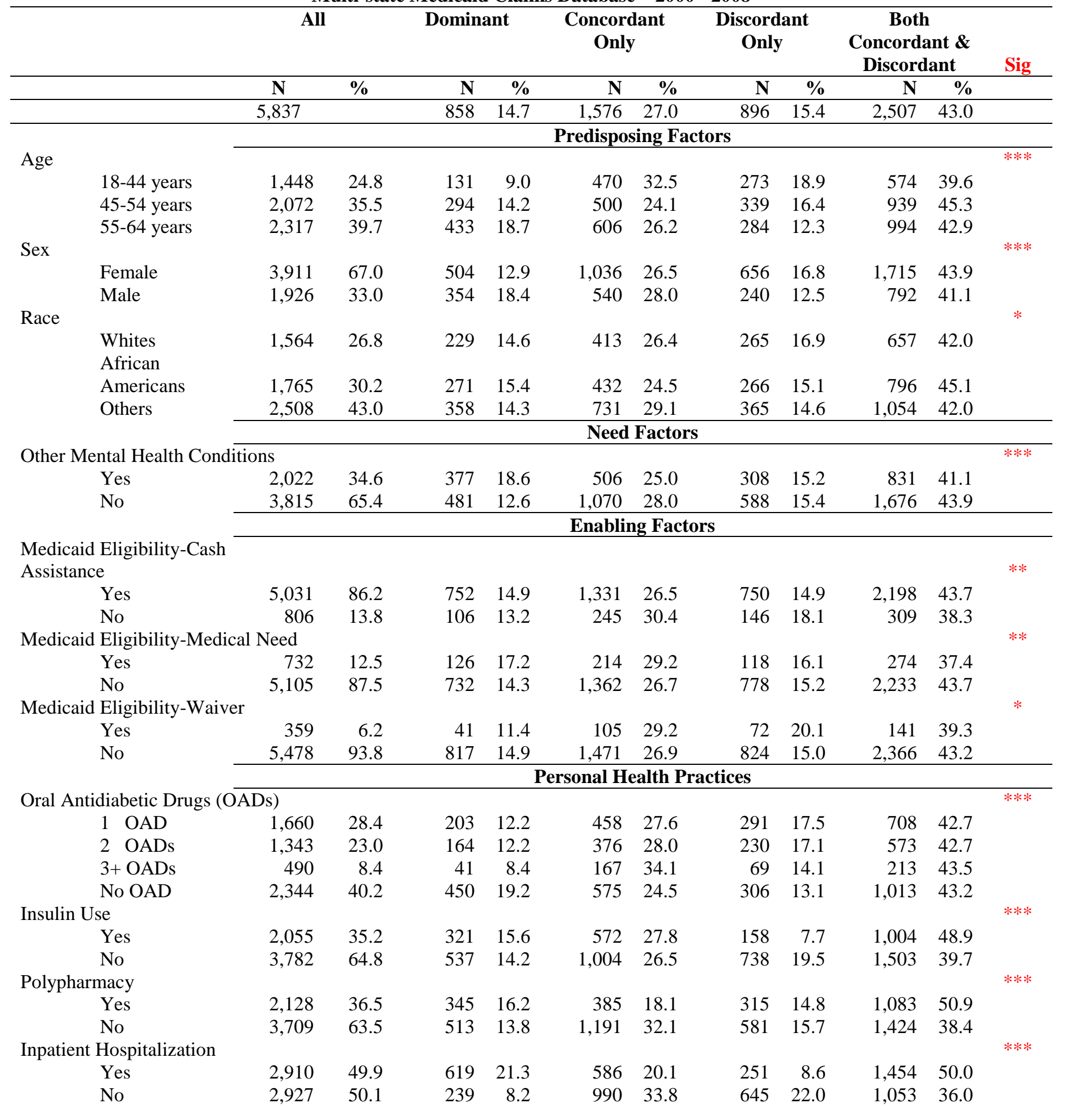


Table 1: Description of the Study Population by Types of Coexisting Chronic Physical Conditions Among Medicaid Beneficiaries with Type 2 Diabetes Mellitus and Newly-diagnosed Depression Multi-state Medicaid Claims Database - 2000 - 2008

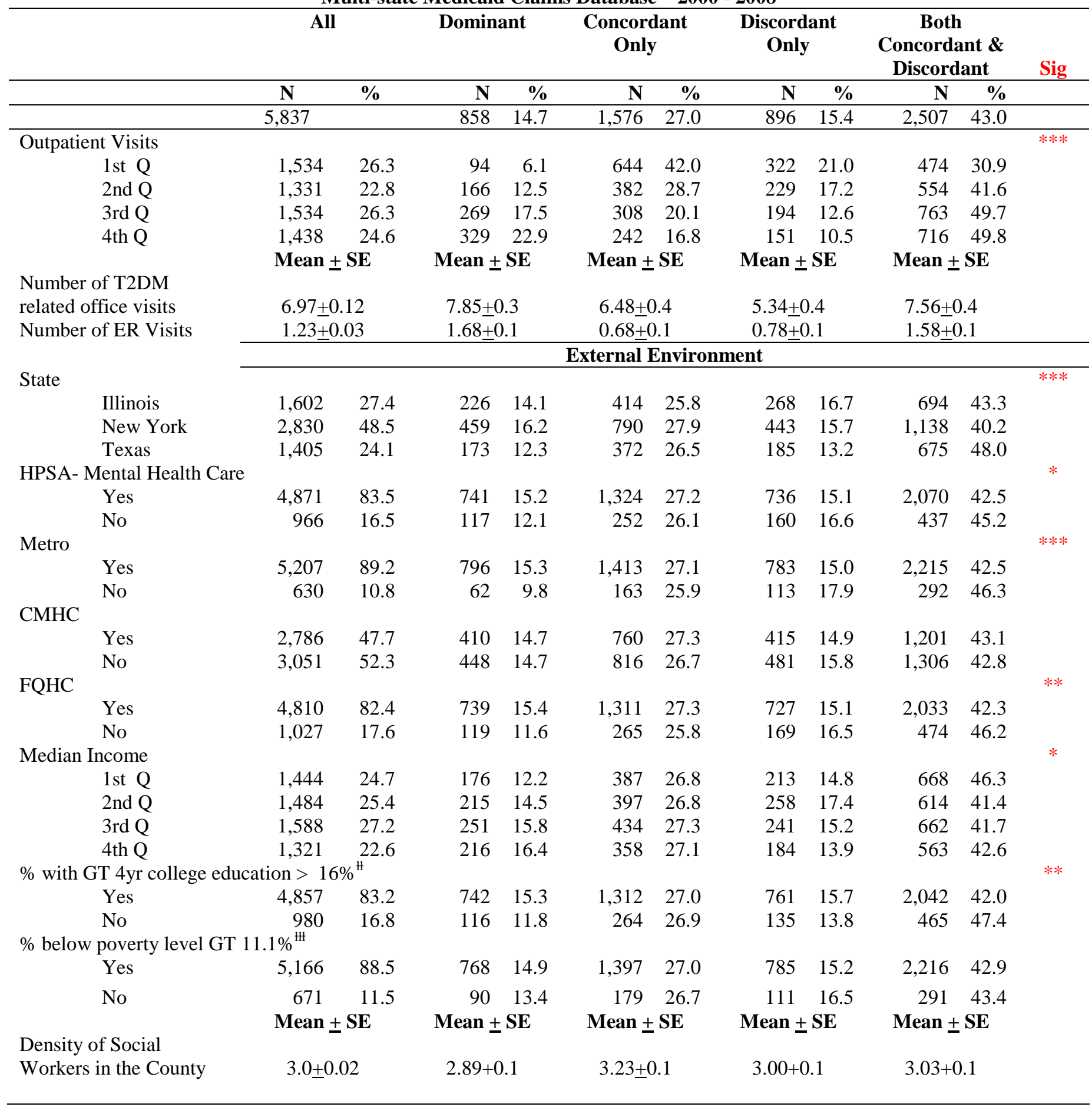

Note: Study sample comprised of individuals with type 2 diabetes mellitus aged 18-64 years and with at least one coexisting dominant, concordant or discordant chronic physical condition and who were alive, not dually eligible for Medicare and continuously enrolled in fee-for-service Medicaid for at least 24 months( $\mathrm{N}=5,837)$; includes Medicaid data from three states: Illinois, Texas, New York 
Asterisks (*) represent significant differences in study population characteristics and coexisting chronic physical conditions categories i.e. Concordant Only, Discordant Only, Both Concordant and Discordant and Dominant, derived from chi-square statistics and univariate ordinary least square regressions (indicated by $¥$ ) $* * * \mathrm{P}<.001 ; * * .001 \leq \mathrm{P}<.01 ; * .01 \leq \mathrm{P}<.05$.

$¥$ Both Concordant and Discordant

\# 16\% Cut off was chosen based on 2000 Census Education attainment results

H1 11.1\% Cut off was chosen based on 1999 Census Poverty levels

AA: African American; Q: Quartile; HPSA: Health Professional Shortage Area; CMHC: Community Mental Health

Clinic; FQHC: Federally qualified health clinic; GT: Greater Than; 
Table 2: Percentages of Depression Treatment by Types of Coexisting Chronic Physical Conditions

Among Medicaid Beneficiaries with Type 2 Diabetes Mellitus and Newly-diagnosed Depression Multi-state Medicaid Claims Database - 2000 - 2008

\begin{tabular}{|c|c|c|c|c|c|c|c|c|c|c|c|c|c|}
\hline & \multirow[b]{2}{*}{ Dominant } & \multicolumn{3}{|c|}{ Antidepressants only } & \multicolumn{3}{|c|}{ Psychotherapy only } & \multicolumn{6}{|c|}{ Both Antidepressants \& Psychotherapy } \\
\hline & & $\begin{array}{c}\text { Concordant } \\
\text { Only } \\
\end{array}$ & $\begin{array}{c}\text { Discordant } \\
\text { Only }\end{array}$ & Both $^{¥}$ & Dominant & $\begin{array}{c}\text { Concordant } \\
\text { Only }\end{array}$ & $\begin{array}{c}\text { Discordant } \\
\text { Only }\end{array}$ & Both $^{¥}$ & Dominant & $\begin{array}{c}\text { Concordant } \\
\text { Only }\end{array}$ & $\begin{array}{c}\text { Discordant } \\
\text { Only }\end{array}$ & Both $¥$ & \\
\hline & $\%$ & $\%$ & $\%$ & $\%$ & $\%$ & $\%$ & $\%$ & $\%$ & $\%$ & $\%$ & $\%$ & $\%$ & Sig \\
\hline \multirow[t]{2}{*}{$\mathbf{A L L}$} & 20.2 & 27.9 & 33.1 & 28.0 & 27.9 & 17.2 & 13.4 & 17.1 & 12.5 & 11.8 & 11.0 & 11.4 & $* * *$ \\
\hline & \multicolumn{12}{|c|}{ Predisposing Factors } & \\
\hline \multicolumn{14}{|l|}{ Age } \\
\hline 18-44 years & 21.4 & 26.4 & 29.3 & 26.3 & 26.0 & 16.6 & 14.7 & 17.9 & 13.0 & 12.6 & 12.5 & 13.2 & \\
\hline $45-54$ years & 22.1 & 25.6 & 33.6 & 27.7 & 24.8 & 20.4 & 13.6 & 16.1 & 12.9 & 13.2 & 10.6 & 12.7 & $* *$ \\
\hline 55-64 years & 18.5 & 30.9 & 36.3 & 29.3 & 30.5 & 15.0 & 12.0 & 17.5 & 12.0 & 10.1 & 10.2 & 9.2 & $* * *$ \\
\hline \multicolumn{14}{|l|}{ Sex } \\
\hline Female & 20.8 & 31.2 & 34.1 & 29.8 & 23.4 & 13.2 & 11.6 & 14.5 & 14.3 & 12.5 & 11.6 & 11.5 & $* * *$ \\
\hline Male & 19.2 & 21.5 & 30.4 & 24.1 & 34.2 & 24.8 & 18.3 & 22.7 & 9.9 & 10.6 & 9.6 & 11.1 & $* * *$ \\
\hline \multicolumn{14}{|l|}{ Race } \\
\hline American & 13.7 & 23.1 & 25.6 & 24.2 & 33.9 & 23.1 & 16.9 & 20.1 & 11.1 & 10 & 13.2 & 10.7 & $* * *$ \\
\hline Others & 24.6 & 27.9 & 36.4 & 29.0 & 24.6 & 12.7 & 11.8 & 15.7 & 9.8 & 12.6 & 9.9 & 10.4 & $* * *$ \\
\hline & \multicolumn{13}{|c|}{ Need Factors } \\
\hline \multicolumn{14}{|c|}{ Other Mental Health Conditions } \\
\hline Yes & 13.5 & 16.8 & 21.8 & 19.9 & 36.6 & 29.1 & 25.3 & 26.4 & 11.9 & 9.3 & 10.1 & 11.9 & $* *$ \\
\hline \multirow[t]{2}{*}{ No } & 25.4 & 33.1 & 39.1 & 32.0 & 21.0 & 11.6 & 7.1 & 12.5 & 12.9 & 13 & 11.6 & 11.2 & $* * *$ \\
\hline & \multicolumn{13}{|c|}{ Enabling Factors } \\
\hline \multicolumn{14}{|c|}{ Medicaid Eligibility-Cash Assistance } \\
\hline Yes & 19.3 & 25.8 & 31.2 & 27.0 & 27.7 & 17.7 & 14.0 & 17.4 & 12.8 & 11.6 & 11.6 & 11.0 & $* * *$ \\
\hline No & 26.4 & 39.2 & 43.2 & 35.0 & 29.2 & 14.3 & 10.3 & 14.6 & 10.4 & 13.1 & 8.2 & 14.6 & $* *$ \\
\hline
\end{tabular}


Table 2: Percentages of Depression Treatment by Types of Coexisting Chronic Physical Conditions

Among Medicaid Beneficiaries with Type 2 Diabetes Mellitus and Newly-diagnosed Depression Multi-state Medicaid Claims Database - 2000 - 2008

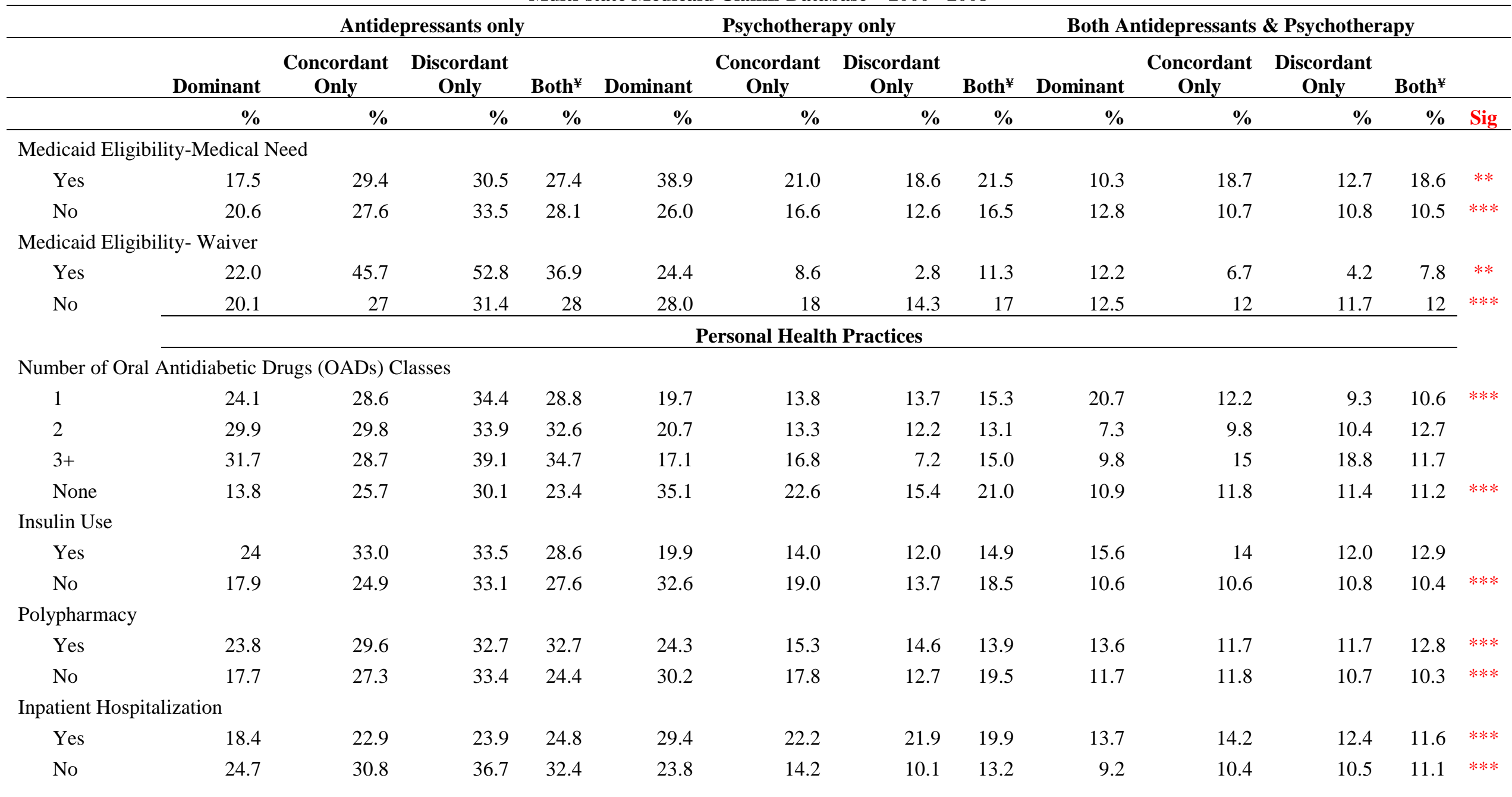


Table 2: Percentages of Depression Treatment by Types of Coexisting Chronic Physical Conditions

Among Medicaid Beneficiaries with Type 2 Diabetes Mellitus and Newly-diagnosed Depression Multi-state Medicaid Claims Database - 2000 - 2008

\begin{tabular}{|c|c|c|c|c|c|c|c|c|c|c|c|c|c|}
\hline & \multicolumn{5}{|c|}{ Antidepressants only } & \multicolumn{2}{|c|}{ Psychotherapy only } & \multicolumn{6}{|c|}{ Both Antidepressants \& Psychotherapy } \\
\hline & Dominant & $\begin{array}{c}\text { Concordant } \\
\text { Only }\end{array}$ & $\begin{array}{c}\text { Discordant } \\
\text { Only }\end{array}$ & Both $^{¥}$ & Dominant & $\begin{array}{c}\text { Concordant } \\
\text { Only }\end{array}$ & $\begin{array}{c}\text { Discordant } \\
\text { Only }\end{array}$ & Both $¥$ & Dominant & $\begin{array}{c}\text { Concordant } \\
\text { Only }\end{array}$ & $\begin{array}{c}\text { Discordant } \\
\text { Only }\end{array}$ & Both $¥$ & \\
\hline & $\%$ & $\%$ & $\%$ & $\%$ & $\%$ & $\%$ & $\%$ & $\%$ & $\%$ & $\%$ & $\%$ & $\%$ & Sig \\
\hline \multicolumn{14}{|c|}{ Outpatient Visits } \\
\hline 1 st $\mathrm{Q}$ & 34 & 32.1 & 36.3 & 34.0 & 17.0 & 11.8 & 11.2 & 12.0 & 9.6 & 12.3 & 12.4 & 11.8 & \\
\hline 2nd Q & 21.7 & 25.9 & 35.4 & 31.9 & 21.7 & 18.3 & 10.9 & 14.1 & 10.8 & 12.3 & 9.6 & 10.6 & $*$ \\
\hline 3rd Q & 21.2 & 25.6 & 32.5 & 28.2 & 30.5 & 20.1 & 11.9 & 17.4 & 13.8 & 10.4 & 11.9 & 9.4 & $* * *$ \\
\hline \multirow[t]{2}{*}{ 4th $\mathrm{Q}$} & 14.6 & 22.3 & 23.8 & 20.8 & 31.9 & 26.0 & 23.8 & 22.3 & 13.1 & 11.6 & 9.3 & 13.8 & $*$ \\
\hline & \multicolumn{13}{|c|}{ External Environment } \\
\hline \multicolumn{14}{|l|}{ State } \\
\hline Illinois & 24.3 & 28.3 & 27.6 & 28.8 & 28.8 & 20.3 & 16.8 & 20.3 & 15.5 & 15.7 & 17.2 & 15.9 & \\
\hline New York & 18.1 & 30.5 & 38.1 & 32.1 & 30.9 & 14.7 & 10.2 & 15.1 & 7.8 & 6.3 & 6.1 & 6.1 & $* * *$ \\
\hline \multicolumn{13}{|c|}{ HPSA- Mental Health } & \\
\hline Yes & 19.7 & 27.3 & 31.3 & 27.6 & 28.7 & 17.4 & 14.4 & 17.5 & 12.1 & 11.7 & 11.3 & 11.3 & $* * *$ \\
\hline No & 23.1 & 31.0 & 41.9 & 29.7 & 22.2 & 16.3 & 8.8 & 14.9 & 14.5 & 12.3 & 10.0 & 11.9 & $*$ \\
\hline \multicolumn{14}{|l|}{ Metro } \\
\hline Yes & 19.2 & 27.0 & 31.7 & 28.1 & 29.4 & 17.6 & 13.8 & 18.2 & 12.3 & 12 & 11.6 & 11.5 & $* * *$ \\
\hline No & 32.3 & 35.6 & 43.4 & 27.1 & 8.1 & 13.5 & 10.6 & 8.2 & 14.5 & 9.8 & 7.1 & 11.0 & $*$ \\
\hline \multicolumn{14}{|l|}{$\mathrm{CMHC}$} \\
\hline Yes & 21.2 & 26.3 & 31.3 & 27.8 & 27.1 & 17.5 & 15.4 & 19.5 & 16.6 & 15.7 & 14.9 & 14.1 & $* * *$ \\
\hline No & 19.2 & 29.3 & 34.7 & 28.2 & 28.6 & 16.9 & 11.6 & 14.9 & 8.7 & 8.2 & 7.7 & 9.0 & $* * *$ \\
\hline \multicolumn{14}{|l|}{ FQHC } \\
\hline Yes & 19.4 & 27.1 & 31.2 & 28.1 & 29.5 & 17.5 & 14.4 & 18.0 & 12.2 & 12 & 11.6 & 11.6 & $* * *$ \\
\hline No & 25.2 & 31.7 & 41.4 & 27.4 & 17.6 & 15.8 & 8.9 & 13.3 & 14.3 & 10.9 & 8.9 & 10.8 & $*$ \\
\hline
\end{tabular}


Table 2: Percentages of Depression Treatment by Types of Coexisting Chronic Physical Conditions

Among Medicaid Beneficiaries with Type 2 Diabetes Mellitus and Newly-diagnosed Depression Multi-state Medicaid Claims Database - 2000 - 2008

\begin{tabular}{|c|c|c|c|c|c|c|c|c|c|c|c|c|c|}
\hline & \multicolumn{5}{|c|}{ Antidepressants only } & \multicolumn{2}{|c|}{ Psychotherapy only } & \multicolumn{6}{|c|}{ Both Antidepressants \& Psychotherapy } \\
\hline & Dominant & $\begin{array}{c}\text { Concordant } \\
\text { Only } \\
\end{array}$ & $\begin{array}{c}\text { Discordant } \\
\text { Only }\end{array}$ & Both $^{¥}$ & Dominant & $\begin{array}{c}\text { Concordant } \\
\text { Only }\end{array}$ & $\begin{array}{c}\text { Discordant } \\
\text { Only }\end{array}$ & Both $^{¥}$ & Dominant & $\begin{array}{c}\text { Concordant } \\
\text { Only } \\
\end{array}$ & $\begin{array}{c}\text { Discordant } \\
\text { Only }\end{array}$ & Both $^{¥}$ & \\
\hline & $\%$ & $\%$ & $\%$ & $\%$ & $\%$ & $\%$ & $\%$ & $\%$ & $\%$ & $\%$ & $\%$ & $\%$ & Sig \\
\hline \multicolumn{14}{|c|}{ Median Income } \\
\hline 1st Q & 24.4 & 30.2 & 37.1 & 22.9 & 15.3 & 16.0 & 11.7 & 15.0 & 15.3 & 15.8 & 10.8 & 14.7 & $* *$ \\
\hline 2nd Q & 19.1 & 28.0 & 34.5 & 30.8 & 27.4 & 16.6 & 12.8 & 16.6 & 11.6 & 11.6 & 9.3 & 10.1 & $* * *$ \\
\hline 3rd Q & 22.7 & 29.5 & 32.4 & 32.6 & 27.9 & 17.1 & 14.5 & 16.5 & 9.6 & 9 & 12.4 & 9.7 & $* *$ \\
\hline \multicolumn{14}{|c|}{$\%$ with GT $4 \mathrm{yr}$ college education $>16 \%^{\mathrm{H}}$} \\
\hline Yes & 19.1 & 26.9 & 31.4 & 28.5 & 30.6 & 17.9 & 14.3 & 18.3 & 11.6 & 10.9 & 11.4 & 11.1 & $* * *$ \\
\hline $\begin{array}{c}\text { No } \\
\% \text { below po }\end{array}$ & $\begin{array}{r}26.7 \\
\text { level GT } 11 .\end{array}$ & $\%^{\mathrm{Ht}}$ & 43.0 & 25.8 & 10.3 & 13.6 & 8.1 & 11.8 & 18.1 & 16.3 & 8.9 & 12.9 & $* *$ \\
\hline Yes & 19.8 & 26.8 & 32.4 & 27.5 & 28.4 & 17.5 & 13.9 & 17.3 & 11.6 & 11.7 & 11.3 & 11.7 & $* * *$ \\
\hline
\end{tabular}

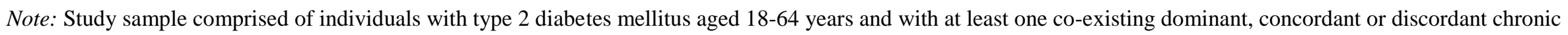

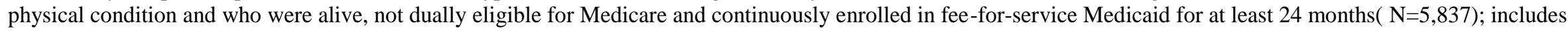
Medicaid data from three states: Illinois, Texas, New York

Asterisks $(*)$ represent significant differences in depression treatment categories i.e. antidepressants only, psychotherapy only, both antidepressants and psychotherapy and no treatment by coexisting chronic physical conditions categories i.e. Dominant, Concordant Only, Discordant Only and Both Concordant and Discordant for each of the categorical independent variable subgroups

$* * * \mathrm{P}<.001 ; * * .001 \leq \mathrm{P}<.01 ; * .01 \leq \mathrm{P}<.05$

$¥$ Both Concordant and Discordant

\#1 16\% Cut off was chosen based on 2000 Census Education attainment results

\#1 $11.1 \%$ Cut off was chosen based on 1999 Census Poverty levels

Q: Quartile; HPSA: Health Professional Shortage Area; CMHC: Community Mental Health Clinic; FQHC: Federally qualified health clinic; GT: Greater Than; 
Table 3: Odds Ratios and Adjusted Odds Ratio and 95\% Confidence Intervals of Types of Co-existing Chronic Physical Conditions

from Multinomial Logistic Regression on Depression Treatment Categories Among Medicaid Beneficiaries with Type 2 Diabetes Mellitus and Newly-diagnosed Depression Multi-state Medicaid Claims Database - 2000 - 2008 Antidepressants Only Psychotherapy Only

Both Antidepressants \& Psychotherapy

\begin{tabular}{|c|c|c|c|c|c|c|c|c|c|}
\hline & OR & $95 \% \mathrm{CI}$ & Sig & OR & $95 \% \mathrm{CI}$ & Sig & OR & $95 \% \mathrm{CI}$ & Sig \\
\hline & \multicolumn{9}{|c|}{ Model 1: Adjusted for Types of Co-existing Chronic Physical Conditions } \\
\hline Dominant & 0.65 & $0.51,0.83$ & $* * *$ & 2.23 & $1.72,2.91$ & $* * *$ & 1.21 & $0.89,1.65$ & \\
\hline Concordant Only & 0.83 & $0.68,1.00$ & & 1.26 & $0.98,1.62$ & & 1.05 & $0.80,1.38$ & \\
\hline Discordant Only & & Reference & & & Reference & & & Reference & \\
\hline \multirow[t]{2}{*}{ Both $^{t}$} & 0.82 & $0.69,0.98$ & $*$ & 1.24 & $0.98,1.57$ & & 1.01 & $0.78,1.30$ & \\
\hline & AOR & $95 \%$ CI & Sig & AOR & $95 \%$ CI & Sig & AOR & $95 \%$ CI & Sig \\
\hline
\end{tabular}

Model 2: Adjusted for Model I + Predisposing (gender, age, race/ethnicity), Need Factors (other mental health conditions, number of conditions), Enabling Factors (Medicaid Eligibility Categories-Poverty, Medical Need, Waiver) Personal health practices (number of ER and outpatient visits, inpatient hospitalization, number of OAD classes, insulin use and polypharmacy)

\begin{tabular}{|c|c|c|c|c|c|c|c|c|}
\hline Dominant & 0.77 & $0.60,1.00$ & $*$ & 1.61 & $1.21,2.15$ & $* *$ & 1.14 & $0.82,1.60$ \\
\hline Concordant Only & 0.81 & $0.67,1.00$ & * & 1.31 & $1.01,1.70$ & $*$ & 1.03 & $0.78,1.38$ \\
\hline Discordant Only & & Reference & & & Reference & & & Reference \\
\hline Both $^{\dagger}$ & 0.88 & $0.73,1.07$ & & 1.12 & $0.87,1.44$ & & 0.87 & $0.66,1.14$ \\
\hline
\end{tabular}

Model 3: Adjusted for Model 1+Model 2+ External environment characteristics (whether county of residence had a CMHC, FQHC, was HPSA for mental health, density of social workers rural/urban status of county, median income in the county, whether percent below poverty level and percent with college education greater than national average)
Dominant
Discordant Only
Both $^{\mathrm{t}}$

$0.77 \quad 0.59,0.99$

1.60

$1.20,2.13$

$* *$

$1.11 \quad 0.79,1.55$

$0.80 \quad 0.65,0.98$

$1.321 .01,1.72$

$1.010 .76,1.35$ Reference

Reference

Reference

$0.87 \quad 0.72,1.05$

$1.140 .88,1.46$

$0.86 \quad 0.65,1.13$

Note: Study sample comprised of individuals with type 2 diabetes mellitus aged 18-64 years and with at least one coexisting concordant, discordant or dominant chronic physical condition and who were alive, not dually eligible for Medicare and continuously enrolled in fee-for-service Medicaid for at least 24 months( $\mathrm{N}=5,837)$; includes Medicaid data from three states: Illinois, Texas, New York

Asterisks $(*)$ indicate statistical significance are based on multinomial logistic regression with discordant conditions as reference group for independent variable and no antidepressant treatment i.e. none as reference group for dependent variable.

$* * * \mathrm{P}<0.001 ; * * 0.001 \leq \mathrm{P}<0.01 ; * 0.01 \leq \mathrm{P}<0.05$.

¥ Both Concordant and Discordant

ER: Emergency Room; OAD: Oral Antidiabetic Drugs; HPSA: Health Professional Shortage Area; CMHC:

Community Mental Health Clinic; FQHC: Federally qualified health clinic 
Appendix A : ICD-9-CM Codes for Identifying Types of Coexisting chronic physical Conditions

\begin{tabular}{|c|c|}
\hline Conditions & ICD-9-CM Codes \\
\hline $\begin{array}{l}\text { Concordant conditions } \\
\text { Coronary Artery Disease }\end{array}$ & $\begin{array}{l}410,4100,4101,4102,4103,4104,4105,4106,4107,4108,4109,411,4110, \\
4111,4118,41181,41189,412,413,4130,4131,4139,414,4140,41400,414 \\
01,41402,41403,41404,41405,4141,41410,41411,41419,4148,4149\end{array}$ \\
\hline Congestive Heart Failure & $40201,40211,40291,40401,40411,40491,428,4280,4281,4289$ \\
\hline Arrhythmia & $423,4230,4231,4232,4238,4239,42731$ \\
\hline Stroke & $\begin{array}{l}\text { 431,43301,43311,43321,43331,43381,43391,43401,43411,43491,435, } \\
\text { 4350,4351,4352,4353,4358,4359,438,4380,4381,43811,43812,4382,43 } \\
\text { 83,4384,4385,43850,4385,43852,43853,4388,43881, } \\
\text { 43882,43889,4389 }\end{array}$ \\
\hline Peripheral Vascular Disease & $\begin{array}{l}2507,4402,44020,44021,44022,44023,44024,44029,4408,4409,4422,4 \\
423,443,4430,4431,4438,44381,44389,4439,44422,44481\end{array}$ \\
\hline Peripheral Vascular Disease-gangrene & 7854 \\
\hline $\begin{array}{l}\text { Renal } \\
\text { Chronic Renal Failure } \\
\text { Chronic Pathophysiology }\end{array}$ & $\begin{array}{l}40311,40391,40412,40413,40492,40493,585,586,587, \\
2741,27410,27411,27419, \\
40310,40390,40410,40411,40490,40491, \\
581,5810,5811,5812,5813,5818,5819, \\
582,5820,5821,5822,5824,5828,58281,58289,5829,583,5830,5831,583 \\
2,5834,5836, \\
5837,5838,58381,58389,5839,5900,59000,59001,5936,5939, \\
75312,75313,75314\end{array}$ \\
\hline Diabetic Nephropathy & $2504,25040,25041,25042,25043$ \\
\hline Acute Renal Failure and Disease & $\begin{array}{l}40300,40301,40400,40401,40402,40403,40501,4533,584,5845,5846,5 \\
847,5848,5849, \\
580,5800,5804,5808,58081,58089,5809,5901,59010,59011,5902,5903, \\
5908,59080, \\
59081,59381,866,8660,86600,86601,86602,86603,8661,86610,86611, \\
86612,86613\end{array}$ \\
\hline $\begin{array}{l}\text { Retinopathy } \\
\text { (excludes advanced retinopathy, blindness) }\end{array}$ & $3620,36201,25050,25051,25052,25053$ \\
\hline Ulcer & $700,68110,68111,6827,7071,73076,73077$ \\
\hline $\begin{array}{l}\text { Other Diabetes Related Complications } \\
\text { Uncontrolled Diabetes } \\
\text { Short Term Diabetes } \\
\text { Discordant Conditions }\end{array}$ & $\begin{array}{l}25002,25003,25010,25011,25012,25013,25020,25021,25022,25023,25 \\
030,25031, \\
25032,25033\end{array}$ \\
\hline $\begin{array}{l}\text { Gastro Intestinal Tract Related Disorders: } \\
\text { GERD/Esophagitis } \\
\text { Peptic ulcers } \\
\text { Inflammatory Bowel Disease } \\
\text { Diverticulitis } \\
\text { Gall Bladder disease and stone } \\
\text { Viral hepatitis }\end{array}$ & $\begin{array}{l}5301,5302,5303,53081,531,532,533,534,555,556,56211,56213,574 \\
575,576,070\end{array}$ \\
\hline Chronic Obstructive Pulmonary Disease & $490,491,492,493,495,496,500,501,502,503,504,505,5064$ \\
\hline Gout & 274,712 \\
\hline Hip problem & $\begin{array}{l}\text { 71905,71915,71925,71935,71945,71955,71965,71975,71985,71995,72 } \\
65,73314,73315,73342,820 \\
720,7213,72142,72210,72252,72273,72283, \\
72293,72402,7242,7243,7244,7245,7246,7247,7248,7249\end{array}$ \\
\hline
\end{tabular}

\section{Page 98 of 173}


Appendix A : ICD-9-CM Codes for Identifying Types of Coexisting chronic physical Conditions

\begin{tabular}{|c|c|}
\hline Conditions & ICD-9-CM Codes \\
\hline Osteoarthritis & 715 \\
\hline Other Arthritis & 716 \\
\hline Rheumatoid arthritis & 714 \\
\hline Connective tissue rheumatological disease & $7100,7101,7104,725$ \\
\hline Blindness Single Eye & $3696,3697,3698,3699$ \\
\hline \multicolumn{2}{|l|}{ Dominant Conditions } \\
\hline End Stage Renal Disease & E8791,V51,V56,V560,V5631,V5632,V568 \\
\hline End Stage Liver Disease & $5722,5723,5724,5728,4560,4561,4562,45620,45621,571$ \\
\hline Blindness Both Eyes/ Advanced Retinopathy & $36202,3690,3691,3692,3693,3694$ \\
\hline Cancer & $\begin{array}{l}140,141,142,143,144,145,146,147,148,149,150,151,152,153,154,155,1 \\
56,157,158,159,160,161,162,163,164,165,166,167,168,169,170,171,17 \\
2,174,175,176,177,178,179,180,181,182,183,184,186,187,188,189,190 \\
, 191,192,193,194,195,196,197,198,199,200,201,202,203,204,205,206, \\
207,208\end{array}$ \\
\hline Pre-dementia Cognitive Impairment & $294,2941,29283,2949,33183,78093,438,3330,3334,3315$ \\
\hline Dementia and Related Conditions & $\begin{array}{l}2900,29010,29011,29012,29013,2902,29021,2903,29040,29041,29042 \\
\text {,29043,2912, } \\
29410,29411,2948,3310,3311,3312,3317,33182,33189,3319,3320,046 \\
1,0463,0941, \\
29282,3109\end{array}$ \\
\hline Multiple Sclerosis & 340 \\
\hline Hemiplegia Hemiparesis and Paraplegia & 342,3441 \\
\hline Parkinson's Disease & 332 \\
\hline Muscular dystrophy & 359 \\
\hline Spinal cord injury & $\begin{array}{l}80600,80601,80602,80603,80604,80605,80606,80607,80608,80609,80 \\
61,9520, \\
34400,34401,34402,34403,34404,34409\end{array}$ \\
\hline Epilepsy & 345 \\
\hline Gastropareis & 5363 \\
\hline AIDS & 042 \\
\hline
\end{tabular}


Appendix B: Odds Ratios and Adjusted Odds Ratio and 95\% Confidence Intervals of All Independent Variables from Multinomial Logistic Regression on Depression Treatment Categories Results presented for Antidepressants Only

Among Medicaid Beneficiaries with Type 2 Diabetes Mellitus and Newly-diagnosed Depression Multi-state Medicaid Claims Database - 2000 - 2008

\begin{tabular}{|c|c|c|c|c|c|c|c|c|c|}
\hline \multirow[b]{2}{*}{ Characteristics } & \multicolumn{9}{|c|}{ Antidepressants Only } \\
\hline & OR & $95 \% \mathrm{CI}$ & Sig & AOR & $95 \% \mathrm{CI}$ & Sig & AOR & $95 \% \mathrm{CI}$ & Sig \\
\hline \multicolumn{10}{|c|}{ Types of Coexisting Chronic Physical Conditions } \\
\hline Dominant & 0.65 & {$[0.51,0.83]$} & $* * *$ & 0.77 & {$[0.60,1.00]$} & $*$ & 0.77 & {$[0.59,0.99]$} & $*$ \\
\hline Concordant Only & 0.83 & {$[0.68,1.00]$} & & 0.81 & {$[0.67,1.00]$} & $*$ & 0.80 & {$[0.65,0.98]$} & $*$ \\
\hline Discordant Only & & & & & & & & & \\
\hline Both & 0.82 & {$[0.69,0.98]$} & $*$ & 0.88 & {$[0.73,1.07]$} & & 0.87 & {$[0.72,1.05]$} & \\
\hline \multicolumn{10}{|l|}{ Age } \\
\hline $18-44$ years & & & & & & & & & \\
\hline $45-54$ years & & & & 1.04 & {$[0.87,1.23]$} & & 1.05 & {$[0.88,1.25]$} & \\
\hline $55-64$ years & & & & 1.03 & {$[0.87,1.22]$} & & 1.06 & {$[0.89,1.26]$} & \\
\hline \multicolumn{10}{|l|}{ Female } \\
\hline Female & & & & 1.17 & {$[1.01,1.34]$} & $*$ & 1.14 & {$[0.99,1.32]$} & \\
\hline Male & & & & & & & & & \\
\hline \multicolumn{10}{|l|}{ Race } \\
\hline White & & & & 1.63 & {$[1.37,1.95]$} & $* * *$ & 1.47 & {$[1.21,1.78]$} & $* * *$ \\
\hline African American & & & & & & & & & \\
\hline Others & & & & 1.33 & {$[1.13,1.57]$} & $* * *$ & 1.30 & {$[1.10,1.53]$} & $* *$ \\
\hline \multicolumn{10}{|l|}{ Other Mental Health Conditions } \\
\hline Yes & & & & 0.63 & {$[0.54,0.73]$} & $* * *$ & 0.65 & {$[0.55,0.75]$} & $* * *$ \\
\hline No & & & & & & & & & \\
\hline \multicolumn{10}{|l|}{ Medicaid Eligibility-Poverty } \\
\hline Yes & & & & 0.63 & {$[0.47,0.83]$} & $* *$ & 0.61 & {$[0.46,0.81]$} & $* * *$ \\
\hline No & & & & & & & & & \\
\hline \multicolumn{10}{|c|}{ Medicaid Eligibility-Medical Needs } \\
\hline Yes & & & & 0.89 & {$[0.68,1.17]$} & & 0.87 & {$[0.66,1.14]$} & \\
\hline No & & & & & & & & & \\
\hline \multicolumn{10}{|l|}{ Medicaid Eligibility-Waiver } \\
\hline Yes & & & & 1.21 & {$[0.87,1.70]$} & & 1.14 & {$[0.81,1.60]$} & \\
\hline No & & & & & & & & & \\
\hline \multicolumn{10}{|l|}{ Oral Antidiabetic Drugs (OADs) } \\
\hline $1 \mathrm{OAD}$ & & & & 0.83 & {$[0.65,1.06]$} & & 0.82 & {$[0.64,1.05]$} & \\
\hline $2 \mathrm{OAD}$ & & & & 0.89 & {$[0.70,1.15]$} & & 0.89 & {$[0.69,1.14]$} & \\
\hline $3 \mathrm{OAD}$ & & & & & & & & & \\
\hline No OAD & & & & 0.73 & {$[0.57,0.94]$} & $*$ & 0.73 & {$[0.57,0.94]$} & $*$ \\
\hline
\end{tabular}


Appendix B: Odds Ratios and Adjusted Odds Ratio and 95\% Confidence Intervals

of All Independent Variables from Multinomial Logistic Regression on Depression Treatment Categories

Results presented for Antidepressants Only

Among Medicaid Beneficiaries with Type 2 Diabetes Mellitus and Newly-diagnosed Depression

Multi-state Medicaid Claims Database - 2000 - 2008

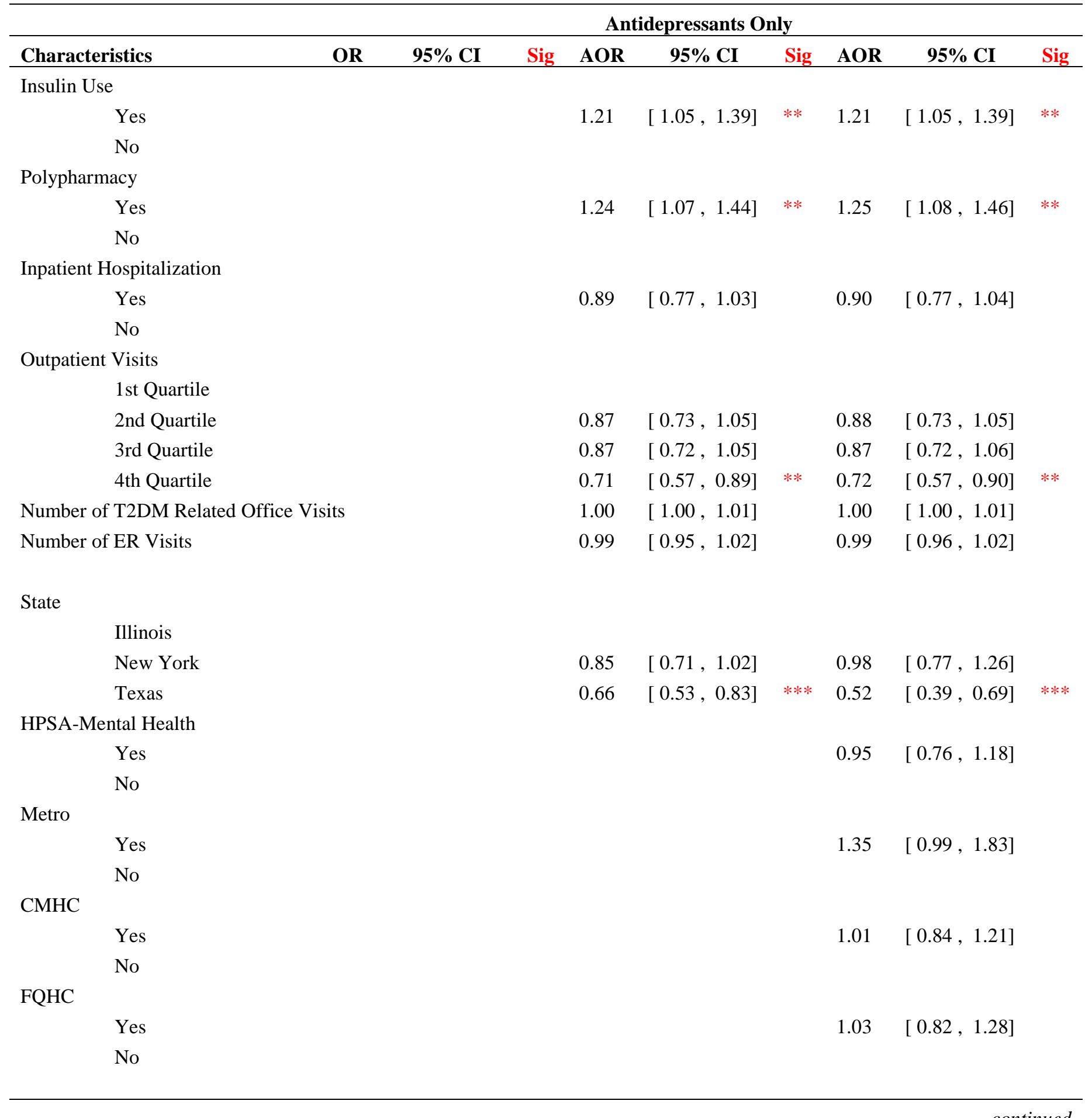


Appendix B: Odds Ratios and Adjusted Odds Ratio and 95\% Confidence Intervals

of All Independent Variables from Multinomial Logistic Regression on Depression Treatment Categories

Results presented for Antidepressants Only

Among Medicaid Beneficiaries with Type 2 Diabetes Mellitus and Newly-diagnosed Depression

Multi-state Medicaid Claims Database - 2000 - 2008

\begin{tabular}{|c|c|c|c|c|c|c|c|c|c|}
\hline \multirow[b]{2}{*}{ Characteristics } & \multicolumn{9}{|c|}{ Antidepressants Only } \\
\hline & OR & $95 \% \mathrm{CI}$ & Sig & AOR & $95 \% \mathrm{CI}$ & Sig & AOR & $95 \% \mathrm{CI}$ & Sig \\
\hline Density Social Workers & & & & & & & 0.90 & {$[0.83,0.99]$} & $*$ \\
\hline \multicolumn{10}{|l|}{ Median Household Income } \\
\hline 1 st Quartile & & & & & & & 1.18 & {$[0.81,1.71]$} & \\
\hline 2nd Quartile & & & & & & & 1.07 & {$[0.82,1.41]$} & \\
\hline 3rd Quartile & & & & & & & 1.17 & {$[0.89,1.53]$} & \\
\hline \multicolumn{10}{|l|}{ 4th Quartile } \\
\hline \multicolumn{10}{|c|}{$\%$ with GT 4 yr college education $>16 \%$} \\
\hline \multicolumn{10}{|c|}{ Yes } \\
\hline No & & & & & & & 0.79 & {$[0.61,1.03]$} & \\
\hline \multicolumn{10}{|c|}{$\%$ below poverty level GT $11.1 \%{ }^{\mathrm{H}}$} \\
\hline Yes & & & & & & & 0.94 & {$[0.70,1.27]$} & \\
\hline No & & & & & & & 0.92 & {$[0.71,1.19]$} & \\
\hline
\end{tabular}

Asterisks $(*)$ indicate statistical significance are based on multinomial logistic regression with discordant conditions as reference group for independent variable and no antidepressant treatment i.e. none as reference group for dependent variable.

$* * * \mathrm{P}<0.001 ; * * 0.001 \leq \mathrm{P}<0.01 ; * 0.01 \leq \mathrm{P}<0.05$.

\#16\% Cut off was chosen based on 2000 Census Education attainment results

H1 $11.1 \%$ Cut off was chosen based on 1999 Census Poverty levels

HPSA: Health Professional Shortage Area; CMHC: Community Mental Health Clinic; FQHC: Federally qualified health clinic; 
Appendix C: Odds Ratios and Adjusted Odds Ratio and 95\% Confidence Intervals of All Independent Variables from Multinomial Logistic Regression on Depression Treatment Categories Results presented for Psychotherapy Only

Among Medicaid Beneficiaries with Type 2 Diabetes Mellitus and Newly-diagnosed Depression Multi-state Medicaid Claims Database - 2000 - 2008

\begin{tabular}{|c|c|c|c|c|c|c|c|c|c|}
\hline \multirow[b]{2}{*}{ Characteristics } & \multicolumn{9}{|c|}{ Psychotherapy Only } \\
\hline & OR & $95 \% \mathrm{CI}$ & Sig & AOR & $95 \% \mathrm{CI}$ & Sig & AOR & $95 \% \mathrm{CI}$ & Sig \\
\hline \multicolumn{10}{|c|}{ Types of Coexisting Chronic Physical Conditions } \\
\hline Dominant & 2.23 & {$[1.72,2.91]$} & $* * *$ & 1.61 & {$[1.21,2.15]$} & $* *$ & 1.60 & {$[1.20,2.13]$} & $* *$ \\
\hline Concordant Only & 1.26 & {$[0.98,1.62]$} & & 1.31 & {$[1.01,1.70]$} & $*$ & 1.32 & {$[1.01,1.72]$} & $*$ \\
\hline Discordant Only & & & & & & & & & \\
\hline Both & 1.24 & {$[0.98,1.57]$} & & 1.12 & {$[0.87,1.44]$} & & 1.14 & {$[0.88,1.46]$} & \\
\hline \multicolumn{10}{|l|}{ Age } \\
\hline \multicolumn{10}{|l|}{$18-44$ years } \\
\hline 45-54 years & & & & 1.09 & {$[0.89,1.33]$} & & 1.06 & {$[0.86,1.30]$} & \\
\hline $55-64$ years & & & & 1.25 & {$[1.02,1.52]$} & $*$ & 1.20 & {$[0.98,1.47]$} & \\
\hline \multicolumn{10}{|l|}{ Female } \\
\hline Female & & & & 0.63 & {$[0.54,0.74]$} & $* * *$ & 0.65 & {$[0.56,0.76]$} & $* * *$ \\
\hline Male & & & & & & & & & \\
\hline \multicolumn{10}{|l|}{ Race } \\
\hline White & & & & 0.90 & {$[0.74,1.10]$} & & 1.13 & {$[0.91,1.39]$} & \\
\hline African American & & & & & & & & & \\
\hline Others & & & & 0.86 & {$[0.71,1.04]$} & & 0.90 & {$[0.74,1.09]$} & \\
\hline \multicolumn{10}{|l|}{ Other Mental Health Conditions } \\
\hline Yes & & & & 1.99 & {$[1.70,2.34]$} & $* * *$ & 1.93 & {$[1.64,2.28]$} & $* * *$ \\
\hline No & & & & & & & & & \\
\hline \multicolumn{10}{|l|}{ Medicaid Eligibility-Poverty } \\
\hline Yes & & & & 1.16 & {$[0.85,1.59]$} & & 1.17 & {$[0.85,1.60]$} & \\
\hline No & & & & & & & & & \\
\hline \multicolumn{10}{|c|}{ Medicaid Eligibility-Medical Needs } \\
\hline Yes & & & & 1.64 & {$[1.25,2.15]$} & $* * *$ & 1.64 & {$[1.24,2.17]$} & $* * *$ \\
\hline No & & & & & & & & & \\
\hline \multicolumn{10}{|l|}{ Medicaid Eligibility-Waiver } \\
\hline Yes & & & & 0.67 & {$[0.43,1.05]$} & & 0.69 & {$[0.44,1.07]$} & \\
\hline No & & & & & & & & & \\
\hline \multicolumn{10}{|c|}{ Oral Antidiabetic Drugs (OADs) } \\
\hline $1 \mathrm{OAD}$ & & & & 0.85 & {$[0.62,1.17]$} & & 0.87 & {$[0.63,1.20]$} & \\
\hline $2 \mathrm{OAD}$ & & & & 0.81 & {$[0.59,1.13]$} & & 0.82 & {$[0.59,1.14]$} & \\
\hline $3 \mathrm{OAD}$ & & & & & & & & & \\
\hline No OAD & & & & 1.32 & {$[0.97,1.80]$} & & 1.34 & {$[0.98,1.83]$} & \\
\hline
\end{tabular}


Appendix C: Odds Ratios and Adjusted Odds Ratio and 95\% Confidence Intervals of All Independent Variables from Multinomial Logistic Regression on Depression Treatment Categories Results presented for Psychotherapy Only

Among Medicaid Beneficiaries with Type 2 Diabetes Mellitus and Newly-diagnosed Depression Multi-state Medicaid Claims Database - 2000 - 2008

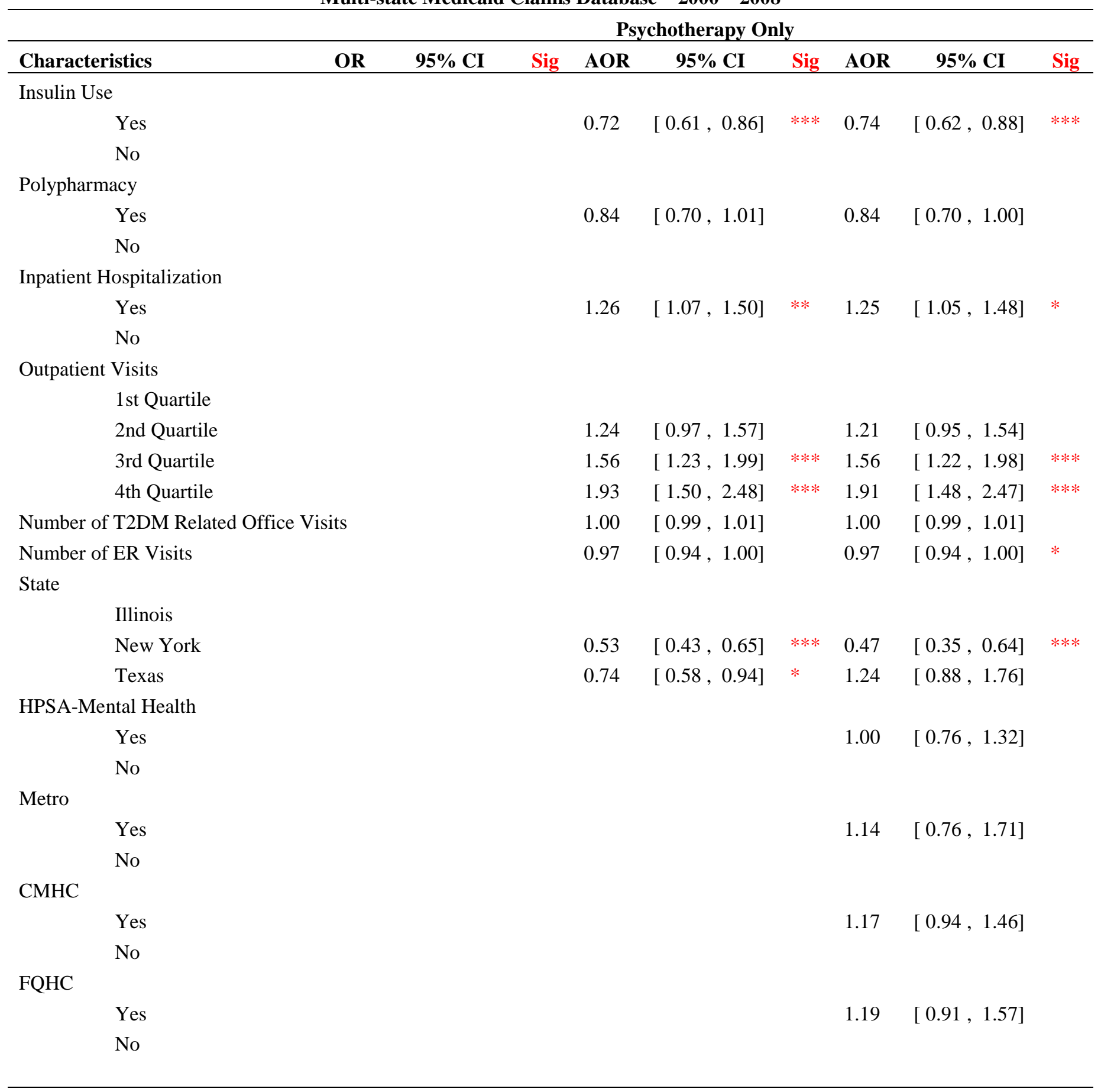


Appendix C: Odds Ratios and Adjusted Odds Ratio and 95\% Confidence Intervals of All Independent Variables from Multinomial Logistic Regression on Depression Treatment Categories Results presented for Psychotherapy Only

Among Medicaid Beneficiaries with Type 2 Diabetes Mellitus and Newly-diagnosed Depression Multi-state Medicaid Claims Database - 2000 - 2008

\begin{tabular}{|c|c|c|c|c|c|c|c|c|c|}
\hline \multirow[b]{2}{*}{ Characteristics } & \multicolumn{9}{|c|}{ Psychotherapy Only } \\
\hline & OR & $95 \% \mathrm{CI}$ & Sig & AOR & $95 \% \mathrm{CI}$ & Sig & AOR & $95 \% \mathrm{CI}$ & Sig \\
\hline Density Social Workers & & & & & & & 1.14 & {$[1.01,1.28]$} & $*$ \\
\hline \multicolumn{10}{|l|}{ Median Household Income } \\
\hline 1st Quartile & & & & & & & 1.14 & {$[0.75,1.74]$} & \\
\hline 2nd Quartile & & & & & & & 1.12 & {$[0.81,1.53]$} & \\
\hline 3rd Quartile & & & & & & & 1.18 & {$[0.86,1.61]$} & \\
\hline \multicolumn{10}{|l|}{ 4th Quartile } \\
\hline \multicolumn{10}{|c|}{$\%$ with GT $4 y r$ college education $>16 \% 1$} \\
\hline \multicolumn{10}{|l|}{ Yes } \\
\hline No & & & & & & & 1.80 & {$[1.30,2.51]$} & $* * *$ \\
\hline \multicolumn{10}{|c|}{$\%$ below poverty level GT $11.1 \%^{H}$} \\
\hline Yes & & & & & & & 0.98 & {$[0.68,1.42]$} & \\
\hline No & & & & & & & 1.38 & {$[0.97,1.96]$} & \\
\hline
\end{tabular}

Asterisks (*) indicate statistical significance are based on multinomial logistic regression with discordant conditions as reference group for independent variable and no antidepressant treatment i.e. none as reference group for dependent variable.

$* * * \mathrm{P}<0.001 ; * * 0.001 \leq \mathrm{P}<0.01 ; * 0.01 \leq \mathrm{P}<0.05$.

\#16\% Cut off was chosen based on 2000 Census Education attainment results

Hł $11.1 \%$ Cut off was chosen based on 1999 Census Poverty levels

HPSA: Health Professional Shortage Area; CMHC: Community Mental Health Clinic; FQHC: Federally qualified health clinic; 
Appendix D: Odds Ratios and Adjusted Odds Ratio and 95\% Confidence Intervals of All Independent Variables from Multinomial Logistic Regression on Depression Treatment Categories Results presented for Both Antidepressants and Psychotherapy Among Medicaid Beneficiaries with Type 2 Diabetes Mellitus and Newly-diagnosed Depression Multi-state Medicaid Claims Database - 2000 - 2008

Both Antidepressants \&Psychotherapy

\begin{tabular}{|c|c|c|c|c|c|c|c|c|c|}
\hline \multirow{2}{*}{ Characteristics } & \multicolumn{9}{|c|}{ Both Antidepressants \&Psychotherapy } \\
\hline & OR & $95 \% \mathrm{CI}$ & Sig & AOR & $95 \% \mathrm{CI}$ & Sig & AOR & $95 \% \mathrm{CI}$ & Sig \\
\hline \multicolumn{10}{|c|}{ Types of Coexisting Chronic Physical Conditions } \\
\hline Dominant & 1.21 & {$[0.89,1.65]$} & & 1.14 & {$[0.82,1.60]$} & & 1.11 & {$[0.79,1.55]$} & \\
\hline Concordant Only & 1.05 & {$[0.80,1.38]$} & & 1.03 & {$[0.78,1.38]$} & & 1.01 & {$[0.76,1.35]$} & \\
\hline Discordant Only & & & & & & & & & \\
\hline Both & 1.01 & {$[0.78,1.30]$} & & 0.87 & {$[0.66,1.14]$} & & 0.86 & {$[0.65,1.13]$} & \\
\hline \multicolumn{10}{|l|}{ Age } \\
\hline \multicolumn{10}{|l|}{$18-44$ years } \\
\hline $45-54$ years & & & & 1.07 & {$[0.86,1.34]$} & & 1.04 & {$[0.83,1.31]$} & \\
\hline $55-64$ years & & & & 0.85 & {$[0.67,1.07]$} & & 0.82 & {$[0.65,1.04]$} & \\
\hline \multicolumn{10}{|l|}{ Female } \\
\hline Female & & & & 1.02 & {$[0.84,1.24]$} & & 1.05 & {$[0.86,1.27]$} & \\
\hline Male & & & & & & & & & \\
\hline \multicolumn{10}{|l|}{ Race } \\
\hline White & & & & 1.45 & {$[1.15,1.83]$} & ** & 1.85 & {$[1.44,2.38]$} & **** \\
\hline African American & & & & & & & & & \\
\hline Others & & & & 1.21 & {$[0.96,1.52]$} & & 1.21 & {$[0.96,1.53]$} & \\
\hline \multicolumn{10}{|c|}{ Other Mental Health Conditions } \\
\hline Yes & & & & 0.96 & {$[0.79,1.17]$} & & 0.95 & {$[0.78,1.16]$} & \\
\hline \multicolumn{10}{|l|}{ No } \\
\hline \multicolumn{10}{|l|}{ Medicaid Eligibility-Poverty } \\
\hline Yes & & & & 1.00 & {$[0.71,1.42]$} & & 0.98 & {$[0.69,1.39]$} & \\
\hline \multicolumn{10}{|l|}{ No } \\
\hline \multicolumn{10}{|c|}{ Medicaid Eligibility-Medical Needs } \\
\hline Yes & & & & 1.61 & {$[1.18,2.21]$} & ** & 1.63 & {$[1.18,2.24]$} & ** \\
\hline \multicolumn{10}{|l|}{ No } \\
\hline \multicolumn{10}{|l|}{ Medicaid Eligibility-Waiver } \\
\hline Yes & & & & 1.32 & {$[0.79,2.21]$} & & 1.23 & {$[0.73,2.07]$} & \\
\hline \multicolumn{10}{|l|}{ No } \\
\hline \multicolumn{10}{|c|}{ Oral Antidiabetic Drugs (OADs) } \\
\hline $1 \mathrm{OAD}$ & & & & 0.67 & {$[0.48,0.93]$} & * & 0.66 & {$[0.47,0.92]$} & * \\
\hline $2 \mathrm{OAD}$ & & & & 0.65 & {$[0.46,0.91]$} & $*$ & 0.64 & {$[0.45,0.90]$} & $* *$ \\
\hline \multicolumn{10}{|l|}{3 OAD } \\
\hline No OAD & & & & 0.61 & {$[0.44,0.85]$} & ** & 0.61 & {$[0.44,0.86]$} & ** \\
\hline \multicolumn{10}{|l|}{ Insulin Use } \\
\hline Yes & & & & 1.22 & {$[1.01,1.48]$} & $*$ & 1.26 & {$[1.04,1.52]$} & $*$ \\
\hline \multicolumn{10}{|l|}{ No } \\
\hline \multicolumn{10}{|l|}{ Polypharmacy } \\
\hline Yes & & & & 1.34 & {$[1.09,1.64]$} & ** & 1.35 & {$[1.10,1.66]$} & ** \\
\hline No & & & & & & & & & \\
\hline \multicolumn{10}{|l|}{ Inpatient Hospitalization } \\
\hline Yes & & & & 1.18 & {$[0.97,1.44]$} & & 1.16 & {$[0.95,1.42]$} & \\
\hline No & & & & & & & & & \\
\hline
\end{tabular}


Appendix D: Odds Ratios and Adjusted Odds Ratio and 95\% Confidence Intervals

of All Independent Variables from Multinomial Logistic Regression on Depression Treatment Categories Results presented for Both Antidepressants and Psychotherapy

Among Medicaid Beneficiaries with Type 2 Diabetes Mellitus and Newly-diagnosed Depression Multi-state Medicaid Claims Database - 2000 - 2008

\begin{tabular}{|c|c|c|c|c|c|c|c|c|}
\hline \multirow{2}{*}{ Characteristics } & \multicolumn{8}{|c|}{ Both Antidepressants \&Psychotherapy } \\
\hline & 95\% CI & Sig & AOR & $95 \% \mathrm{CI}$ & Sig & AOR & $95 \% \mathrm{CI}$ & Sig \\
\hline \multicolumn{9}{|l|}{ Outpatient Visits } \\
\hline \multicolumn{9}{|l|}{ 1st Quartile } \\
\hline 2nd Quartile & & & 0.96 & {$[0.74,1.24]$} & & 0.96 & {$[0.74,1.24]$} & \\
\hline 3rd Quartile & & & 0.94 & {$[0.72,1.23]$} & & 0.93 & {$[0.71,1.23]$} & \\
\hline 4th Quartile & & & 1.19 & {$[0.89,1.59]$} & & 1.17 & {$[0.87,1.57]$} & \\
\hline Number of T2DM Related Office Visits & & & 1.00 & {$[0.99,1.01]$} & & 1.00 & {$[0.99,1.01]$} & \\
\hline Number of ER Visits & & & 1.00 & {$[0.97,1.03]$} & & 1.00 & {$[0.97,1.04]$} & \\
\hline \multicolumn{9}{|l|}{ State } \\
\hline Illinois & & & & & & & & \\
\hline New York & & & 0.32 & {$[0.25,0.42]$} & $* * *$ & 0.35 & {$[0.25,0.50]$} & $* * *$ \\
\hline Texas & & & 1.05 & {$[0.81,1.37]$} & & 1.56 & {$[1.08,2.25]$} & * \\
\hline \multicolumn{9}{|l|}{ HPSA-Mental Health } \\
\hline Yes & & & & & & 0.99 & {$[0.74,1.33]$} & \\
\hline No & & & & & & & & \\
\hline \multicolumn{9}{|l|}{ Metro } \\
\hline Yes & & & & & & 1.25 & {$[0.82,1.90]$} & \\
\hline No & & & & & & & & \\
\hline \multicolumn{9}{|l|}{ CMHC } \\
\hline Yes & & & & & & 1.42 & {$[1.12,1.81]$} & $* *$ \\
\hline No & & & & & & & & \\
\hline \multicolumn{9}{|l|}{ FQHC } \\
\hline Yes & & & & & & 1.38 & {$[1.02,1.85]$} & * \\
\hline No & & & & & & & & \\
\hline Density Social Workers & & & & & & 1.05 & {$[0.93,1.19]$} & \\
\hline \multicolumn{9}{|l|}{ Median Household Income } \\
\hline 1st Quartile & & & & & & 0.91 & {$[0.57,1.44]$} & \\
\hline 2nd Quartile & & & & & & 0.89 & {$[0.62,1.27]$} & \\
\hline 3rd Quartile & & & & & & 0.94 & {$[0.66,1.35]$} & \\
\hline 4th Quartile & & & & & & & & \\
\hline \multirow{2}{*}{\multicolumn{9}{|c|}{$\begin{array}{c}\% \text { with GT } 4 \text { yr college education }>16 \% 1 \\
\text { Yes }\end{array}$}} \\
\hline & & & & & & & & \\
\hline No & & & & & & 1.17 & {$[0.84,1.63]$} & \\
\hline \multicolumn{9}{|l|}{$\%$ below poverty level GT $11.1 \%^{\mathrm{H}}$} \\
\hline Yes & & & & & & 1.04 & {$[0.69,1.57]$} & \\
\hline No & & & & & & 1.02 & {$[0.71,1.47]$} & \\
\hline
\end{tabular}

Asterisks $(*)$ indicate statistical significance are based on multinomial logistic regression with discordant conditions as reference group for independent variable and no antidepressant treatment i.e. none as reference group for dependent variable.

$* * * \mathrm{P}<0.001 ; * * 0.001 \leq \mathrm{P}<0.01 ; * 0.01 \leq \mathrm{P}<0.05$.

\# 16\% Cut off was chosen based on 2000 Census Education attainment results

H111.1\% Cut off was chosen based on 1999 Census Poverty levels

HPSA: Health Professional Shortage Area; CMHC: Community Mental Health Clinic; FQHC: Federally qualified health clinic; 
CHAPTER 4

Page $\mathbf{1 0 8}$ of $\mathbf{1 7 3}$ 


\section{CHAPTER 4: Treatment for Depression and Healthcare Expenditures among Medicaid Beneficiaries with Type 2 Diabetes Mellitus and Newly-Diagnosed Depression}

\section{Introduction}

\section{Type 2 Diabetes Mellitus (T2DM), Depression and Healthcare Expenditures}

Individuals with coexisting type 2 diabetes mellitus (T2DM) and depression use more healthcare services such as inpatient [1], outpatient [2,3] and prescription drug use [2,3], report higher total $[1,3,4]$ as well as T2DM-related medical care expenditures, as compared individuals with T2DM and no depression [5]. Research with Medical Expenditure Panel Survey, a nationally representative data of the United States' population has shown that among individuals with diabetes, those with coexisting depression could have mean total annual healthcare expenditures 4.5 times higher $(\$ 247,000,000)$ compared to those without depression $(\$ 55,000,000)$ [3]. An analysis of West Virginia state Medicaid data (1997-2002) showed that among enrollees with T2DM, those with depression had 65\% higher healthcare expenditures as compared to those without depression [6]. Therefore, presence of depression is associated with substantially high healthcare expenditures among individuals with T2DM. However, in general, recovery from depression following depression treatment has been shown to be associated with lower subsequent healthcare utilization and expenditures [7].

\section{Treatment for Depression and Healthcare Expenditures among Individuals with T2DM}

Randomized clinical trials that have studied whether among individuals with both depression and T2DM, depression treatment delivered in primary care based collaborative care settings (where both depression and T2DM is managed with the help of coordinated healthcare teams comprising of primary care physicians, nurses and other specialists) results in reduction of healthcare expenditures as compared to usual care. The Improving Mood-Promoting Access to 
Collaborative (IMPACT) randomized controlled trial included 418 individuals aged 60 years or older, of them 204 received the collaborative care intervention and 214 were randomized to usual care; this study found that at the end of 24 months the intervention group had \$896 lower expenditures as compared to the usual care group [8]. The Pathways study $[9,10]$ was also a randomized controlled trial which was carried out in 9 primary care settings across Washington and Idaho; the participants were members of a mixed model health plan. The intervention provided was a 12 month stepped-care depression management program delivered via primary care physicians, registered nurses who received training in problem solving therapy for depression and psychiatrists. Depression treatment for intervention group (mean age 58 years) in step 1 started with antidepressant drugs or psychotherapy, in case of no response to treatment in step 1 , treatment modality was adjusted in step 2 , e.g. those treated with antidepressants in step 1 , were given psychotherapy in step 2 , in case of no response to treatment in step 2 , treatment in step 3 involved referral to specialist; the control group (mean age 57 years) was given usual care. Total healthcare expenditures reported at the end of 2-year and 5-year periods were found to be lower in the intervention group as compared to control group: 2 year mean (SD) in intervention vs control group were $\$ 21,148$ ( $\$ 27548)$ vs $\$ 22,258(\$ 35,607)$ [9] and 5 year mean(SD) in intervention vs control group were 49,254 (50,773) vs 49,254 (50,773) [10].

Studies examining the association between depression treatment with healthcare expenditures among individuals with coexisting T2DM and depression are very limited. So, evidence from studies examining the association between depression treatment and healthcare expenditures among individuals with other chronic conditions is discussed. Using administrative claims data, one study showed that among individuals with dyslipidemia, T2DM and coronary artery disease, either in combination or alone, antidepressant medication adherence improved 
adherence to coexisting disease medications and thus reduced one-year healthcare expenditures [11]. On the contrary, one study carried out with real world data from Medicare Current Beneficiary Survey (MCBS),found that among beneficiaries with coexisting depression and chronic diseases such as arthritis, diabetes, respiratory diseases including asthma, chronic obstructive pulmonary disease, heart disease, hypertension, osteoporosis, and stroke, those receiving depression treatment had higher inpatient, medical provider and prescription drug expenditures [12]; however, this study was restricted to the elderly population.

While randomized controlled trials have demonstrated that depression treatment delivered in collaborative care settings reduced expenditures among individuals with T2DM and coexisting depression, studies conducted using real world observational data have reported inconsistent findings. However these studies were not specific to T2DM population and included special populations such as elderly. Therefore, it remains to be established whether depression treatment with antidepressants and psychotherapy, alone or in combination, are associated with reductions in healthcare expenditures, among individuals with T2DM. Based on evidence from depression care delivered in collaborative care settings, one may infer that combined antidepressants and psychotherapy may reduce healthcare expenditures.

\section{The Relationship between Depression Treatment and Healthcare Expenditures by Coexisting}

\section{Chronic Physical Conditions}

Virtually, there are no existing studies using real world data that are specific to populations with coexisting T2DM and depression which have examined the association between depression treatment and healthcare expenditures; therefore there is no knowledge on whether this association varies by types of coexisting chronic physical conditions. Findings from unpublished studies conducted by the authors of this study, have revealed that among individuals 
with T2DM, the risk of having newly-diagnosed depression and treatment received by those with newly-diagnosed depression varied by types of coexisting chronic physical conditions.

Therefore, it is highly plausible that healthcare expenditures following depression treatment may also be associated with coexisting chronic physical conditions. With majority of adults $(88.6 \%)$ with T2DM in the US having at least one chronic condition and 15\% having reported four or more chronic conditions [13], presence of other coexisting chronic physical conditions among individuals with T2DM is a norm rather than an exception. Therefore, in absence of any related literature it is important to examine whether the relationship between depression treatment and expenditures vary by types of coexisting chronic physical conditions.

\section{Need for the study}

As explained above there is a significant gap in the literature on whether depression treatment can reduce healthcare expenditures among individuals with T2DM. To the best of the authors' knowledge, no study has examined whether the association between depression treatment and healthcare expenditures among individuals with T2DM vary by types of coexisting chronic physical conditions.

It is particularly important to study this association in non-elderly population given that, in recent years, the prevalence of multiple coexisting chronic conditions, which has traditionally been thought of as a problem in the elderly population, has been increasing among non-elderly adults $[14,15]$. In the US, among adults participating in the national health interview survey and in the age group 45-64 years, the prevalence of multiple coexisting chronic conditions has been increasing and has grown from $24.5 \%$ in 2004 to $28.1 \%$ in 2010 [16]. As risk of depression increases with increasing number of coexisting chronic physical conditions [17], there is a need to evaluate the impact of type of depression treatment on healthcare expenditures and whether 
this relationship vary by types of coexisting chronic physical conditions among non-elderly adults aged 18-64 years.

In the US, state Medicaid plans are a major provider of health insurance among nonelderly adults. As of 2010, 27\% of US adults, 20-64 years of age were covered by Medicaid, outlaying $68 \%$ of the federal spending [18]. Adults with diabetes are disproportionately covered by Medicaid and four out of five Medicaid beneficiaries with diabetes suffer from a coexisting chronic physical condition. It has been reported that among adults enrolled in Medicaid, those with diabetes spend more than 2.5 times compared to those without diabetes [19]. Twenty percent of Medicaid enrollees suffer from depression [20, 21].According to Agency of Healthcare Research and Quality 2011 statistical brief, mood disorders which includes depression ranks top most among the ten conditions for 30 day readmission in Medicaid resulting in 41,600 readmissions and costing \$286 million [22]. Depression is also a major driver for poor health outcomes and future healthcare expenditures in T2DM [23]. Given such compelling statistics, it is apparent that Medicaid data provides a rich opportunity to conduct research of both depression and T2DM, and can lead to important findings that might influence clinical practice and policy. Therefore, among the high risk population of Medicaid enrollees with T2DM, it is necessary to delineate the relationship between depression treatment and healthcare expenditures and understand how types of coexisting chronic physical conditions impact this association. 


\section{RESEARCH OBJECTIVES AND HYPOTHESES}

Aim 3.1: Among non-elderly Medicaid beneficiaries with T2DM and newly-diagnosed depression, examine the association depression treatment categories and total and T2DM-related healthcare care expenditures.

Hypothesis 3.1: As compared to those with no depression treatment, those with depression treatment with only antidepressants, only psychotherapy or both, will have negative associations with total and T2DM-related healthcare expenditures as compared no depression treatment. Aim 3.2: Among non-elderly Medicaid beneficiaries with T2DM and newly-diagnosed depression, examine whether the association between depression treatment and total and T2DMrelated medical care expenditures vary by types of coexisting chronic physical conditions. Hypothesis 3.2.1: As compared to no depression treatment, treatment within both antidepressants and psychotherapy will reduce total and T2DM related healthcare expenditures across all conditions.

Hypothesis 3.2.2: Depression treatment with only antidepressants will not be associated with T2DM related healthcare expenditures among those with concordant conditions only. 


\section{CONCEPTUAL FRAMEWORK}

The basic framework used in this study was a hybrid of the expanded behavioral model on use of health services, widely known as the Andersen behavioral model (ABM) [24]. The ABM posits that utilization of health services varies as a function of 1) each individual's unique predisposition for using services (predisposing factors); 2) the means available to each individual for obtaining services (enabling factors); 3) each individual's level of need; 4) personal health practices; and 5) the external environment (figure 1). The behavioral health model is very flexible and was easily adapted to analyze the relationship between depression treatment and types of coexisting chronic physical conditions and the association between depression treatment and healthcare expenditures.

\section{Predisposing factors: \\ Gender, age, race/ethnicity}

Enabling factors:

Medicaid Eligibility

\section{Need Factors:}

Types of coexisting physical conditions, Other Mental Health Conditions, Number of chronic conditions

\section{External Environment:}

e.g. County level social determinants of health County Median Income, State, \% with college education and \% below poverty level and Community level healthcare infrastructure e.g. presence of $\mathrm{CMHC}, \mathrm{FQHC}$ in a county

\section{Personal Health Practices:}

e.g Health care Seeking Behavior: Medication use, use of inpatient and emergency room services
Aim 3 - Dependent Variables:

Total Healthcare Expenditure T2DM-Related Healthcare Expenditure 


\section{METHODS}

\section{Study Design}

A retrospective longitudinal study repeated measures cohort design was used. The study cohort included individuals with T2DM and newly-diagnosed depression. "Index date" was defined as the first date of depression diagnosis and baseline and follow-up periods were respectively defined as 12 months prior and subsequent to this index date. Inpatient, outpatient and prescription drug expenditures were identified at the end of each month of follow-up. Therefore, each individual included in the study cohort had 12 observations. Appropriate statistical models were used to account for such repeated measures design. Independent measures design, which often use aggregate expenditures at the end of follow-up period, do not capture the variation in healthcare expenditures at different time points of follow-up. The repeated measures analysis helped in capturing healthcare expenditures both during and after the acute phase of depression treatment.

\section{Data Source}

Medicaid analytic extract (MAX) files:

The MAX files are prepared and produced by the Centers for Medicare and Medicaid Services. Person-level data such as eligibility, demographics, managed care enrollment, a utilization summary and Medicaid payments for enrollees are provided in the enrollment ("personal summary") file. Information on International Classification of Diseases $9^{\text {th }}$ revision (ICD-9-CM codes) of conditions diagnosed, healthcare service utilizations and charges paid by Medicaid for the services can be extracted from inpatient, other therapy files. The pharmacy file provides information on variables such as national drug code (NDC) of medications used and 
amount paid by Medicaid for the medication claims. This study used Medicaid data from 20002008 data from three states: New York (NY), Texas (TX), Illinois (IL).

Area Health Resource File (AHRF):

The AHRF is national county-level health resource information provided by the U.S. Department of Health and Human Services (2011) that provides information on more than 6,000 variables county level variables such as characteristics of health facilities, number and type of health professionals in a county, rural-urban status, resource scarcity, health status, economic activity, health training programs, and socioeconomic and environmental characteristics [25]. The Federal Information Processing Standard (FIPS) county codes which are available both in the AHRF file and the personal summary file of the MAX data, were used to link the two files.

\section{Identification of T2DM and Newly-diagnosed Depression Cohort}

Three state Medicaid data from 2000-2008 was used to identify seven longitudinal cohorts: 2000-02, 2001-03, 2002-04, 2003-05, 2004-06,2005-07, 2006-08.

\section{Medicaid Beneficiaries with T2DM:}

Medicaid enrollees with at least one inpatient visit or two or more physician outpatient visits which were at least 30 days apart, with a primary or secondary diagnosis of ICD-9-CM codes: 250.x0 or 250.x2 during a calendar year, were identified as having T2DM. Medicaid beneficiaries with T2DM who had a diagnosis of depression or antidepressant medication use during the calendar year were excluded.

\section{Medicaid Beneficiaries with Newly-diagnosed Depression:}

The eligible study population was followed into the subsequent calendar year to identify newly-diagnosed depression. Enrollees with at least one outpatient physician visit or an inpatient admission with a primary or secondary diagnosis of depression in the following calendar year 
were classified as having a newly-diagnosed depression [26]. Depression was identified using ICD9 CM codes: 296.2 (major depressive disorder, single episode), 296.3 (major depressive disorder, recurrent episode), 311 (depression not elsewhere classified), 309.1 (prolonged depressive reaction), 300.4 (neurotic depression) and 298.0 (depressive type psychosis). These ICD-9-CM codes are extensively used by health plans to identify depression [27] and have also been used in previous literature that have studied depression in Medicaid enrollees [28-30]. Those with no newly-diagnosed depression were excluded from the study cohort. The first observed date of outpatient visit or inpatient discharge with diagnosis of depression was the "index date"; 365 days prior to the index date was required to be free of any depression diagnosis or antidepressant medication. One may note that other studies have 120 day depression free period to define newly-diagnosed depression [31]. However, the 365 day look-back period was used with the intent to minimize misclassification of an episodic manifestation of chronic depression (where depression symptoms last for two or more years) as newly-diagnosed depression.

Additional exclusion criteria were (1) not having a diagnosis of at least one chronic physical condition (identified by ICD-9-CM codes included in the appendix) in the baseline period (2) no continuous fee-for-service Medicaid eligibility and (3) enrollment in Medicare at any point during the observation period; (4) died during the study period and (5) did not use inpatient or outpatient Medicaid services during the study period.

\section{Dependent Variables}

Total Healthcare Expenditures:

Total healthcare expenditure per person included in the study cohort was defined as the total dollar amount that Medicaid paid for inpatient, outpatient and pharmacy claims. Total 
healthcare expenditures were identified at the end of each month of follow-up during the 12 month follow-up period. The total healthcare expenditures were adjusted by the medical component of the Consumer Price Index (CPI) and expressed in the 2008 constant dollars. After assessing the skewness and kurtosis properties and linearity through qq-plots, the total health expenditures were log transformed and used as dependent variable.

\section{T2DM-Related Healthcare Expenditures:}

T2DM-related healthcare expenditure per person included in the study cohort were defined as the total dollar amount that Medicaid paid for inpatient and outpatient claims with a diagnosis of T2DM. The T2DM-related healthcare expenditures were also identified on a monthly basis during the 12 month follow-up period. Similar to total healthcare expenditures, the T2DM-related expenditures were also adjusted by the medical component of the CPI and expressed in the 2008 constant dollars and were log transformed.

\section{Independent Variables}

Key Independent Variables:

Depression treatment during the acute phase: The first four months following the newlydiagnosed depression is known as the acute phase of depression treatment. The acute phase is used to set treatment goals, assess risk of suicide, decide on using psychotherapy and/or appropriate antidepressant, enhance adherence and monitor response [32]. The initial treatment choice may influence the effectiveness of depression treatment and therefore may also be associated with healthcare expenditures over time.

Depression treatment in the acute phase could be provided with only antidepressants or only psychotherapy or both. Antidepressant Use: The national drug codes available in the prescription drug use file of MAX data were used to identify antidepressant drug classes: 
selective serotonin reuptake inhibitors, selective norepinephrine reuptake inhibitors, tricyclic antidepressants, monoamine oxidase inhibitors and other (mirtazapine and bupropion). Psychotherapy Use: The use of psychotherapy was identified with Current Procedural Terminology (CPT) codes. The following psychotherapy types were used: (i) psychotherapy diagnostic interview $(90801,90802)$ (ii) individual psychotherapy [individual psychotherapy 20$30 \min (90804,90816,90805,90817), 45-50 \min (90806,90818,90807,90819), 75-80 \min$ (90808, 90821, 90809, 90822); interactive individual psychotherapy 20-30 $\min (90810,90823$, 90811, 90824), 45-50 $\min (90812,90826,90813,90827), 75-80 \min (90814,90828,90815$, 90829)] (iii) other psychotherapy [family psychotherapy (90846, 90847, 90849), group psychotherapy (90853), interactive group psychotherapy(90857)][33].

Depression treatment in the acute phase was categorized as treatment with: (1) Only Antidepressants: These individuals received at least one prescription for antidepressant, but no psychotherapy visit during the acute phase of depression treatment were considered as being treated with only antidepressants; (2) Only Psychotherapy: Those who received at least one psychotherapy office visit, but no prescription for antidepressant drugs during the acute phase were considered as being treated with only psychotherapy. (3) Both Antidepressants and Psychotherapy: Those who received a minimum of one prescription for antidepressants and one psychotherapy visit during the first120 days following newly-diagnosed depression were considered as being treated with both antidepressants and psychotherapy (4) No treatment: These individuals received no prescriptions for antidepressants and no psychotherapy office visit during the acute phase.

Types of Coexisting Chronic Physical Conditions (Need Factor used in ABM): 
As other physical conditions coexisting with T2DM often impact the medical care, selfmanagement, and healthcare outcomes of an individual with T2DM, Piette and Kerr developed a framework that classified coexisting conditions among individuals with T2DM into categories based on similarities and differences from T2DM pathophysiology and management. The categories of conditions that might coexist with T2DM were defined as: dominant (conditions whose severity eclipses all other conditions' management plans such as metastatic cancer), concordant (conditions that overlap with T2DM in their pathophysiology and management plans such as cardiovascular diseases) or discordant (conditions with unrelated pathophysiology or management plans such as musculoskeletal disorders) [34].

Following this framework, one may assume that presence of such coexisting conditions among individuals with T2DM and newly-diagnosed depression may also affect the management of depression and therefore response to depression treatment. Therefore, based on this theoretical framework, forty-four different types of coexisting chronic physical conditions a list of which along with the ICD-9-CM codes used to identify the conditions is presented in Appendix A, were used to define types of coexisting chronic physical conditions. A hierarchical classification was followed [23]; dominant conditions were given priority because such conditions often take precedence over the management of other health conditions. Only among those without dominant conditions, concordant and discordant conditions were identified. The types of coexisting chronic physical conditions were classified as: 1) Dominant conditions 2) Concordant only, 3) Discordant only and 4) Both concordant and discordant.

\section{Other independent variables:}

Based on the Andersen's Behavioral Model, the other independent variables included in the study were: 
Predisposing Factors: The included variables were: demographics (gender, age, race/ethnicity [Whites, African Americans, Hispanics or other races]).

Enabling Factors: Using Medicaid eligibility status the enabling factors included were: eligibility due to poverty (yes/no), medical needs (yes/no) and waiver (yes/no). Need Factors: The variables included were clinical characteristics such as presence of other mental health conditions and number of conditions during baseline. The other mental health conditions included bipolar disorder, schizophrenia, post-traumatic stress disorder, and alcohol and drug abuse.

Personal Health Practices: As an individual's propensity to seek treatment may influence healthcare utilizations and thereby expenditure, therefore the study controlled for healthcare seeking behavior by controlling for baseline healthcare utilization characteristics such as number of emergency room visits in 180 days prior to new-onset depression, inpatient hospitalization, number of oral antidiabetic medication classes and insulin use as identified by NDC codes, presence of polypharmacy identified by use of 6 or more drug classes in the 90 days prior to new-onset depression, number of outpatient visits measured in quartiles and total baseline healthcare expenditures

External Environment: External environment variables included state of residence, community level access healthcare infrastructure and community level social determinants of health variables. Community level access healthcare infrastructure variables included presence of community mental health clinic (CMHC) and federally qualified health clinic (FQHC) in a county, whether county of residence was designated as Health Professional Shortage area (HPSA) and density of social workers in a county. The social determinants of health variables included were urban/rural status of a county, median income in the county and whether percent 
below poverty level and percent with college education in the county were greater than national average based on US census estimates [35, 36].

Other variables: After response to depression treatment is achieved in the acute phase, treatment for depression may be continued for another 4 to 9 months of continuation phase treatment [37]. Therefore, it may be plausible that some individuals received depression treatment with antidepressants and/or psychotherapy during entire length of follow-up and some did not. To control for such variation, the statistical models additionally controlled for antidepressant treatment at each month of follow-up: This was defined as a dichotomous (yes/no) variable, which indicated whether an individual received antidepressant prescription during each month of the follow-up; psychotherapy treatment at each month of follow-up: A categorical (yes/no) variable that indicated whether an individual received outpatient psychotherapy during each month of the follow-up. year of observation: As data from multiple years forming seven different panels (2000-2002, 2001-2003, 2002-2004, 2003-2005, 2004-2006, 2005-2007, 20062008) were used, a variable indicating the particular cohort the observation came from was also included.

\section{Statistical Analyses}

Bivariate Analyses:

Unadjusted analyses using descriptive statistics, including frequency, percentage, mean, and standard errors were conducted. Chi-square analyses were used to examine unadjusted differences in baseline characteristics and types of coexisting chronic physical conditions. Chisquare analyses were used to examine unadjusted differences in types of coexisting chronic physical conditions and depression treatment.

Analyses with repeated measures: 
As healthcare expenditures were aggregated for each month of follow-up, 12 observations were available for each individual. Due to repeated measures of healthcare expenditures, the observations were not independent. Because standard regression techniques assume that individual observations are independent, they cannot be applied to data with repeated measures. More appropriate models are those that account, for such dependence. Therefore, the multivariable analyses consisted of linear mixed effects models. These models account for correlated error terms of observations from same person.

Linear mixed model analyses were conducted to assess the association between depression treatment categories during the acute phase and total and T2DM-related healthcare expenditures. The statistical model included the key independent variable i.e. depression treatment categories during the acute phase, types of coexisting chronic physical conditions, and predisposing, enabling, need, personal health practices (health seeking behavior), external environment characteristics and other variables (antidepressant treatment at each month of follow-up, psychotherapy treatment at each month of follow-up, year of observation) . Additionally, separate linear mixed model regressions for each type of coexisting chronic physical conditions were conducted to examine whether the association between depression treatment and total and T2DM-related healthcare expenditures vary by types of coexisting chronic physical conditions.

Observed Selection Bias: Adjusting for inverse probability weighting technique (IPTW)

Depression treatment is a choice variable and observed differences in individual as characteristics of the study population can influence this choice. To account for such observed differences the inverse probability weighting technique (IPTW) was used. The IPTW gives weight to each individual based on the inverse of their propensity to use a particular type of 
depression treatment. Thus individuals who have lower propensity are up-weighted and those with higher propensity are down weighted. This helps to balance the probability of treatment across the treatment groups. In order to account for the differences in group sizes of the treatment groups, the weights were further stabilized by dividing them with sample size of each group.

For easier interpretation of log transformed expenditure variables, percentage changes in total and T2DM-related expenditures for depression treatment categories were calculated by subtracting one (depression treatment being categorical) from exponentiated beta coefficients and multiplying this number by 100. All analyses were conducted using Statistical Analysis Software (SAS 9.3).

\section{Secondary Analysis}

It has been observed the individuals with depression often do not get adequately treated owing to various clinician and patient related factors such as short duration of treatment and noncompliance to medication use [38]. However, adequate depression treatment has been found to be associated with lesser increase in healthcare expenditures from pre-depression to postdepression time periods as compared those not adequately treated, especially in presence of other coexisting chronic conditions [39]. Therefore, this study additionally examined whether adequate depression treatment in the acute phase was associated with healthcare expenditures in this study population. Results of this analysis are presented in Appendix B. 


\section{RESULTS}

\section{Description of the Study Population}

The study population consisted of 5,295 non-elderly fee-for-service Medicaid beneficiaries with T2DM and newly-diagnosed depression. Description of the study population is presented in Table 1. In the study population, $36.3 \%$ were aged $45-54$ years and $38.5 \%$ were older adults aged between 55 and 64 years, $67.3 \%$ were females; $26.8 \%$ were Whites and $30.1 \%$ were African Americans and $43 \%$ belonged to other races; majority of the counties of residence were metro areas $(89.1 \%)$, however, $83.2 \%$ of counties were designated as shortage area for mental health professionals and $51.1 \%$ of counties did not have a CMHC.

\section{Description of the Study Population by Types of Coexisting Chronic Physical conditions}

Table 1 also presents a description of the study population by types of coexisting chronic physical conditions. In the study population, $14.2 \%$ had dominant conditions, $27.3 \%$ had concordant conditions only, $15.8 \%$ had discordant conditions only and $42.8 \%$ had both concordant and discordant conditions. All individual baseline characteristics and majority of the county level characteristics differed significantly among the types of coexisting chronic physical conditions groups. For example, a greater proportion of individuals with dominant conditions were older i.e.55-64 years (17.4\% vs $9.1 \%$ in $18-44$ years age-group), males (18.3\% vs $12.3 \%$ females), had presence of other mental health conditions (18.6\% vs $11.6 \%$ with absence of other mental health conditions), inpatient hospitalizations (20.5\% vs $8.1 \%$ in those without) and higher number of outpatient visits $(22.7 \%$ in 4 th vs $6.1 \%$ in 1 st quartile). For the external environment characteristics, presence of a CMHC in the county and percentage of the county population below the national poverty level of $11.1 \%$ were the two variables that did not significantly differ among the types of coexisting chronic physical condition subgroups. 


\section{Unadjusted Association between Types of Coexisting Chronic Physical Conditions and Depression Treatment Categories}

During the acute phase, $27.3 \%$ had treatment with only antidepressants, $18.1 \%$ had treatment with only psychotherapy, $11.4 \%$ had treatment with both antidepressants and psychotherapy and $43.2 \%$ of the study population had no depression treatment. Unadjusted chisquare analyses revealed that depression treatment during the acute phase varied significantly (Pvalue $<0.001$ ) among the types of coexisting chronic physical conditions subgroups. A significantly lower percentage of individuals with dominant conditions (19.1\%) and concordant conditions only (27.3\%) had treatment with only antidepressants as compared to those with discordant conditions only (32.7\%); a significantly higher percentage of individuals in dominant conditions (28.2\%) and concordant conditions only (17.2\%) received treatment with only psychotherapy as compared to individuals with discordant conditions only (14.0\%); treatment with both antidepressants and psychotherapy was received by $12.6 \%$ with dominant conditions, $11.2 \%$ with concordant conditions only, $10.7 \%$ with discordant conditions only and $11.4 \%$ with both concordant and discordant conditions. The percentages with no depression treatment were $40.1 \%$ for those with dominant conditions, $44.3 \%$ for individuals with concordant conditions only, $42.6 \%$ for individuals with discordant conditions only and $43.8 \%$ for individuals with both concordant and discordant conditions. Results are not presented in tabular form.

\section{Mean Monthly Healthcare Expenditures for Depression Treatment Categories}

Total Healthcare Expenditures: The mean total healthcare expenditures for 12 months after diagnosis of depression were $\$ 30,590$ for those treated with only antidepressants, $\$ 35,099$ for those treated with only with psychotherapy, $\$ 33,032$ for those treated with both antidepressants and psychotherapy and $\$ 34,041$ for those receiving no depression treatment. The 
mean monthly expenditures across all depression treatment categories decreased over time. For example, in the antidepressant only group, the mean expenditure reduced from $\$ 4,729$ at the end of Month 1 to $\$ 2,497$ at the end of Month 12; for the only psychotherapy group the reduction from Month 1 to Month 12 was $\$ 6,172$ to $\$ 2,621$; for those with both antidepressant and psychotherapy treat the mean expenditures reduced from \$5,473 (Month 1) to \$2,327 (Month 12) and among those with no treatment the total healthcare expenditures reduced from \$5291 (Month 1) to $\$ 2,689$ (Month 12).

T2DM-related Healthcare Expenditures: The mean total healthcare expenditures for 12 months after diagnosis of depression were $\$ 13,642$ for those treated with only antidepressants, $\$ 15,654$ for those treated with only psychotherapy, $\$ 15,726$ for those treated with both antidepressants and psychotherapy and $\$ 14,801$ for those receiving no depression treatment. Similar to the reductions observed in total healthcare expenditures over each month, the reduction in T2DM-related healthcare expenditures from Month 1 to Month 12 was $\$ 2,848$ to $\$ 895$ for only antidepressants, $\$ 3,419$ to $\$ 1,013$ for only psychotherapy , $\$ 3176$ to $\$ 768$ for those treated with both antidepressants and psychotherapy and $\$ 3,305$ to $\$ 1,065$ for those received no depression treatment. Whether the mean monthly expenditures

The total and T2DM-related healthcare expenditures aggregated at ends of Months1-4, Months 5-8 and Months 7-12 are presented in Figure1.

Mean Monthly Healthcare Expenditures for Depression Treatment Categories: by Types of Coexisting Chronic Physical Conditions

Total Healthcare Expenditures: Total healthcare expenditures reduced with in each subgroup of types of coexisting chronic physical conditions. 
T2DM-related Healthcare Expenditures: Similarly, The T2DM-related healthcare expenditures also reduced with in each subgroup of types of coexisting chronic physical conditions.

Total and T2DM-related healthcare expenditures aggregated at ends of Months1-4, Months 5-8 and Months 9-12 by each subgroup of types of coexisting chronic physical conditions are presented in Figure 2.

Adjusted Association between Depression Treatment Categories during the Acute Phase and Healthcare Expenditures

Total Healthcare Expenditures: Linear mixed model regression analyses were conducted to examine the association between depression treatment categories and log transformed total and T2DM-related healthcare expenditures. In Model 1, which did not include the interaction term between depression treatment categories and types of coexisting chronic physical condition, it was found that, as compared to no treatment, all other types of treatment was associated with reduction in total healthcare expenditures. As compared to no depression treatment, depression treatment with only antidepressants was associated with $17 \%$ (beta: -0.18 ; SE: 0.04$)$ reduction in total healthcare expenditures, treatment with only psychotherapy was associated with $22 \%$ (beta: -0.25 ; SE: 0.05 ) reduction in total healthcare expenditures and treatment with both antidepressants and psychotherapy was associated with $28 \%$ (beta: -0.33 ; SE: 0.06$)$ reductions in total healthcare expenditures.

T2DM-related Healthcare Expenditures: Treatment with only antidepressants was not significantly associated with reduced log transformed T2DM-related expenditures as compared to no depression treatment. However, treatment with psychotherapy and both antidepressants and 
psychotherapy was associated with $28 \%$ and $18 \%$ reduction in T2DM-related expenditures as compared to no depression treatment.

The results are presented in Table 3.

\section{Adjusted Association between Depression Treatment Categories during the Acute Phase and Healthcare Expenditures by Types of Coexisting Chronic Physical Conditions}

Total Healthcare Expenditures: Treatment with only antidepressants was associated with $21 \%$ (beta: -0.24 ; SE: 0.18 ) reductions in total healthcare expenditures among those with concordant conditions only and by $18 \%$ (beta: -0.20 ; SE: 0.09 ) among those with discordant conditions only. Depression treatment with only psychotherapy was associated with 48\% (beta: 0.65; SE: 0.12) reductions in total healthcare expenditures among those with dominant conditions and $21 \%$ (beta: -0.23 ; SE: 0.07 ) reductions in total healthcare expenditures among those with both concordant and discordant conditions. Treatment with both antidepressants and psychotherapy was associated with reductions in total healthcare expenditures among all types of coexisting chronic physical condition groups.

T2DM-related Healthcare Expenditures: Associations with reduced T2DM-related healthcare expenditures were not observed with treatment by only antidepressants. Treatment with only psychotherapy was associated with $41 \%$ (beta:-0.53; SE: 0.14 ) reduction in T2DMrelated healthcare expenditures among those with dominant conditions and 34\% (beta:-0.41; SE: 10) reduction in T2DM-related healthcare expenditures among individuals with both concordant and discordant conditions. Treatment with both antidepressants and psychotherapy was associated with 27\% (beta:-0.32; SE: 0.13) reduction in T2DM-related healthcare expenditures among those with concordant conditions only.

Table 4 presents the beta-coefficients and standard errors 


\section{DISCUSSION}

This study examined the association between depression treatment categories and total and T2DM-related healthcare expenditures and further assessed whether this association varied by types of coexisting chronic physical conditions among non-elderly Medicaid beneficiaries with T2DM and newly-diagnosed depression. Multiple unique significant findings were observed.

\section{Depression Treatment Categories during the Acute Phase and Healthcare Expenditures}

- In this study population, $43 \%$, or 4 in 10 i.e individuals with T2DM and newly diagnosed depression did not receive any treatment for depression.

- However, it was observed that as compared to not receiving treatment for depression, any modality of depression treatment reduced both total healthcare expenditures.

- With regards to T2DM-related expenditures, the modality of depression treatment was important; treating depression with only antidepressants did not reduce T2DM-related healthcare expenditures.

Total Healthcare Expenditures: The study findings indicate that treating depression is associated with a reductions in total healthcare expenditure. There are some plausible explanations for such an observed association between depression treatment and reduced healthcare expenditures. Randomized Clinical trials which have examined the association of depression treatment with pharmacotherapy i.e. antidepressants and/or psychotherapy have found that depression treatment with antidepressants reduced depressive symptoms among individuals with T2DM and depression [40-47] and depression treatment with psychotherapy such as cognitive behavioral therapy was also effective in reducing depression symptoms [48-50]. Therefore, treating depression may reduce healthcare expenditures by providing relief from 
depressive symptoms by reducing mental-health related expenditures. Additionally, individuals with depression have been shown to have high utilizations of healthcare services $[51,52]$ and also there is evidence that coexisting depression worsens of other medical conditions by its adverse effect of medication adherence [53] and self-care regimens [54]. Treatment of depression may result in subsequent relief from symptoms and therefore, may result in reduced healthcare service utilizations, improved adherence of other chronic disease medications and better self-care, thereby, reducing healthcare expenditures.

T2DM-related Healthcare Expenditures: An interesting finding was that, depression treatment with only antidepressants did not reduce T2DM-related healthcare expenditures. This finding can be explained by the fact that among individuals with T2DM and depression, clinical trials of depression treatment with only antidepressants did not provide conclusive evidence of better T2DM related outcomes such as glycemic control [55]. Furthermore, among adults with T2DM, depression treatment with antidepressants can increase cardiovascular morbidity and mortality $[56,57]$. Therefore, among individuals with T2DM, treatment with antidepressants may not be a suitable option for reducing T2DM-related expenditures.

However, depression treatments modalities which included which psychotherapy was a component of treatment was associated with reductions in T2DM-related healthcare expenditures. This finding is comparable to the limited evidence from previous research. For example, Lustman et al., showed that treatment of depression among 51 individuals with T2DM and depression, 10 weeks of cognitive behavioral therapy was not significantly associated with reduced glycated hemoglobin (A1C) levels in the intervention group at 6 month after follow-up (intervention vs control: $9.5 \%$ vs $10.9 \% ; \mathrm{P}=0.03$ ). No study in real-world setting has examined the effects of treating depression with both antidepressants and psychotherapy. However, 
evidence from randomized controlled trials of collaborative care which includes often depression treatment with antidepressants as well as psychotherapy (either initiated together or in a stepped care approach based on response to initial treatment) have shown significant reductions in A1C levels in intervention as compared to control group [58]. Such evidence, supports this study findings that treatment of depression with psychotherapy can reduce T2DM-related expenditures. Depression Treatment Categories during the Acute Phase and Healthcare Expenditures: By Types of Coexisting Chronic Physical Conditions

- Across all types of co-existing conditions treatment for depression with combination therapy (i.e. antidepressants and psychotherapy) was associated with reduced total healthcare expenditures.

- The relationships between other depression treatment categories and total healthcare expenditures varied by types of coexisting chronic physical conditions.

- For some conditions treatment for depression with only antidepressants reduced total healthcare expenditures. These were: concordant conditions only and discordant conditions only

- For other conditions, treatment for depression with only psychotherapy reduced total healthcare expenditures: these conditions were: dominant conditions and both concordant and discordant conditions.

- The associations between depression treatment categories and T2DM-related expenditures varied by types of co-existing chronic conditions. For some conditions psychotherapy reduced T2DM-related expenditures: these were dominant conditions and both concordant and discordant conditions; for 
concordant only condition combined antidepressants and psychotherapy reduced T2DM-related expenditures.

Taken together these findings highlight the importance of combined antidepressants and psychotherapy as the best alternative to reducing total healthcare expenditures among individuals with T2DM and newly diagnosed depression. Several studies, including multiple randomized clinical trials [59-61] and meta-analyses $[62,63]$ have shown that, among individuals with depression, combined treatment with both pharmacotherapy and psychotherapy significantly reduces depression symptoms and dropout rates. Treatment with both antidepressants and psychotherapy has also been found to found to have long term benefits in terms of preventing relapse [67] and increasing depression treatment adherence [68-70]. Therefore, by improving depression related outcomes, treatment with both antidepressants and psychotherapy may help to reduce total healthcare expenditures.

However, if physicians or patients prefer treatment either with antidepressants or psychotherapy alone, then the choice of treatment need to be prioritized based on type of coexisting chronic physical condition. Depression treatment with only psychotherapy reduced total healthcare expenditures among those with high burden of coexisting conditions such as those with dominant conditions and also individuals with both concordant and discordant conditions; additionally, it also needs to be noted that, treatment with only psychotherapy also reduced T2DM-related healthcare expenditures among these individuals. Individuals with dominant and those with both concordant and discordant conditions may have higher disease burden and treating depression in such patients can be challenging. A previous study has shown that principles of certain types of psychotherapy, such as cognitive behavioral therapy may help in managing aspects of chronic physical conditions such as pain $[64,65]$. Pain management is an 
integral part of dominant conditions such as cancer and multiple sclerosis. Presence of both concordant and discordant conditions, for example, coexisting neuropathy and rheumatoid arthritis, may also result in exacerbated pain. Psychotherapy such as cognitive behavioral therapy has also been proven to be beneficial for management of pain $[66,67]$. Treatment with psychotherapy, in such cases, may result in more holistic treatment and better outcomes as seen by reduced healthcare expenditures as compared to treatment with only antidepressants.

\section{Implications}

The study findings have significant health policy implications. In this study expenditures over a one year period were examined among Medicaid beneficiaries with newly-diagnosed depression. Therefore, this could be viewed as expenditures following a new episode of depression in a high risk group of Medicaid beneficiaries with T2DM and coexisting conditions. In general, the study results indicated that depression treatment is associated with reduced healthcare expenditures, however the association varies by types of coexisting chronic physical conditions. Such results have important implications in the context of new payment models such as the "bundled payment" where providers or facilities are paid a single payment for all services in relation to treating a condition or provide a treatment. The results of this study indicated the some types of depression treatment is no better than not treating depression at all, in terms of association with reduced healthcare expenditure. For example, depression treatment with only antidepressants in presence of dominant conditions is not associated with reduced healthcare expenditures. Therefore, among individuals with T2DM and coexisting dominant conditions, physicians may not want to initiate treatment for newly-diagnosed depression with antidepressants, under the bundled payment systems.

The findings also have implications for improving quality of care initiatives such as Medicare Shared Savings Program (MSSP). The MSSP [68] use expenditure patterns of 
Medicare beneficiaries in the past three years to set expenditures "benchmarks", using risk adjustment models. These benchmarks are used to establish savings and share the cost-reduction with the CMS. [69] Although, the MSSP is specific to Medicare, many state Medicaid agencies are using the MSSP as one of the models for building their accountable care organization (ACO) programs. The study results have implications with regards to such "benchmarking" approaches. Response to depression treatment in terms of economic benefits varied by types of coexisting conditions present. Therefore, total healthcare expenditures among Medicaid beneficiaries with T2DM and newly-diagnosed depression and coexisting conditions may vary both by types of coexisting conditions and types of depression treatment received. Therefore, while setting benchmarks for individuals with T2DM, newly-diagnosed depression and other coexisting chronic physical conditions, one will need to risk adjust for type of coexisting conditions and types of depression treatment categories. .

The study findings further suggests that economic benefits of depression treatment may be more achievable in integrated care settings where physical and mental healthcare are delivered in tandem and treatment of depression is adjusted as per individual response. The 2010 Patient Protection and Affordable Care Act aims to promote such integrated care through patient centered medical homes (PCMH). For managing treatment of mental health conditions such as depression, the PCMH model promotes coordinated care among primary care physicians, a mental healthcare specialist and a care manager [70]. As care is integrated, primary care physicians can monitor the overall health and can easily consult and refer with mental healthcare specialists and thus provide more holistic care to individuals with both depression and multiple coexisting chronic physical conditions [70]. As of 2013, 43 of 50 states of US had adopted policies to promote PCMH [71]. Therefore, in coming years, studies need to be conducted to 
assess depression treatment patterns and associated long term expenditures among individuals with coexisting depression and multiple chronic physical conditions.

\section{Strengths and Limitations}

There are several strengths of this study. Medicaid claims data from multiple years of three states were used in this study. There are several advantages to using such administrative data. A large cohort of patients could be efficiently followed for a long period of time across a variety of providers. The study included adults with T2DM, newly-diagnosed depression and coexisting chronic physical conditions, such populations with multiple conditions are often ignored in clinical trials of depression treatment. The economic consequences of depression treatment were observed in real world settings instead of the controlled environment of clinical trials. The use of a repeated measures design allowed for studying the association between depression treatments and healthcare expenditure over time, instead of aggregating expenditures at the end of follow-up. To the best of the authors' knowledge such a design has yet not been adopted by any other study in this area. Since the association between depression treatments and healthcare expenditures adjusted for inverse probability treatment weights, selection bias due to differences in observed characteristics in the depression treatment groups could be controlled in the analyses.

However, the study has some limitations. As administrative claims data can only identify diseases through diagnosis codes, a limitation using such kind of data for studies with depression, is the potential for underestimating newly-diagnosed depression owing to undiagnosed depression and under-coding of depression. Identifying depression is one of the more difficult problems in administrative data research and perfection may not be attainable. However, the use of diagnoses codes that are recommended by HEDIS and extensively used by health plans in order to identify depression claims and the ability to identify physician/ 
psychologist diagnoses of depression offer a particularly attractive alternative to the substantial expenditure and complications associated with prospective surveys supplemented with medical record data. Additionally, T2DM and other coexisting chronic physical conditions were also identified using diagnosis codes in medical claims. Incomplete or erroneous records submitted by healthcare providers, limited clinical detail in the ICD-9-CM codes and inaccurate demographic information might limit the accuracy of administrative data. Perceived general and mental health status variables were not available due to nature of the dataset and thus could not be controlled for in the analyses. These variables may impact choice of depression treatment and expenditures. Duration of T2DM and coexisting chronic conditions may influence healthcare utilization and consequently expenditures, however as these variables were not available and were not adjusted for in regression analyses. The study included fee-for-service Medicaid beneficiaries enrolled in three states, thus results might not be generalizable to Medicaid population. Additionally, though differences in observed characteristics among depression treatment groups were controlled by using IPTW, there may be unobserved differences between the depression treatment groups which were not examined in this study. 


\section{CONCLUSION}

Among non-elderly adults with T2DM and newly diagnosed depression, compared to no depression treatment, treating depression can produce cost-savings to Medicaid. Treating depression with combined antidepressants and psychotherapy may be the best alternative to achieve consistent reduction in expenditures across all types of coexisting chronic physical conditions. For specific modalities of depression treatment (i.e. antidepressant only or psychotherapy only), cost-reductions will depend on the types of coexisting chronic physical conditions. 


\section{REFERENCES}

1. Finkelstein, E.A., et al., Prevalence and costs of major depression among elderly claimants with diabetes. Diabetes Care, 2003. 26(2): p. 415-20.

2. Nichols, L., et al., Diabetes, minor depression and health care utilization and expenditures: a retrospective database study. Cost Eff Resour Alloc, 2007. 5: p. 4.

3. Egede, L.E., D. Zheng, and K. Simpson, Comorbid depression is associated with increased health care use and expenditures in individuals with diabetes. Diabetes Care, 2002. 25(3): p. 464-70.

4. Ciechanowski, P.S., W.J. Katon, and J.E. Russo, Depression and diabetes: impact of depressive symptoms on adherence, function, and costs. Arch Intern Med, 2000. 160(21): p. 3278-85.

5. Le, T.K., S.L. Able, and M.J. Lage, Resource use among patients with diabetes, diabetic neuropathy, or diabetes with depression. Cost Eff Resour Alloc, 2006. 4: p. 18.

6. Kalsekar, I.D., et al., The effect of depression on health care utilization and costs in patients with type 2 diabetes. Manag Care Interface, 2006. 19(3): p. 39-46.

7. Simon, G.E., et al., Recovery from depression predicts lower health services costs. J Clin Psychiatry, 2006. 67(8): p. 1226-31.

8. Lustman, P.J., et al., Cognitive behavior therapy for depression in type 2 diabetes mellitus. A randomized, controlled trial. Ann Intern Med, 1998. 129(8): p. 613-21.

9. Simson, U., et al., Psychotherapy intervention to reduce depressive symptoms in patients with diabetic foot syndrome. Diabet Med, 2008. 25(2): p. 206-12.

10. Georgiades, A., et al., Changes in depressive symptoms and glycemic control in diabetes mellitus. Psychosom Med, 2007. 69(3): p. 235-41.

11. Lustman, P.J., et al., Sertraline for prevention of depression recurrence in diabetes mellitus: a randomized, double-blind, placebo-controlled trial. Arch Gen Psychiatry, 2006. 63(5): p. 521-9.

12. Lustman, P.J., et al., Fluoxetine for depression in diabetes: a randomized double-blind placebo-controlled trial. Diabetes Care, 2000. 23(5): p. 618-23.

13. Lustman, P.J., et al., Effects of nortriptyline on depression and glycemic control in diabetes: results of a double-blind, placebo-controlled trial. Psychosom Med, 1997. 59(3): p. 241-50.

14. Paile-Hyvarinen, M., K. Wahlbeck, and J.G. Eriksson, Quality of life and metabolic status in mildly depressed patients with type 2 diabetes treated with paroxetine: a doubleblind randomised placebo controlled 6-month trial. BMC Fam Pract, 2007. 8: p. 34.

15. Paile-Hyvarinen, M., K. Wahlbeck, and J.G. Eriksson, Quality of life and metabolic status in mildly depressed women with type 2 diabetes treated with paroxetine: a singleblind randomised placebo controlled trial. BMC Fam Pract, 2003. 4: p. 7.

16. Amsterdam, J.D., et al., Safety and efficacy of s-citalopram in patients with co-morbid major depression and diabetes mellitus. Neuropsychobiology, 2006. 54(4): p. 208-14.

17. Gulseren, L., et al., Comparison of fluoxetine and paroxetine in type II diabetes mellitus patients. Arch Med Res, 2005. 36(2): p. 159-65.

18. Lustman, P.J., et al., Factors influencing glycemic control in type 2 diabetes during acute- and maintenance-phase treatment of major depressive disorder with bupropion. Diabetes Care, 2007. 30(3): p. 459-66. 
19. Katon, W., et al., Cost-effectiveness and net benefit of enhanced treatment of depression for older adults with diabetes and depression. Diabetes Care, 2006. 29(2): p. 265-70.

20. Simon, G.E., et al., Cost-effectiveness of systematic depression treatment among people with diabetes mellitus. Arch Gen Psychiatry, 2007. 64(1): p. 65-72.

21. Katon, W.J., et al., Long-term effects on medical costs of improving depression outcomes in patients with depression and diabetes. Diabetes Care, 2008. 31(6): p. 1155-9.

22. Katon, W., et al., Impact of antidepressant drug adherence on comorbid medication use and resource utilization. Arch Intern Med, 2005. 165(21): p. 2497-503.

23. Chen, P.C., et al., Population-based cohort analyses of the bidirectional relationship between type 2 diabetes and depression. Diabetes Care, 2013. 36(2): p. 376-82.

24. Clarke, J.L. and D.C. Meiris, Building bridges: integrative solutions for managing complex comorbid conditions. Am J Med Qual, 2007. 22(2 Suppl): p. 5S-16S.

25. Naessens, J.M., et al., Effect of multiple chronic conditions among working-age adults. Am J Manag Care, 2011. 17(2): p. 118-22.

26. Taylor, A.W., et al., Multimorbidity - not just an older person's issue. Results from an Australian biomedical study. BMC Public Health, 2010. 10: p. 718.

27. Ward, B.W. and J.S. Schiller, Prevalence of multiple chronic conditions among US adults: estimates from the National Health Interview Survey, 2010. Prev Chronic Dis, 2013. 10: p. E65.

28. Canadian Mental Health Associatio. 2008. The Relationship between Mental Health, Mental Illness and Chronic Physical Condition. Available at : http://ontario.cmha.ca/public_policy/the-relationship-between-mental-health-mentalillness-and-chronic-physical-conditions/\#.VKLXBF4AKA.

29. de Jonge, P., et al., Prevalent and incident depression in community-dwelling elderly persons with diabetes mellitus: results from the ZARADEMP project. Diabetologia, 2006. 49(11): p. 2627-33.

30. The Kaiser Family Foundation. 2012.The Role of Medicaid for People with Diabetes. Avaiable at: http://kaiserfamilyfoundation.files.wordpress.com/2013/01/8383_d.pdf. Accessed on December 29, 2014

31. Adelmann, P.K., Mental and substance use disorders among Medicaid recipients: prevalence estimates from two national surveys. Adm Policy Ment Health, 2003. 31(2): p. 111-29.

32. Pratt, L.A. and D.J. Brody, Depression in the United States household population, 2005 2006. NCHS Data Brief, 2008(7): p. 1-8.

33. Agency for Healthcare Research and Quality. Statistical Brief \#172. 2011. Conditions With the Largest Number of Adult Hospital Readmissions by Payer, 2011. Avaiable at: http://www.hcup-us.ahrq.gov/reports/statbriefs/sb172-Conditions-ReadmissionsPayer.pdf. Accessed on December 29, 2014.

34. $\quad$ Gilmer, G., T. P., et al., Predictors of health care costs in adults with diabetes. Diabetes Care, 2005. 28(1): p. 59-64.

35. Andersen, R.M., Revisiting the behavioral model and access to medical care: does it matter? J Health Soc Behav, 1995. 36(1): p. 1-10.

36. Department of Health and Human Services. Area Health Resources Files. Avaiable at: http://ahrf.hrsa.gov/.

37. Stein, B.D., et al., Predictors of adequate depression treatment among Medicaid-enrolled youth. Soc Psychiatry Psychiatr Epidemiol, 2013. 48(5): p. 757-65. 
38. HEDIS® QUALITY MEASURES GUIDE 2013. Avaiable at: http://www.anthem.com/ca/shared/f0/s0/t0/pw_e192761.pdf?refer=provider. accessed on December 29, 2014.

39. Sambamoorthi, U., et al., Diabetes and depression care among medicaid beneficiaries. J Health Care Poor Underserved, 2006. 17(1): p. 141-61.

40. Sambamoorthi, U., et al., Depression treatment patterns among women veterans with cardiovascular conditions or diabetes. World Psychiatry, 2010. 9(3): p. 177-82.

41. Teh, C.F., et al., Predictors of adequate depression treatment among Medicaid-enrolled adults. Health Serv Res, 2010. 45(1): p. 302-15.

42. Tiwari, A., et al., Guideline-consistent antidepressant treatment patterns among veterans with diabetes and major depressive disorder. Psychiatr Serv, 2008. 59(10): p. 1139-47.

43. To, A., H. Oetter, and R.W. Lam, Treatment of depression in primary care-Part 1: Principles of acute treatment. BRITISH COLUMBIA MEDICAL JOURNAL, 2002. 44(9): p. 473-478.

44. Harpaz-Rotem, I., D. Libby, and R.A. Rosenheck, Psychotherapy use in a privately insured population of patients diagnosed with a mental disorder. Soc Psychiatry Psychiatr Epidemiol, 2012. 47(11): p. 1837-44.

45. Piette, J.D. and E.A. Kerr, The impact of comorbid chronic conditions on diabetes care. Diabetes Care, 2006. 29(3): p. 725-31.

46. Census 2000 Brief. Educational Attainment 2000. Avaiable at: http://www.census.gov/prod/2003pubs/c2kbr-24.pdf. Accessed on Dec 7,2014.

47. Census 2000 Brief. Poverty 1999. Avaiable at: http://www.census.gov/prod/2003pubs/c2kbr-19.pdf. Accessed on Dec 7,2014.

48. Schneider, R.K. and J.L. Levenson, Psychiatry essentials for primary care. 2008: ACP Press.

49. Steve Malek, M.H., Daniel Perlman., Depression treatment: Impact of treatment persistence on total healthcare costs., 2012, Milliman Research Report.

50. Petrak, F. and S. Herpertz, Treatment of depression in diabetes: an update. Curr Opin Psychiatry, 2009. 22(2): p. 211-7.

51. Rost, K., et al., The role of competing demands in the treatment provided primary care patients with major depression. Arch Fam Med, 2000. 9(2): p. 150-4.

52. Klinkman, M.S., Competing demands in psychosocial care. A model for the identification and treatment of depressive disorders in primary care. Gen Hosp Psychiatry, 1997. 19(2): p. 98-111.

53. Hollon, S.D., et al., Effect of cognitive therapy with antidepressant medications vs antidepressants alone on the rate of recovery in major depressive disorder: a randomized clinical trial. JAMA Psychiatry, 2014. 71(10): p. 1157-64.

54. de Jonghe, F., et al., Psychotherapy alone and combined with pharmacotherapy in the treatment of depression. Br J Psychiatry, 2004. 185: p. 37-45.

55. Blom, M.B., et al., Combination treatment for acute depression is superior only when psychotherapy is added to medication. Psychother Psychosom, 2007. 76(5): p. 289-97.

56. Cuijpers, P., et al., Adding psychotherapy to pharmacotherapy in the treatment of depressive disorders in adults: a meta-analysis. J Clin Psychiatry, 2009. 70(9): p. 121929.

57. Cuijpers, P., et al., Adding psychotherapy to antidepressant medication in depression and anxiety disorders: a meta-analysis. World Psychiatry, 2014. 13(1): p. 56-67. 
58. American Psychiatric Association (APA). Practice guideline for the treatment of patients with major depressive disorder. 3rd ed. Arlington (VA): American Psychiatric Association (APA); 2010 Oct. 152 p.

59. Hollon, S.D., et al., Psychotherapy and medication in the treatment of adult and geriatric depression: which monotherapy or combined treatment? J Clin Psychiatry, 2005. 66(4): p. 455-68.

60. Oestergaard, S. and C. Moldrup, Improving outcomes for patients with depression by enhancing antidepressant therapy with non-pharmacological interventions: a systematic review of reviews. Public Health, 2011. 125(6): p. 357-67.

61. Petersen, T.J., Enhancing the efficacy of antidepressants with psychotherapy. Journal of Psychopharmacology, 2006. 20(3 suppl): p. 19-28.

62. Friedman, M.A., et al., Combined psychotherapy and pharmacotherapy for the treatment of major depressive disorder. Clinical Psychology: Science and Practice, 2004. 11(1): p. 47-68.

63. Pampallona, S., et al., Combined pharmacotherapy and psychological treatment for depression: a systematic review. Archives of general psychiatry, 2004. 61(7): p. 714-719.

64. World Health Organization.Adherence to long-term therapies. Evidence for action. Avaialble at: http://www.who.int/chp/knowledge/publications/adherence full_report.pdf.

65. Balon, R., Cognitive-behavioral therapy, psychotherapy and psychosocial interventions in the medically ill. Psychother Psychosom, 2009. 78(5): p. 261-4.

66. White, C.A., Cognitive behavioral principles in managing chronic disease. West J Med, 2001. 175(5): p. 338-42.

67. Dalton, J.A. and P. Coyne, Cognitive-behavioral therapy: tailored to the individual. Nurs Clin North Am, 2003. 38(3): p. 465-76, vi.

68. Thorn, B.E. and M.C. Kuhajda, Group cognitive therapy for chronic pain. J Clin Psychol, 2006. 62(11): p. 1355-66.

69. Croghan TW, Brown JD. Integrating Mental Health Treatment Into the Patient Centered Medical Home. (Prepared by Mathematica Policy Research under Contract No.

HHSA290200900019I TO2.) AHRQ Publication No. 10-0084-EF. Rockville, MD: Agency for Healthcare Research and Quality. June 2010. .

70. National Academy of State Health Policy. Medical Home \& Patient-Centered Care. Available at : http://www.nashp.org/med-home-map. Accessed on December 31, 2014.

71. National Committee for Quality Assurance. The state of health care quality: 2007. Antidepressant Medication Management. Available at: https://www.ncqa.org/Portals/0/Publications/Resource\%20Library/SOHC/SOHC_07.pdf Accessed July 20, 2014.

72. Foa, E.B., Prolonged exposure therapy: past, present, and future. Depression and anxiety, 2011. 28(12): p. 1043-1047.

73. Fortney, J.C., et al., The association between rural residence and the use, type, and quality of depression care. J Rural Health, 2010. 26(3): p. 205-13. 
TABLES, FIGURES \& APPENDICES

Table 1: Description of the Study Population by Types of Coexisting Chronic Physical Conditions Among Medicaid Beneficiaries with Type 2 Diabetes Mellitus and Newly-diagnosed Depression Multi-state Medicaid Claims Database - 2000 - 2008

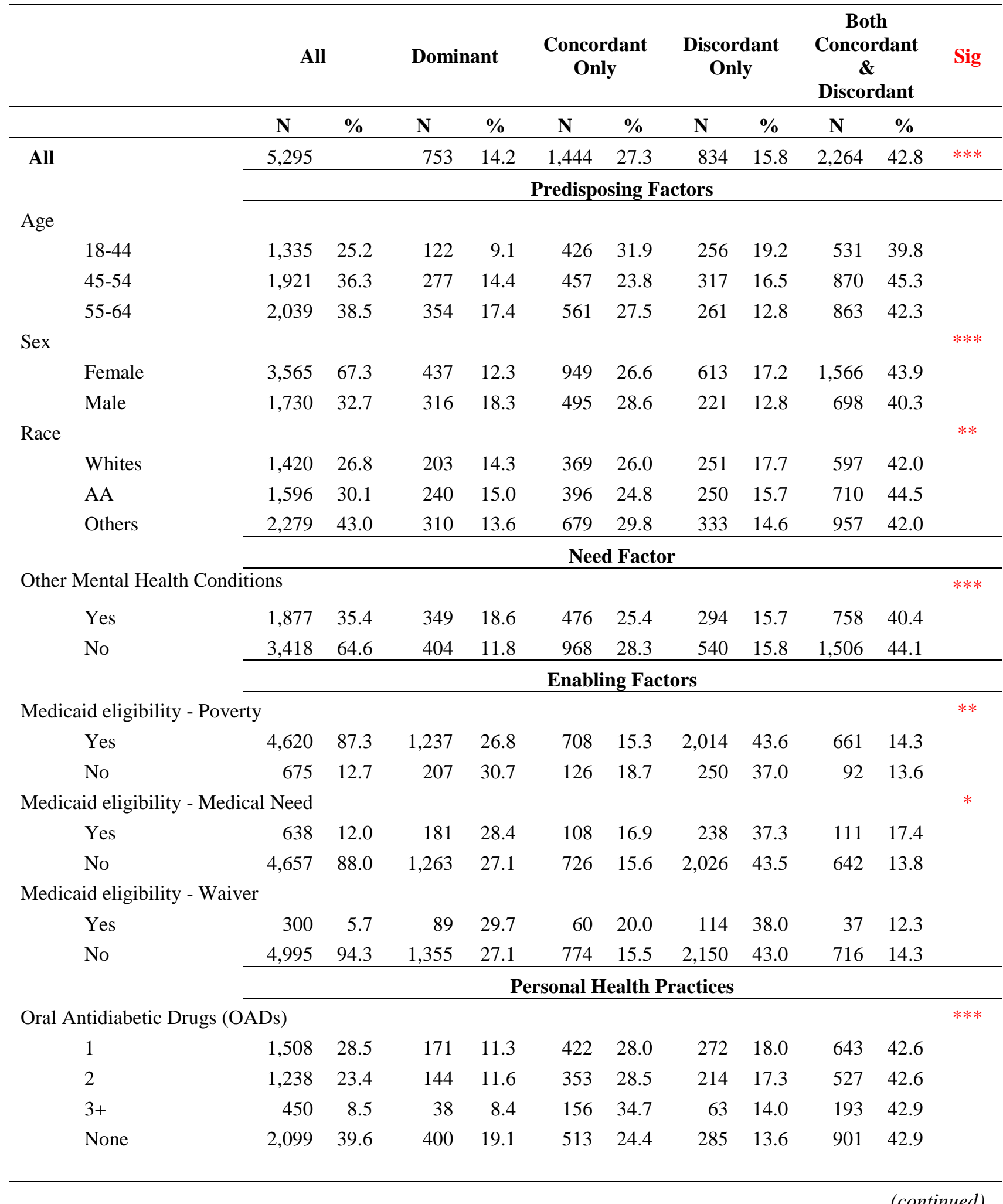


Table 1: Description of the Study Population by Types of Coexisting Chronic Physical Conditions Among Medicaid Beneficiaries with Type 2 Diabetes Mellitus and Newly-diagnosed Depression Multi-state Medicaid Claims Database - 2000 - 2008

\begin{tabular}{|c|c|c|c|c|c|c|c|c|c|c|c|}
\hline & \multicolumn{2}{|c|}{ All } & \multicolumn{2}{|c|}{ Dominant } & \multicolumn{2}{|c|}{$\begin{array}{c}\text { Concordant } \\
\text { Only }\end{array}$} & \multicolumn{2}{|c|}{$\begin{array}{l}\text { Discordant } \\
\text { Only }\end{array}$} & \multicolumn{2}{|c|}{$\begin{array}{c}\text { Both } \\
\text { Concordant } \\
\& \\
\text { Discordant }\end{array}$} & \multirow[t]{2}{*}{ Sig } \\
\hline & $\mathbf{N}$ & $\%$ & $\mathbf{N}$ & $\%$ & $\mathbf{N}$ & $\%$ & $\mathbf{N}$ & $\%$ & $\mathbf{N}$ & $\%$ & \\
\hline Insulin Use & & & & & & & & & & & $* * *$ \\
\hline Yes & 1,827 & 34.5 & 277 & 15.2 & 512 & 28.0 & 148 & 8.1 & 890 & 48.7 & \\
\hline No & 3,468 & 65.5 & 476 & 13.7 & 932 & 26.9 & 686 & 19.8 & 1,374 & 39.6 & \\
\hline Polypharmacy & & & & & & & & & & & $* * *$ \\
\hline Yes & 1,915 & 36.2 & 293 & 15.3 & 345 & 18.0 & 295 & 15.4 & 982 & 51.3 & \\
\hline No & 3,380 & 63.8 & 460 & 13.6 & 1,099 & 32.5 & 539 & 15.9 & 1,282 & 37.9 & \\
\hline Inpatient Hospitalization & & & & & & & & & & & $* * *$ \\
\hline Yes & 2,604 & 49.2 & 534 & 20.5 & 532 & 20.4 & 232 & 8.9 & 1,306 & 50.2 & \\
\hline No & 2,691 & 50.8 & 219 & 8.1 & 912 & 33.9 & 602 & 22.4 & 958 & 35.6 & \\
\hline Outpatient Visits & & & & & & & & & & & $* * *$ \\
\hline 1st Quartile & 1,392 & 26.3 & 85 & 6.1 & 588 & 42.2 & 294 & 21.1 & 425 & 30.5 & \\
\hline 2nd Quartile & 1,221 & 23.1 & 139 & 11.4 & 352 & 28.8 & 215 & 17.6 & 515 & 42.2 & \\
\hline 3rd Quartile & 1,352 & 25.5 & 227 & 16.8 & 282 & 20.9 & 180 & 13.3 & 663 & 49.0 & \\
\hline \multirow[t]{2}{*}{ 4th Quartile } & 1,330 & 25.1 & 302 & 22.7 & 222 & 16.7 & 145 & 10.9 & 661 & 49.7 & \\
\hline & \multicolumn{2}{|c|}{ Mean \pm SE } & \multicolumn{2}{|c|}{ Mean $\pm S E$} & \multicolumn{2}{|c|}{ Mean $\pm \mathrm{SE}$} & \multicolumn{2}{|c|}{$\operatorname{Mean} \pm \mathrm{SE}$} & \multicolumn{2}{|c|}{ Mean \pm SE } & \\
\hline $\begin{array}{l}\text { Number of T2DM- } \\
\text { related office visits }\end{array}$ & \multicolumn{2}{|c|}{$6.93 \pm 0.13$} & \multicolumn{2}{|c|}{$7.98 \pm 0.29$} & \multicolumn{2}{|c|}{$6.51 \pm 0.29$} & \multicolumn{2}{|c|}{$5.44 \pm 0.21$} & \multicolumn{2}{|c|}{$7.40 \pm 0.19$} & \\
\hline \multirow[t]{2}{*}{ Number of ER Visits } & \multicolumn{2}{|c|}{$1.18 \pm 0.03$} & \multicolumn{2}{|c|}{$1.65 \pm 0.11$} & \multicolumn{2}{|c|}{$0.66 \pm 0.04$} & \multicolumn{2}{|c|}{$0.78 \pm 0.05$} & \multicolumn{2}{|c|}{$1.51 \pm 0.05$} & \\
\hline & \multicolumn{10}{|c|}{ External Environment } & \\
\hline State & & & & & & & & & & & $* * *$ \\
\hline Illinois & 1,502 & 28.4 & 211 & 14.0 & 379 & 25.2 & 256 & 17.0 & 656 & 43.7 & \\
\hline New York & 2,550 & 48.2 & 402 & 15.8 & 725 & 28.4 & 405 & 15.9 & 1,018 & 39.9 & \\
\hline \multirow{2}{*}{\multicolumn{12}{|c|}{$\begin{array}{l}\text { HPSA- Mental Health } \\
\text { Care }\end{array}$}} \\
\hline & & & & & & & & & & & \\
\hline Yes & 4,405 & 83.2 & 651 & 14.8 & 1,209 & 27.4 & 679 & 15.4 & 1,866 & 42.4 & \\
\hline No & 890 & 16.8 & 102 & 11.5 & 235 & 26.4 & 155 & 17.4 & 398 & 44.7 & \\
\hline Metro & & & & & & & & & & & $* *$ \\
\hline Yes & 4,719 & 89.1 & 698 & 14.8 & 1,291 & 27.4 & 730 & 15.5 & 2,000 & 42.4 & \\
\hline No & 576 & 10.9 & 55 & 9.5 & 153 & 26.6 & 104 & 18.1 & 264 & 45.8 & \\
\hline \multicolumn{12}{|l|}{ СMHC } \\
\hline Yes & 2,590 & 48.9 & 376 & 14.5 & 702 & 27.1 & 393 & 15.2 & 1,119 & 43.2 & \\
\hline No & 2,705 & 51.1 & 377 & 13.9 & 742 & 27.4 & 441 & 16.3 & 1,145 & 42.3 & \\
\hline
\end{tabular}


Table 1: Description of the Study Population by Types of Coexisting Chronic Physical Conditions Among Medicaid Beneficiaries with Type 2 Diabetes Mellitus and Newly-diagnosed Depression Multi-state Medicaid Claims Database - 2000 - 2008

\begin{tabular}{|c|c|c|c|c|c|c|c|c|c|c|c|}
\hline & \multicolumn{2}{|c|}{ All } & \multicolumn{2}{|c|}{ Dominant } & \multicolumn{2}{|c|}{$\begin{array}{c}\text { Concordant } \\
\text { Only }\end{array}$} & \multicolumn{2}{|c|}{$\begin{array}{l}\text { Discordant } \\
\text { Only }\end{array}$} & \multicolumn{2}{|c|}{$\begin{array}{c}\text { Both } \\
\text { Concordant } \\
\& \\
\text { Discordant }\end{array}$} & \multirow[t]{2}{*}{ Sig } \\
\hline & $\mathbf{N}$ & $\%$ & $\mathbf{N}$ & $\%$ & $\mathbf{N}$ & $\%$ & $\mathbf{N}$ & $\%$ & $\mathbf{N}$ & $\%$ & \\
\hline FQHC & & & & & & & & & & & $* *$ \\
\hline Yes & 4,365 & 82.4 & 651 & 14.9 & 1,199 & 27.5 & 674 & 15.4 & 1,841 & 42.2 & \\
\hline No & 930 & 17.6 & 102 & 11.0 & 245 & 26.3 & 160 & 17.2 & 423 & 45.5 & \\
\hline Median Income & & & & & & & & & & & $* *$ \\
\hline 1st Quartile & 1,312 & 24.8 & 145 & 11.1 & 365 & 27.8 & 204 & 15.5 & 598 & 45.6 & \\
\hline 2nd Quartile & 1,415 & 26.7 & 199 & 14.1 & 378 & 26.7 & 246 & 17.4 & 592 & 41.8 & \\
\hline 3rd Quartile & 1,235 & 23.3 & 182 & 14.7 & 345 & 27.9 & 190 & 15.4 & 518 & 41.9 & \\
\hline $\begin{array}{l}\text { 4th Quartile } \\
\% \text { with GT } 4 \text { yr college } \\
\text { education }>16 \%\end{array}$ & 1,333 & 25.2 & 227 & 17.0 & 356 & 26.7 & 194 & 14.6 & 556 & 41.7 & $* *$ \\
\hline Yes & 4,399 & 83.1 & 656 & 14.9 & 1,197 & 27.2 & 704 & 16.0 & 1,842 & 41.9 & \\
\hline $\begin{array}{c}\text { No } \\
\% \text { below poverty level }\end{array}$ & 896 & 16.9 & 97 & 10.8 & 247 & 27.6 & 130 & 14.5 & 422 & 47.1 & \\
\hline GT $11.1 \%$ & & & 753 & & 1,444 & & 834 & & 2,264 & & \\
\hline Yes & 4,692 & 88.6 & 675 & 14.4 & 1,284 & 27.4 & 732 & 15.6 & 2,001 & 42.6 & \\
\hline No & 603 & 11.4 & 78 & 12.9 & 160 & 26.5 & 102 & 16.9 & 263 & 43.6 & \\
\hline & \multicolumn{2}{|c|}{ Mean \pm SE } & \multicolumn{2}{|c|}{ Mean \pm SE } & \multicolumn{2}{|c|}{ Mean \pm SE } & \multicolumn{2}{|c|}{ Mean \pm SE } & \multicolumn{2}{|c|}{ Mean $\pm \mathbf{S E}$} & \\
\hline $\begin{array}{l}\text { Density of Social } \\
\text { Workers }\end{array}$ & \multicolumn{2}{|c|}{$3.01 \pm 0.02$} & \multicolumn{2}{|c|}{$3.27 \pm 0.06$} & \multicolumn{2}{|c|}{$3.02 \pm 0.04$} & \multicolumn{2}{|c|}{$3.02 \pm 0.06$} & \multicolumn{2}{|c|}{$2.91 \pm 0.04$} & \\
\hline
\end{tabular}

_Note: Study sample comprised of adults with type 2 diabetes mellitus aged 18-64 years and with at least one coexisting dominant, concordant, discordant chronic physical condition and who were alive, not dually eligible for Medicare and continuously enrolled in fee-for-service Medicaid for at least 24 months( $N=5,295)$; includes Medicaid data from three states: Illinois, Texas, New York Asterisks $(*)$ represent significant differences in study population characteristics and coexisting chronic physical conditions categories i.e. Dominant, Concordant Only, Discordant Only and Both Concordant and Discordant, derived from chi-square statistics $* * * \mathrm{P} \leq .001 ; * * .001 \leq \mathrm{P}<.01 ; * .01 \leq \mathrm{P}<.05$.

$¥ 16 \%$ Cut off was chosen based on 2000 Census Education attainment results H1 11.1\% Cut off was chosen based on 1999 Census Poverty in 18-64 year results

HPSA: health professional shortage area; CMHC: Community Mental Health Clinic; FQHC: Federally qualified health clinic; GT: Greater Than 
Table 2: IPTW Adjusted Association Between Depression Treatment and Healthcare Expenditures, Among Medicaid Beneficiaries with Type 2 Diabetes Mellitus and Newly-diagnosed Depression Multi-state Medicaid Claims Database - 2000 - 2008 (Reference Group : No Depression Treatment)

\begin{tabular}{|c|c|c|c|c|c|c|}
\hline \multirow[b]{2}{*}{$\begin{array}{l}\text { Depression } \\
\text { Treatment }\end{array}$} & \multicolumn{3}{|c|}{ ALL Expenditures } & \multicolumn{3}{|c|}{$\begin{array}{l}\text { T2DM-Related } \\
\text { Expenditures }\end{array}$} \\
\hline & Beta & SE & Sig & Beta & SE & Sig \\
\hline Only antidepressants & -0.18 & 0.04 & $* * *$ & -0.10 & 0.05 & \\
\hline $\begin{array}{l}\text { Only psychotherapy } \\
\text { Antidepressants and }\end{array}$ & -0.25 & 0.05 & $* * *$ & -0.33 & 0.06 & $* * *$ \\
\hline Psychotherapy & -0.33 & 0.06 & $* * *$ & -0.20 & 0.07 & $* *$ \\
\hline
\end{tabular}

Model adjusted for Random effects: Random Intercept; Fixed Effects: Time in months , Depression Treatment , Predisposing (gender, age, race/ethnicity), Need Factors (other mental health conditions, Types of Co-existing Chronic Physical Conditions), Enabling Factors (Medicaid Eligibility -Poverty, Medical Need, Waiver), Personal health practices (number of ER and outpatient visits, inpatient hospitalization, number of OAD classes, insulin use and polypharmacy, total baseline healthcare expenditures), External environment characteristics: (whether county of residence had a CMHC, FQHC, was HPSA for mental health, density of social workers rural/urban status of county, median income in the county, whether percent below poverty level and percent with college education greater than national average), Other variables: Antidepressant treatment during each month of follow-up, Psychotherapy treatment during each month of follow-up and All Healthcare expenditures included Inpatient, Outpatient and Prescription Drug Related Expenditures; T2DM Related Expenditures included Inpatient and Outpatient Expenditures due to T2DM-related diagnosis. The expenditures were log transformed Asterisks indicate statistical significance and are based on mixed effects models; none i.e. no antidepressant treatment i.e. none as reference group for dependent variable. ${ }^{*} * * \mathrm{P}<.001 ; * * .001 \leq \mathrm{P}<.01 ; * .01 \leq \mathrm{P}<.05$. T2DM: Type 2 Diabetes Mellitus; depression: Major Depressive Disorder; IPTW: Inverse Probability Treatment Weights; SE: Standard Error 
Table 3: IPTW Adjusted Association Between Depression Treatment and Healthcare Expenditures Stratified by Types of Coexisting Conditions among Medicaid Beneficiaries with Type 2 Diabetes Mellitus and Newly-diagnosed Depression, Multi-state Medicaid Claims Database - 2000 - 2008, (Reference Group : No Depression Treatment)

\begin{tabular}{|c|c|c|c|c|c|c|}
\hline \multirow[b]{2}{*}{$\begin{array}{l}\text { Depression Treatment } \\
\text { Categories }\end{array}$} & \multicolumn{3}{|c|}{ ALL Expenditures } & \multicolumn{3}{|c|}{ T2DM Related Expenditures } \\
\hline & Beta & SE & Sig & Beta & SE & Sig \\
\hline & \multicolumn{6}{|c|}{ Dominant } \\
\hline Only antidepressants & -0.26 & 0.14 & & -0.26 & 0.16 & \\
\hline $\begin{array}{l}\text { Only psychotherapy } \\
\text { Antidepressants and }\end{array}$ & -0.65 & 0.12 & $* * *$ & -0.53 & 0.14 & $* * *$ \\
\hline \multirow[t]{2}{*}{ Psychotherapy } & -0.53 & 0.16 & $* *$ & -0.28 & 0.19 & \\
\hline & \multicolumn{6}{|c|}{ Concordant Only } \\
\hline Only antidepressants & -0.24 & 0.08 & $* *$ & -0.05 & 0.10 & \\
\hline $\begin{array}{l}\text { Only psychotherapy } \\
\text { Antidepressants and }\end{array}$ & -0.12 & 0.09 & & -0.21 & 0.12 & \\
\hline \multirow[t]{2}{*}{ Psychotherapy } & -0.26 & 0.11 & $*$ & -0.32 & 0.13 & $*$ \\
\hline & \multicolumn{6}{|c|}{ Discordant Only } \\
\hline Only antidepressants & -0.20 & 0.09 & $*$ & -0.09 & 0.11 & \\
\hline $\begin{array}{l}\text { Only psychotherapy } \\
\text { Antidepressants and }\end{array}$ & -0.09 & 0.13 & & -0.11 & 0.15 & \\
\hline \multirow[t]{2}{*}{ Psychotherapy } & -0.34 & 0.13 & $*$ & -0.11 & 0.16 & \\
\hline & \multicolumn{6}{|c|}{ Both Concordant \& Discordant } \\
\hline Only antidepressants & -0.11 & 0.06 & & -0.10 & 0.08 & \\
\hline $\begin{array}{l}\text { Only psychotherapy } \\
\text { Antidepressants and }\end{array}$ & -0.23 & 0.07 & $* * *$ & -0.41 & 0.10 & $* * *$ \\
\hline Psychotherapy & -0.32 & 0.08 & $* * *$ & -0.10 & 0.12 & \\
\hline
\end{tabular}

Note: Model adjusted for Random effects: Random Intercept; Fixed Effects: Adjusted for: Time in months, Depression Treatment, Predisposing (gender, age, race/ethnicity), Need Factors (other mental health conditions, Types of Co-existing Chronic Physical Conditions), Enabling Factors (Medicaid Eligibility -Poverty, Medical Need, Waiver), Personal health practices (number of ER and outpatient visits, inpatient hospitalization, number of OAD classes, insulin use and polypharmacy), External environment characteristics: (whether county of residence had a CMHC, FQHC, was HPSA for mental health, density of social workers rural/urban status of county, median income in the county, whether percent below poverty level and percent with college education greater than national average), Other variables: Antidepressant treatment at each month of follow-up, Psychotherapy treatment at each month of follow-up and Year of observation All Healthcare Expenditures included Inpatient, Outpatient and Prescription Drug Related Expenditures; T2DM Related Expenditures included Inpatient and Outpatient Expenditures due to T2DM-related diagnosis. The expenditures were log transformed

Asterisks indicate statistical significance and are based on mixed effects models; $* * * \mathrm{P}<.001 ; * * .001 \leq \mathrm{P}<.01$; $* .01 \leq \mathrm{P}<.05$.

T2DM: Type 2 Diabetes Mellitus; depression: Major Depressive Disorder; IPTW: Inverse Probability Treatment Weights; SE: Standard Error 
Figure 1: Mean IPTW adjusted Total and T2DM-Related Healthcare Expenditures during Follow-up for Medicaid Beneficiaries with T2DM and Newly-Diagnosed Depression

Total Health Care Costs

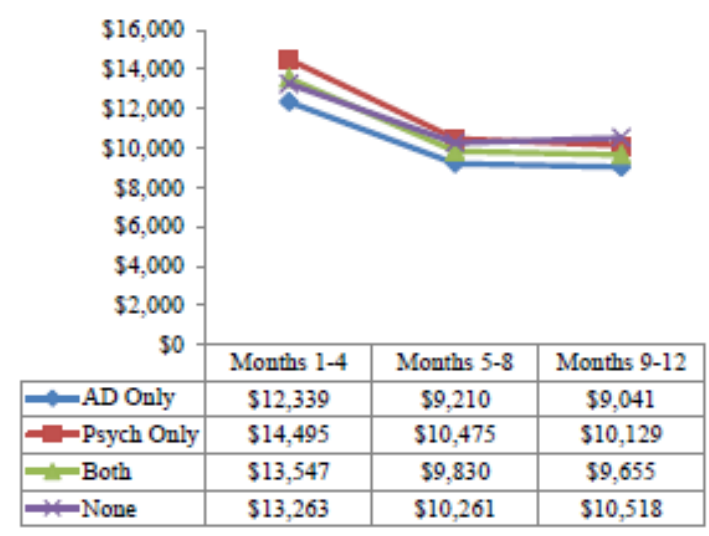

T2DM Related Health Care Costs

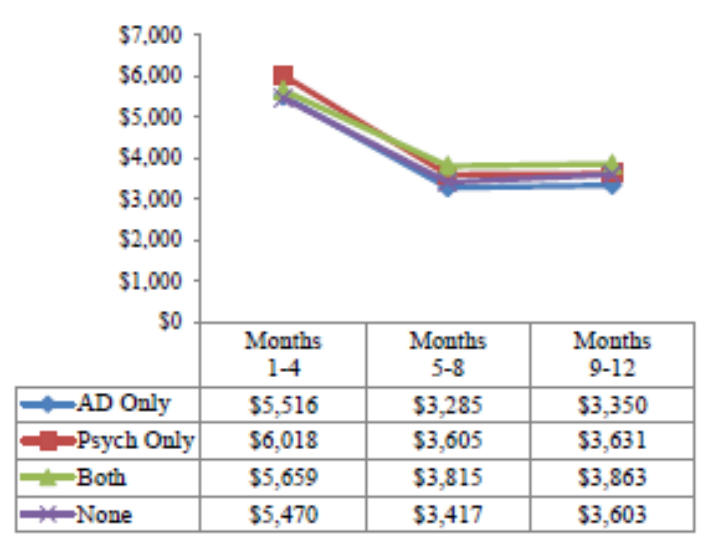


Figure 2: Mean IPTW adjusted Total and T2DM-Related Healthcare Expenditures during Follow-up for Medicaid Beneficiaries with T2DM and Newly-Diagnosed Depression: By Types of Coexisting Chronic Physical Conditions

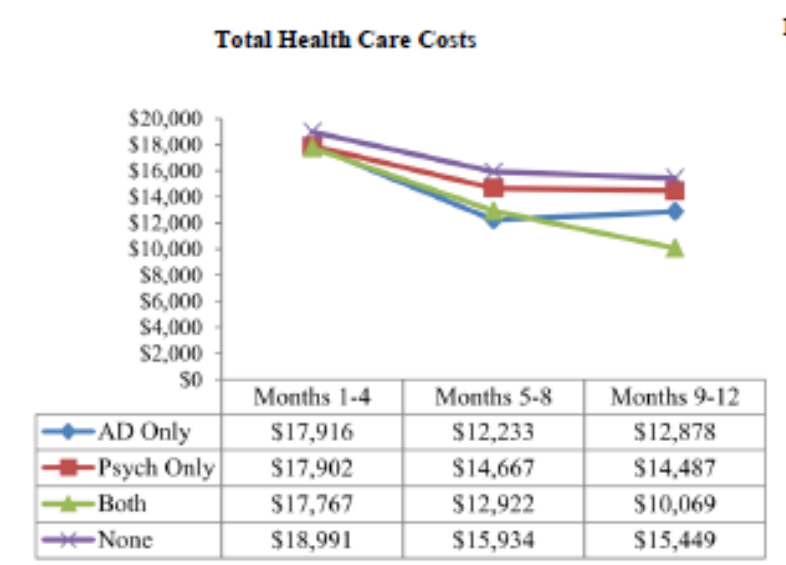

Figure 2a: Dominant Conditions

Figure 2b: Concordant Conditions Only

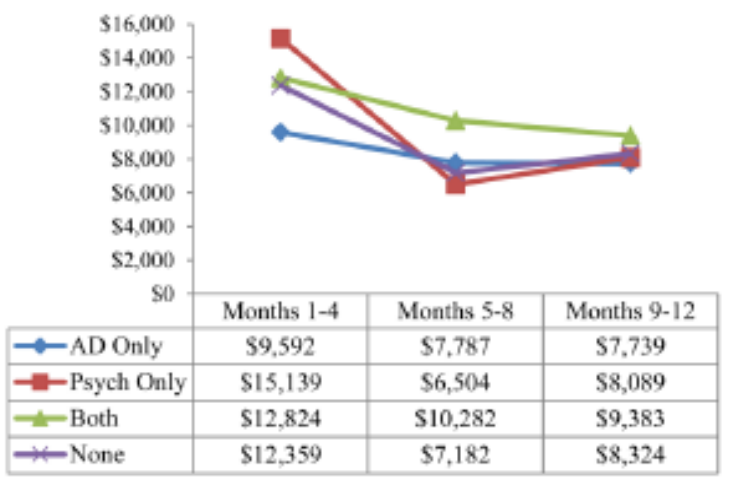

T2DM Related Health Care Costs

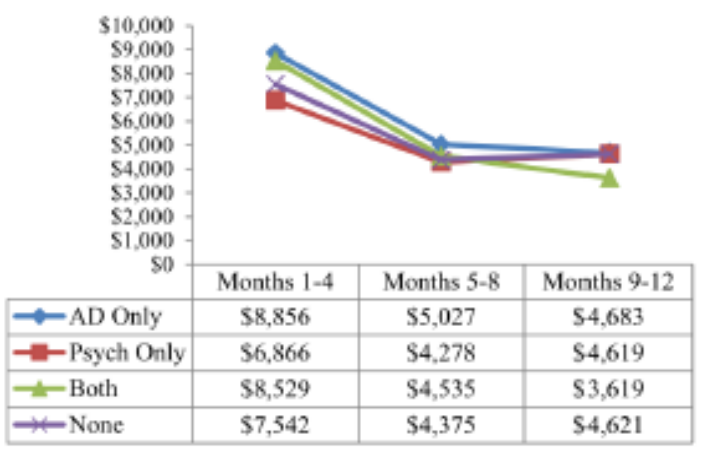

Page 150 of 173 
Total Health Care Costs

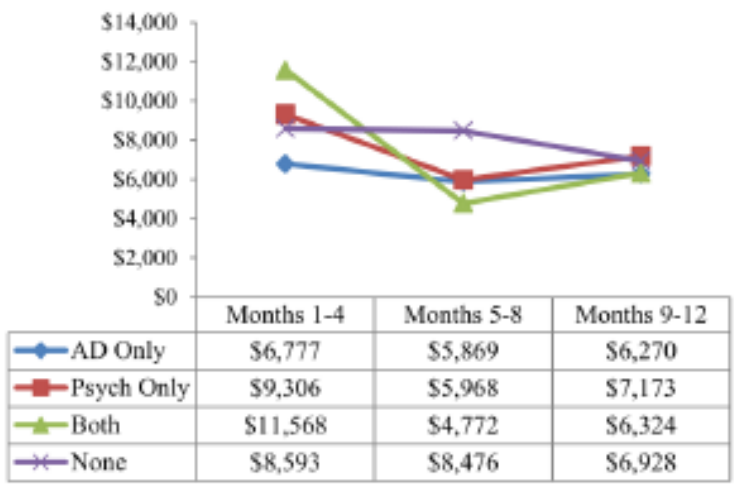

Figure 2c: Discordant

Conditions Only

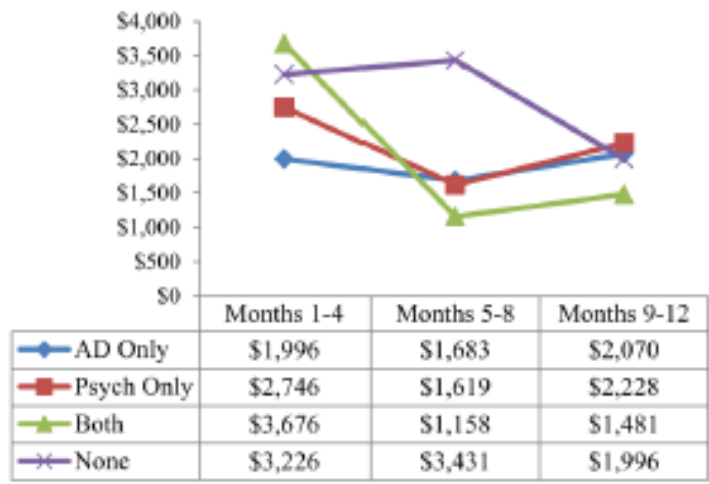

Figure 2d: Both Concordant

and Discordant Conditions

\begin{tabular}{|c|c|c|c|}
\hline \multirow{3}{*}{$\left.\begin{array}{r}\$ 16,000 \\
\$ 14,000 \\
\$ 12,000 \\
\$ 10,000 \\
\$ 8,000 \\
\$ 6,000 \\
\$ 4,000 \\
\$ 2,000 \\
\$ 0\end{array}\right]$} & \multirow{2}{*}{\multicolumn{3}{|c|}{$\mathrm{NO}^{2}$}} \\
\hline & & & \\
\hline & $\begin{array}{c}\text { Months 1- } \\
4\end{array}$ & $\begin{array}{c}\text { Months 5- } \\
8\end{array}$ & $\begin{array}{c}\text { Months 9- } \\
12\end{array}$ \\
\hline$\multimap$ AD Only & $\$ 14,138$ & $\$ 10,179$ & $\$ 9,753$ \\
\hline - -Psych Only & $\$ 14,770$ & $\$ 12,692$ & $\$ 10,278$ \\
\hline -Both & $\$ 12,718$ & $\$ 9,969$ & $\$ 10,446$ \\
\hline$\because$ None & $\$ 13,695$ & $\$ 11,211$ & $\$ 11,653$ \\
\hline
\end{tabular}

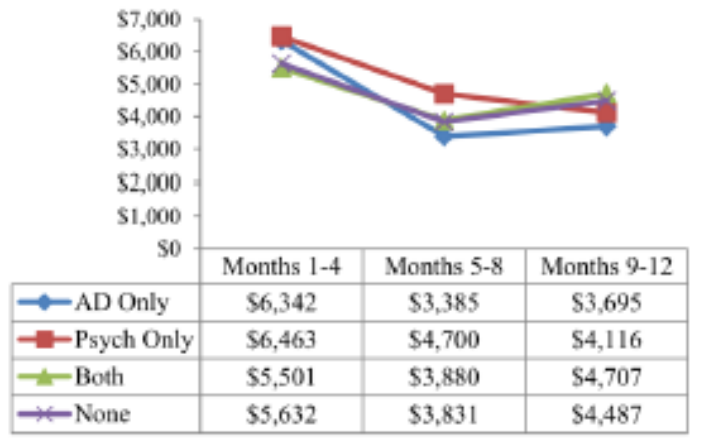

Page 151 of 173 
Appendix A : ICD-9-CM Codes for Identifying Types of Coexisting chronic physical Conditions

\begin{tabular}{|c|c|}
\hline Conditions & ICD-9-CM Codes \\
\hline $\begin{array}{l}\text { Concordant conditions } \\
\text { Coronary Artery Disease }\end{array}$ & $\begin{array}{l}410,4100,4101,4102,4103,4104,4105,4106,4107,4108,4109,411,4110, \\
4111,4118,41181,41189,412,413,4130,4131,4139,414,4140,41400,414 \\
01,41402,41403,41404,41405,4141,41410,41411,41419,4148,4149\end{array}$ \\
\hline Congestive Heart Failure & $40201,40211,40291,40401,40411,40491,428,4280,4281,4289$ \\
\hline Arrhythmia & $423,4230,4231,4232,4238,4239,42731$ \\
\hline Stroke & $\begin{array}{l}\text { 431,43301,43311,43321,43331,43381,43391,43401,43411,43491,435, } \\
\text { 4350,4351,4352,4353,4358,4359,438,4380,4381,43811,43812,4382,43 } \\
\text { 83,4384,4385,43850,4385,43852,43853,4388,43881, } \\
\text { 43882,43889,4389 }\end{array}$ \\
\hline Peripheral Vascular Disease & $\begin{array}{l}2507,4402,44020,44021,44022,44023,44024,44029,4408,4409,4422,4 \\
423,443,4430,4431,4438,44381,44389,4439,44422,44481\end{array}$ \\
\hline Peripheral Vascular Disease-gangrene & 7854 \\
\hline $\begin{array}{l}\text { Renal } \\
\text { Chronic Renal Failure } \\
\text { Chronic Pathophysiology }\end{array}$ & $\begin{array}{l}40311,40391,40412,40413,40492,40493,585,586,587, \\
2741,27410,27411,27419, \\
40310,40390,40410,40411,40490,40491, \\
581,5810,5811,5812,5813,5818,5819, \\
582,5820,5821,5822,5824,5828,58281,58289,5829,583,5830,5831,583 \\
2,5834,5836, \\
5837,5838,58381,58389,5839,5900,59000,59001,5936,5939, \\
75312,75313,75314\end{array}$ \\
\hline Diabetic Nephropathy & $2504,25040,25041,25042,25043$ \\
\hline Acute Renal Failure and Disease & $\begin{array}{l}40300,40301,40400,40401,40402,40403,40501,4533,584,5845,5846,5 \\
847,5848,5849, \\
580,5800,5804,5808,58081,58089,5809,5901,59010,59011,5902,5903, \\
5908,59080, \\
59081,59381,866,8660,86600,86601,86602,86603,8661,86610,86611, \\
86612,86613\end{array}$ \\
\hline $\begin{array}{l}\text { Retinopathy } \\
\text { (excludes advanced retinopathy, blindness) }\end{array}$ & $3620,36201,25050,25051,25052,25053$ \\
\hline Ulcer & $700,68110,68111,6827,7071,73076,73077$ \\
\hline $\begin{array}{l}\text { Other Diabetes Related Complications } \\
\text { Uncontrolled Diabetes } \\
\text { Short Term Diabetes } \\
\text { Discordant Conditions }\end{array}$ & $\begin{array}{l}25002,25003,25010,25011,25012,25013,25020,25021,25022,25023,25 \\
030,25031, \\
25032,25033\end{array}$ \\
\hline $\begin{array}{l}\text { Gastro Intestinal Tract Related Disorders: } \\
\text { GERD/Esophagitis } \\
\text { Peptic ulcers } \\
\text { Inflammatory Bowel Disease } \\
\text { Diverticulitis } \\
\text { Gall Bladder disease and stone } \\
\text { Viral hepatitis } \\
\text { Chronic Obstructive Pulmonary Disease }\end{array}$ & $\begin{array}{l}5301,5302,5303,53081,531,532,533,534,555,556,56211,56213,574 \\
575,576,070\end{array}$ \\
\hline Gout & 274,712 \\
\hline Low back pain & $\begin{array}{l}\text { 71905,71915,71925,71935,71945,71955,71965,71975,71985,71995,72 } \\
65,73314,73315,73342,820 \\
720,7213,72142,72210,72252,72273,72283, \\
72293,72402,7242,7243,7244,7245,7246,7247,7248,7249\end{array}$ \\
\hline
\end{tabular}

\section{Page 152 of 173}


Appendix A : ICD-9-CM Codes for Identifying Types of Coexisting chronic physical Conditions

\begin{tabular}{|c|c|}
\hline Conditions & ICD-9-CM Codes \\
\hline Osteoarthritis & 715 \\
\hline Other Arthritis & 716 \\
\hline Rheumatoid arthritis & 714 \\
\hline Connective tissue rheumatological disease & $7100,7101,7104,725$ \\
\hline Blindness Single Eye & $3696,3697,3698,3699$ \\
\hline \multicolumn{2}{|l|}{ Dominant Conditions } \\
\hline End Stage Renal Disease & E8791,V51,V56,V560,V5631,V5632,V568 \\
\hline End Stage Liver Disease & $5722,5723,5724,5728,4560,4561,4562,45620,45621,571$ \\
\hline Blindness Both Eyes/ Advanced Retinopathy & $36202,3690,3691,3692,3693,3694$ \\
\hline Cancer & $\begin{array}{l}140,141,142,143,144,145,146,147,148,149,150,151,152,153,154,155,1 \\
56,157,158,159,160,161,162,163,164,165,166,167,168,169,170,171,17 \\
2,174,175,176,177,178,179,180,181,182,183,184,186,187,188,189,190 \\
, 191,192,193,194,195,196,197,198,199,200,201,202,203,204,205,206, \\
207,208\end{array}$ \\
\hline Pre-dementia Cognitive Impairment & $294,2941,29283,2949,33183,78093,438,3330,3334,3315$ \\
\hline Dementia and Related Conditions & $\begin{array}{l}2900,29010,29011,29012,29013,2902,29021,2903,29040,29041,29042 \\
\text {,29043,2912, } \\
29410,29411,2948,3310,3311,3312,3317,33182,33189,3319,3320,046 \\
1,0463,0941, \\
29282,3109\end{array}$ \\
\hline Multiple Sclerosis & 340 \\
\hline Hemiplegia Hemiparesis and Paraplegia & 342,3441 \\
\hline Parkinson's Disease & 332 \\
\hline Muscular dystrophy & 359 \\
\hline Spinal cord injury & $\begin{array}{l}80600,80601,80602,80603,80604,80605,80606,80607,80608,80609,80 \\
61,9520, \\
34400,34401,34402,34403,34404,34409\end{array}$ \\
\hline Epilepsy & 345 \\
\hline Gastropareis & 5363 \\
\hline AIDS & 042 \\
\hline
\end{tabular}

\section{Page 153 of 173}




\section{Appendix B: Secondary Analysis}

Adequate depression Treatment during the acute phase: Among those who received depression treatment either with antidepressant or psychotherapy, adequate depression treatment was classified as: (1) Received Adequate Treatment with Antidepressants or Psychotherapy: Based on HEDIS definition adequate antidepressant treatment was defined as receiving 84 or more days of prescription for any antidepressant drug during the first 120 days following the index date of depression diagnosis $[1,2]$. Those who received 8 or more sessions of psychotherapy office visits (the minimum length of evidence based psychotherapy treatment for depression and anxiety disorders) $[3,4]$ during the 120 days of acute phase treatment were considered as having Adequate treatment with psychotherapy. Those who received adequate treatment either with antidepressants or psychotherapy were considered as having received adequate treatment during the acute phase (2) Not Adequate depression Treatment: Individuals who did not receive adequate treatment either with antidepressants or psychotherapy were considered as not being adequately treated.

\section{Unadjusted Association between Types of Coexisting Chronic Physical Conditions and Adequate Depression} Treatment: Over-all, 56.78\% ( $\mathrm{N}=3,007)$ of the study population received depression treatment with antidepressants and/or psychotherapy. Of these individuals $32.46 \%$ received adequate depression treatment; $32.2 \%$ of those with dominant conditions ( $\mathrm{N}=451), 34.1 \%$ of those with concordant conditions $(\mathrm{N}=804), 31.7 \%$ of those discordant conditions ( $\mathrm{N}=479)$ and $31.8 \%$ of those with both discordant and concordant conditions $(\mathrm{N}=1,273)$ received adequate depression treatment. Results of chi-square analysis showed that the association between types of coexisting chronic physical conditions and adequate depression treatment was not statistically significant. These results are not presented in tabular form

Adjusted Association between Types of Adequate Depression Treatment and Expenditures: The linear mixed model regression analysis results showed that adequate depression treatment was not associated with total healthcare expenditures. As compared to bot adequate depression treatment, adequate depression treatment was not significantly associated with log transformed T2DM-related healthcare expenditures (beta coefficient: 0.27 , standard error: 0.07; p-value <0.0001) Model 1, which did not control for interaction between adequate depression treatment and types of coexisting chronic physical conditions; however the significance disappeared after controlling for the interaction term suggesting that the association between adequate depression treatment and T2DM-related expenditures differed by types of coexisting chronic physical conditions.

\section{Adjusted Association between Types of Adequate Depression Treatment And Expenditures: By Coexisting} Chronic Physical Conditions: When separate linear mixed model regressions where conducted among each subgroup of types of coexisting chronic physical conditions, no statistically significant association was observed between adequate depression treatment and log transformed total healthcare expenditures.

\section{REFERENCES}

1. Teh, C.F., et al., Predictors of adequate depression treatment among Medicaid-enrolled adults. Health Serv Res, 2010. 45(1): p. 302-15.

2. National Committee for Quality Assurance. The state of health care quality: 2007. Antidepressant Medication Management. Available at: https://www.ncqa.org/Portals/0/Publications/Resource\%20Library/SOHC/SOHC_07.pdf Accessed July 20, 2014.

3. Foa, E.B., Prolonged exposure therapy: past, present, and future. Depression and anxiety, 2011. 28(12): p. 1043-1047.

4. Fortney, J.C., et al., The association between rural residence and the use, type, and quality of depression care. J Rural Health, 2010. 26(3): p. 205-13. 
Appendix C: IPTW Adjusted Linear Mixed Model Regression on Healthcare Expenditures, Among Medicaid Beneficiaries with Type 2 Diabetes Mellitus and Newly-diagnosed Depression Multi-state Medicaid Claims Database - 2000 - 2008

\begin{tabular}{|c|c|c|c|c|c|c|}
\hline & \multicolumn{3}{|c|}{$\begin{array}{l}\text { Total Healthcare } \\
\text { Expenditure }\end{array}$} & \multicolumn{3}{|c|}{$\begin{array}{l}\text { T2DM-Related Healthcare } \\
\text { Expenditure }\end{array}$} \\
\hline & Beta & $\begin{array}{l}\text { Standard } \\
\text { Error }\end{array}$ & Sig & Beta & $\begin{array}{l}\text { Standard } \\
\text { Error }\end{array}$ & Sig \\
\hline Time in Months & -0.06 & 0.00 & **** & -0.08 & 0.00 & $* * *$ \\
\hline \multicolumn{7}{|l|}{ Depression Treatment } \\
\hline Only antidepressants & -0.18 & 0.04 & $* * *$ & -0.10 & 0.05 & \\
\hline Only psychotherapy & -0.25 & 0.05 & $* * *$ & -0.33 & 0.06 & $* * *$ \\
\hline Both Antidepressants and Psychotherapy & -0.33 & 0.06 & $* * *$ & -0.20 & 0.07 & $* *$ \\
\hline No Treatment & & & & 0.00 & & \\
\hline \multicolumn{7}{|l|}{ Each Month Antidepressant Treatment } \\
\hline Yes & 0.77 & 0.02 & $* * *$ & 0.31 & 0.04 & $* * *$ \\
\hline No & 0.00 & & & 0.00 & & \\
\hline \multicolumn{7}{|l|}{ Each Month Psychotherapy Treatment } \\
\hline Yes & 0.80 & 0.03 & $* * *$ & 0.71 & 0.05 & $* * *$ \\
\hline No & 0.00 & & & 0.00 & & \\
\hline \multicolumn{7}{|c|}{ Types of Coexisting Chronic Physical Conditions } \\
\hline Concordant Only & -0.01 & 0.05 & & 0.17 & 0.07 & * \\
\hline Both Concordant \& Discordant & 0.01 & 0.05 & & 0.23 & 0.06 & $* * *$ \\
\hline Dominant & -0.11 & 0.06 & & 0.03 & 0.08 & \\
\hline Discordant Only & 0.00 & & & 0.00 & & \\
\hline \multicolumn{7}{|l|}{ Age } \\
\hline 18-44 years & 0.18 & 0.04 & $* * *$ & 0.07 & 0.05 & \\
\hline $45-54$ years & 0.13 & 0.04 & $* *$ & 0.12 & 0.06 & * \\
\hline 55-64 years & 0.00 & & & 0.00 & & \\
\hline \multicolumn{7}{|l|}{ Female } \\
\hline Female & -0.04 & 0.04 & & -0.06 & 0.05 & \\
\hline Male & 0.00 & & & 0.00 & & \\
\hline \multicolumn{7}{|l|}{ Race } \\
\hline White & 0.03 & 0.05 & & -0.10 & 0.06 & \\
\hline African American & 0.01 & 0.04 & & 0.04 & 0.05 & \\
\hline Others & 0.00 & & & 0.00 & & \\
\hline \multicolumn{7}{|l|}{ State } \\
\hline Illinois & -0.05 & 0.06 & & -0.18 & 0.08 & $*$ \\
\hline New York & 0.26 & 0.07 & $* * *$ & 0.31 & 0.09 & $* * *$ \\
\hline Texas & 0.00 & & & 0.00 & & \\
\hline \multicolumn{7}{|l|}{ Other Mental Health Conditions } \\
\hline Yes & 0.19 & 0.04 & $* * *$ & 0.09 & 0.05 & * \\
\hline No & 0.00 & & & 0.00 & & \\
\hline
\end{tabular}


Appendix C: IPTW Adjusted Linear Mixed Model Regression on Healthcare Expenditures, Among Medicaid Beneficiaries with Type 2 Diabetes Mellitus and Newly-diagnosed Depression Multi-state Medicaid Claims Database - 2000 - 2008

\begin{tabular}{|c|c|c|c|c|c|c|}
\hline & \multicolumn{3}{|c|}{$\begin{array}{l}\text { Total Healthcare } \\
\text { Expenditure }\end{array}$} & \multicolumn{3}{|c|}{$\begin{array}{c}\text { T2DM-Related Healthcare } \\
\text { Expenditure }\end{array}$} \\
\hline & Beta & $\begin{array}{l}\text { Standard } \\
\text { Error }\end{array}$ & Sig & Beta & $\begin{array}{l}\text { Standard } \\
\text { Error }\end{array}$ & Sig \\
\hline \multicolumn{7}{|l|}{ Medicaid Eligibility-Poverty } \\
\hline Yes & -0.16 & 0.07 & $*$ & -0.09 & 0.09 & \\
\hline No & 0.00 & & & 0.00 & & \\
\hline \multicolumn{7}{|l|}{ Medicaid Eligibility-Medical Needs } \\
\hline Yes & 0.31 & 0.06 & $* * *$ & 0.06 & 0.08 & \\
\hline No & 0.00 & & & 0.00 & & \\
\hline \multicolumn{7}{|l|}{ Medicaid Eligibility-Waiver } \\
\hline Yes & -0.05 & 0.09 & & -0.05 & 0.11 & \\
\hline No & 0.00 & & & 0.00 & & \\
\hline \multicolumn{7}{|l|}{ Oral Antidiabetic Drugs (OADs) } \\
\hline $1 \mathrm{OAD}$ & 0.08 & 0.04 & & 0.06 & 0.06 & \\
\hline $2 \mathrm{OAD}$ & 0.18 & 0.06 & $* *$ & 0.14 & 0.08 & \\
\hline $3 \mathrm{OAD}$ & -0.20 & 0.04 & $* * *$ & -0.12 & 0.05 & $*$ \\
\hline No OAD & 0.00 & & & 0.00 & & \\
\hline \multicolumn{7}{|l|}{ Insulin Use } \\
\hline Yes & 0.27 & 0.04 & $* * *$ & 0.60 & 0.05 & $* * *$ \\
\hline No & 0.00 & & & 0.00 & & \\
\hline \multicolumn{7}{|l|}{ Polypharmacy } \\
\hline Yes & 0.47 & 0.04 & $* * *$ & -0.03 & 0.05 & \\
\hline No & 0.00 & & & 0.00 & & \\
\hline \multicolumn{7}{|l|}{ Inpatient Hospitalization } \\
\hline Yes & -0.10 & 0.04 & $*$ & 0.23 & 0.05 & $* * *$ \\
\hline No & 0.00 & & & 0.00 & & \\
\hline \multicolumn{7}{|l|}{ Outpatient Visits } \\
\hline 1 st Quartile & 0.47 & 0.05 & $* * *$ & 0.11 & 0.06 & \\
\hline 2nd Quartile & 0.85 & 0.05 & $* * *$ & 0.31 & 0.06 & $* * *$ \\
\hline 3rd Quartile & 1.20 & 0.06 & $* * *$ & 0.32 & 0.07 & $* * *$ \\
\hline 4th Quartile & 0.00 & & & 0.00 & & \\
\hline Number of T2DM Related Office Visits & 0.00 & 0.00 & & 0.06 & 0.00 & $* * *$ \\
\hline Number of ER Visits & 0.02 & 0.01 & $*$ & 0.09 & 0.01 & $* * *$ \\
\hline \multicolumn{7}{|l|}{ HPSA-Mental Health } \\
\hline Yes & 0.01 & 0.06 & & 0.22 & 0.07 & $* *$ \\
\hline No & 0.00 & & & 0.00 & & \\
\hline
\end{tabular}


Appendix C: IPTW Adjusted Linear Mixed Model Regression on Healthcare Expenditures, Among Medicaid Beneficiaries with Type 2 Diabetes Mellitus and Newly-diagnosed Depression Multi-state Medicaid Claims Database - 2000 - 2008

\begin{tabular}{|c|c|c|c|c|c|c|}
\hline & \multicolumn{3}{|c|}{$\begin{array}{l}\text { Total Healthcare } \\
\text { Expenditure }\end{array}$} & \multicolumn{3}{|c|}{$\begin{array}{c}\text { T2DM-Related Healthcare } \\
\text { Expenditure }\end{array}$} \\
\hline & Beta & $\begin{array}{l}\text { Standard } \\
\text { Error }\end{array}$ & Sig & Beta & $\begin{array}{l}\text { Standard } \\
\text { Error }\end{array}$ & Sig \\
\hline \multicolumn{7}{|l|}{ Metro } \\
\hline Yes & -0.05 & 0.08 & & -0.03 & 0.10 & \\
\hline No & 0.00 & & & 0.00 & & \\
\hline \multicolumn{7}{|l|}{ СМHC } \\
\hline Yes & -0.07 & 0.04 & & 0.08 & 0.06 & \\
\hline No & 0.00 & & & 0.00 & & \\
\hline FQHC & & & & & & $* * *$ \\
\hline Yes & 0.06 & 0.06 & & 0.27 & 0.07 & \\
\hline No & 0.00 & & & 0.00 & & \\
\hline Density Social Workers & 0.03 & 0.02 & & 0.06 & 0.03 & * \\
\hline \multicolumn{7}{|l|}{ Median Household Income } \\
\hline 1st Quartile & -0.21 & 0.08 & $*$ & 0.05 & 0.11 & \\
\hline 2nd Quartile & -0.18 & 0.06 & $* *$ & -0.17 & 0.08 & $*$ \\
\hline 3rd Quartile & -0.11 & 0.06 & & -0.06 & 0.08 & \\
\hline 4th Quartile & 0.00 & & & 0.00 & & \\
\hline \multicolumn{7}{|l|}{$\%$ with GT $4 y r$ college education $>16 \%$} \\
\hline Yes & -0.13 & 0.06 & $*$ & -0.40 & 0.08 & $* * *$ \\
\hline No & 0.00 & & & 0.00 & & \\
\hline \multicolumn{7}{|l|}{$\%$ below poverty level GT $11.1 \%$} \\
\hline Yes & -0.09 & 0.07 & & -0.28 & 0.10 & $* *$ \\
\hline No & 0.00 & & & 0.00 & & \\
\hline Baseline total healthcare expenditure & 0.00 & 0.00 & $* * *$ & 0.00 & 0.00 & $* * *$ \\
\hline \multicolumn{7}{|l|}{ Year } \\
\hline $2000-2002$ & 0.16 & 0.08 & $*$ & 0.53 & 0.10 & $* * *$ \\
\hline $2001-2003$ & 0.25 & 0.08 & $* *$ & 0.61 & 0.10 & $* * *$ \\
\hline $2002-2004$ & 0.23 & 0.08 & $* *$ & 0.57 & 0.10 & $* * *$ \\
\hline $2003-2005$ & 0.16 & 0.07 & $*$ & 0.45 & 0.10 & $* * *$ \\
\hline 2004-2006 & 0.14 & 0.07 & $*$ & 0.25 & 0.09 & $* *$ \\
\hline $2005-2007$ & 0.18 & 0.07 & $* *$ & 0.26 & 0.09 & $* *$ \\
\hline $2006-2008$ & 0.00 & & & 0.00 & & \\
\hline
\end{tabular}

Note: Asterisks indicate statistical significance and are based on mixed effects models; none i.e. no antidepressant treatment i.e. none as reference group for dependent variable. ${ }^{*} * \mathrm{P}<.001 ; * * .001 \leq \mathrm{P}<.01 ; * .01 \leq \mathrm{P}<.05$.

${ }^{\mathrm{I}}$ Both: Both Antidepressants and Psychotherapy; ${ }^{\mathrm{H}}$ None: Neither antidepressants nor Psychotherapy; SE: Standard Error 
CHAPTER 5

Page 158 of 173 


\section{CHAPTER 5: SUMMARY AND CONCLUSIONS}

\section{Study Summary}

The relationship between types of physical conditions and risk of depression is poorly understood. It is plausible that adults with T2DM and coexisting chronic physical conditions with similar disease management strategies may be at a lower risk for developing depression compared to those with coexisting chronic physical conditions that may require contradicting clinical and self-management strategies. As a majority of individuals with T2DM live with multiple other coexisting physical conditions, it is essential to examine whether individuals with particular types of coexisting physical conditions are at higher risk of developing depression as compared to other groups. In aim 1 of this dissertation, the association of risk of newlydiagnosed depression with types of coexisting physical conditions among adults with T2DM was examined.

Types of coexisting physical conditions were identified based on forty four different physical conditions commonly co-occurring among individuals with T2DM. These conditions were identified as dominant (life threatening severe conditions whose management takes priority over all other coexisting conditions) conditions, concordant (conditions with management or pathophysiology similar to that of T2DM) and discordant (conditions with management or pathophysiology different from that of T2DM). The types of coexisting physical conditions were classified following a hierarchy. Dominant conditions were first identified. Among those without dominant conditions concordant and discordant conditions were grouped. The final classification consisted of: 1) dominant 2) concordant only 3) discordant only and 4) both concordant and discordant conditions.

Depression treatment among adults with T2DM is challenging. Randomized clinical trials have proven that treating depressions helps in relieving depressive symptoms; however, the 
effect of treating depression on T2DM outcomes is not clear [1-5]. In addition, antidepressants (one of the main modalities of depression treatment) have been shown to increase the risks of cardiovascular morbidity and mortality [6,7]. As many adults with T2DM also have coexisting cardiovascular diseases, physicians may be cautious in prescribing antidepressant treatment for adults with T2DM and coexisting chronic physical conditions such as hypertension and heart disease. While there have been studies on depression treatment using collaborative care models, there real-world practices of managing depression among adults with T2DM is not known. Therefore, in aim 2 of this dissertation the association between types of coexisting chronic physical conditions and depression treatment among adults with T2DM and newly-diagnosed depression was analyzed.

The economic benefits of treating depression among adults with T2DM have not been studied widely. While randomized controlled trials have demonstrated that depression treatment delivered in collaborative care settings reduced expenditures among individuals with T2DM and coexisting depression, studies not specific to T2DM population and conducted using real world observational data have reported inconsistent findings. Therefore, it remains to be established whether depression treatment with antidepressants and psychotherapy, alone or in combination, are associated with reductions in healthcare expenditures, among individuals with T2DM. Therefore, in aim 3 of this study, whether the relationship between depression treatment and total and type 2 diabetes mellitus (T2DM) -related healthcare care expenditures vary by types of coexisting chronic physical conditions among non-elderly adult Medicaid beneficiaries with T2DM and newly-diagnosed depression was examined. 


\section{Summary of Findings}

One in ten Medicaid beneficiaries with T2DM developed depression. The risk of developing depression among those with T2DM varied by types of coexisting conditions; Medicaid beneficiaries with T2DM and dominant conditions and those with both concordant and discordant conditions were at higher risk of developing newly-diagnosed depression. Four in ten individuals in the study population did not receive any treatment for newly-diagnosed depression. Individuals with coexisting chronic physical conditions with dominant conditions were less likely to receive depression treatment with antidepressants. However, among nonelderly adults with T2DM and newly-diagnosed depression, compared to no depression treatment, treating depression produced cost-savings to Medicaid. The results indicated that treating depression with combined antidepressants and psychotherapy may be the best alternative to achieve consistent reduction in expenditures across all types of coexisting chronic physical conditions. For specific modalities of depression treatment cost-reductions would depend on the types of coexisting chronic physical conditions.

\section{Clinical and policy implications of the findings}

The study findings suggest that to reduce this increased risk of newly-diagnosed depression, those with dominant conditions and combinations of concordant and discordant conditions need to be under constant surveillance. However, in order for surveillance to be effective, in case such individuals get diagnosed with depression, appropriate treatment and follow-up must be provided. Future research may examine specific dominant conditions and combinations of concordant and discordant conditions that may result in high risk of newly diagnosed depression among adults with T2DM.

The low depression treatment rates observed in this study population may suggest that physicians are prioritizing treatment of other coexisting conditions in this study population. 
Individuals with both physical (i.e. T2DM, coexisting chronic physical conditions) and mental health (i.e. depression) often have complex health care needs which may not be fully met in traditionally short (often channeled in to 15 minute time slots) primary care visits [8]. Integrating physical and mental healthcare with carefully selected teams having backgrounds in both physical and mental health care delivery may improve quality of depression treatment among such individuals The novel healthcare delivery models such as Accountable Care Organizations (ACO) and Patient Centered Medical Home (PCMH) created under the 2010 Patient Protection and Affordable Care Act, provide options for such integrated care models.

The study results indicated that depression treatment is associated with reduced healthcare expenditures; however the association varies by types of coexisting chronic physical conditions. Such findings have important implications with respect to new payment models for healthcare services such as "bundled payment" and "expenditure benchmarking". Under bundled payment systems, where only a certain amount of prospective payment is made for episodes of care provided for a condition, while treating depression individuals with T2DM and other coexisting conditions, physicians may want to refrain from initiating depression treatment types which were no better associated with reduced healthcare expenditures as compared to not providing depression treatment all. While setting expenditure benchmarks for individuals with T2DM, newly-diagnosed depression and other coexisting chronic physical conditions, one will need to risk adjust for type of coexisting conditions and types of depression treatment categories.

\section{Unique contribution of the study}

(1) Filled a critical knowledge gap: This study provided evidence on the extent to which different types of depression treatments are economically beneficial, in the presence of T2DM and multiple coexisting chronic physical conditions (2) Use of Medicaid Claims data, feasibility 
of identifying treatments, longitudinal design: The use of administrative claims data with dates of services made it possible to follow a cohort of patients with T2DM over time and to capture the healthcare experiences of Medicaid beneficiaries across a variety of providers. The study used a comprehensive list of independent variables by merging Medicaid claims data with the countylevel data from the AHRF. (3) Use of framework to identify coexisting chronic physical conditions: A pragmatic practice-based theoretical framework was used to identify the numerous coexisting chronic physical conditions into manageable and clinically meaningful categories. (4) Real-world experience: This study was more in line with real world experiences of the patients, the effectiveness of depression treatments were examined among individuals with multiple coexisting chronic physical conditions, a population often not included in clinical trials [9].

\section{Limitations}

As administrative claims data can only identify diseases through diagnosis codes, there is the potential for underestimating depression prevalence or incidence because of under-coding of depression. Identifying depression is one of the more difficult problems in administrative data research and perfection may not be attainable. However, claims data contain physician/ psychologist diagnoses of depression and offer a particularly attractive alternative to the substantial cost and complications associated with prospective surveys supplemented with medical record data. Additionally, this study included only filled prescriptions and it is not known whether the antidepressants were actually used. This study did not include alternative forms of treatment for depression. The study included fee-for-service Medicaid beneficiaries enrolled three states, thus results might not be generalizable to other populations. 


\section{REFERENCES}

1. Lustman, P.J., et al., Sertraline for prevention of depression recurrence in diabetes mellitus: a randomized, double-blind, placebo-controlled trial. Arch Gen Psychiatry, 2006. 63(5): p. 521-9.

2. Lustman, P.J., et al., Fluoxetine for depression in diabetes: a randomized double-blind placebo-controlled trial. Diabetes Care, 2000. 23(5): p. 618-23.

3. Lustman, P.J., et al., Effects of nortriptyline on depression and glycemic control in diabetes: results of a double-blind, placebo-controlled trial. Psychosom Med, 1997. 59(3): p. 241-50.

4. Lustman, P.J., et al., Cognitive behavior therapy for depression in type 2 diabetes mellitus. A randomized, controlled trial. Ann Intern Med, 1998. 129(8): p. 613-21.

5. Paile-Hyvarinen, M., K. Wahlbeck, and J.G. Eriksson, Quality of life and metabolic status in mildly depressed patients with type 2 diabetes treated with paroxetine: a doubleblind randomised placebo controlled 6-month trial. BMC Fam Pract, 2007. 8: p. 34.

6. Glassman, A.H., et al., Sertraline treatment of major depression in patients with acute MI or unstable angina. JAMA, 2002. 288(6): p. 701-9.

7. Carney, R.M., et al., Depression and late mortality after myocardial infarction in the Enhancing Recovery in Coronary Heart Disease (ENRICHD) study. Psychosom Med, 2004. 66(4): p. 466-74.

8. Margolius, D. and T. Bodenheimer, Transforming primary care: from past practice to the practice of the future. Health Aff (Millwood), 2010. 29(5): p. 779-84.

9. Van Spall, H.G., et al., Eligibility criteria of randomized controlled trials published in high-impact general medical journals: a systematic sampling review. JAMA, 2007.

297(11): p. 1233-40. 